\title{
THERMAL MODELING AND VALIDATION TESTING OF A MINIATURE XENON ION THRUSTER
}

\author{
A Thesis \\ Presented to \\ the Faculty of California Polytechnic State University \\ San Luis Obispo
}

\author{
In Partial Fulfillment \\ of the Requirements for the Degree \\ Master of Science in Aerospace Engineering
}

by

Samuel R. Parker

December 2013 
(C) 2013

Samuel R. Parker

ALL RIGHTS RESERVED 


\section{COMMITTEE MEMBERSHIP}

TITLE:

Thermal Modeling and Validation Testing of a Miniature Xenon Ion Thruster

AUTHOR: Samuel R. Parker

DATE SUBMITTED: $\quad$ December 2013

COMMITTEE CHAIR: Kira Abercromby, Ph.D. Associate Professor of Aerospace Engineering

COMMITTEE MEMBER: Kristina Jameson, Ph.D. Space Systems Loral

COMMITTEE MEMBER: Jordi Puig-Suari, Ph.D. Professor of Aerospace Engineering

COMMITTEE MEMBER: Kim Shollenberger, Ph.D. Professor of Mechanical Engineering 


\begin{abstract}
Thermal Modeling and Validation Testing of a Miniature Xenon Ion Thruster Samuel R. Parker
\end{abstract}

To support the research of miniature ion propulsion, Cal Poly's Miniature Xenon Ion (MiXI) thruster has been modeled and tested. Using ANSYS Fluent, a twodimensional model of the transient thermal environment of MiXI-CP-V3 was created. Validation testing was conducted in order to assess the accuracy of the thermal model. During testing, temperature was measured at eight locations across MiXI. From the test data it was determined that the thermal model did a poor job of predicting temperature due to incident radiation at the four locations nearest the keeper electrode. The four remaining locations were affected mostly by conduction and followed a trend that closely resembled the test data. The difference between the test data and the model's predicted temperature varied depending on time and location; the difference between the predicted data and the test data fell within $10{ }^{\circ} \mathrm{C}$, for most of the operation but reached $27.1^{\circ} \mathrm{C}$ at one location. The thermal model was used to assess ways to shunt heat transfer to the permanent magnets in order to postpone demagnetization, which occurs at $300^{\circ} \mathrm{C}$. Sheathing the entire keeper electrode with Macor was shown to reduce the temperature by as much as $13.3^{\circ} \mathrm{C}$ at certain times and locations. Due to its lower thermal conductivity, it was hypothesized that a thruster made of titanium would impede heat transfer to the magnets, however, the model showed an increase in temperature rise when properties of certain titanium alloys are applied. Applying a thermally insulative coatings to the anode was considered, but not modeled because the available coatings have a melting point below $350^{\circ} \mathrm{C}$. 


\section{ACKNOWLEDGMENTS}

I owe a huge debt of gratitude to my thesis committee, Dr. Abercromby, Dr. Shollenberger, Dr. Puig-Suari, and Dr. Jameson, for all of their help and advice throughout this project. I am sincerely grateful for all of their assistance, without which I could have never completed this project. I would also like to thank Dr. Goebel of JPL for answering my endless stream of questions and for always being so generous with his time. Dr. Goebel's knowledge on the subject of EP was invaluable to my efforts and I am extremely grateful for his help. I also owe David Knapp, Scott McGrail, and Max Glicklin a debt of gratitude for helping me troubleshoot MiXI and the vacuum chamber. Additionally, thank you to the Cal Poly Aerospace Engineering Department for providing me with such an excellent education. Finally, I would like to thank my father, Dave, and my mother Bridget for their love and support. 


\section{TABLE OF CONTENTS}

LIST OF TABLES X X

LIST OF FIGURES X xii

1 Introduction 1

1.1 Electric Propulsion Background . . . . . . . . . . . . . 2

1.2 Miniature Xenon Ion Thruster (MiXI) . . . . . . . . . . 5

1.2.1 MiXI-CP-V1 ................. 7

1.2.2 MiXI-CP-V2 . . . . . . . . . . . . . 8

1.2 .3 MiXI-CP-V3 .................. . . . 9

1.3 The Thermal Problem . . . . . . . . . . . . . . . . 10

1.4 Motivation .......................... 12

2 System Configuration $\quad 14$

2.1 The Hollow Cathode . . . . . . . . . . . . . . . . . . . 14

2.2 MiXI-CP-V3. . . . . . . . . . . . . . . . . . . 18

2.2.1 The Anode .................... 19

2.2.2 The Anode Pole Piece . . . . . . . . . . . . . 20

2.2.3 The Anode Cap Piece . . . . . . . . . . . . . 20

2.2.4 The Lower Magnet Mount \& Magnet Shield . . . . . . . . 21

2.2.5 The Grid Isolation Mount . . . . . . . . . . . . . . 22

2.2.6 The Cathode Isolation Sheath . . . . . . . . . . . . 23

2.2.7 The Plasma Confinement Magnets . . . . . . . . . . . . . 24

2.2.8 The Screen and Accelerator Grids . . . . . . . . . . . . . . 26

2.3 Final Assembly . . . . . . . . . . . . . . . . . . . . . 28

3 Testing $\quad 32$ 
3.1 Apparatus . . . . . . . . . . . . . . . . . . . 32

3.1 .1 Vacuum Chamber . . . . . . . . . . . . . . . . . 32

$3.1 .2 \quad$ Electrical System . . . . . . . . . . . . . . . . 34

3.1 .3 Propellant System . . . . . . . . . . . . . . . . 37

3.1.4 Data Acquisition . . . . . . . . . . . . . . 38

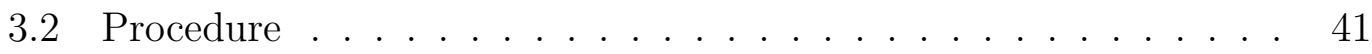

3.2 .1 MiXI Operation . . . . . . . . . . . . . . . 43

3.3 Data Reduction . . . . . . . . . . . . . . . . . . . . . 45

4 Thermal Model $\quad 51$

4.1 ANSYS Fluent . . . . . . . . . . . . . . . . . . 51

4.1 .1 Governing Equations . . . . . . . . . . . . . . . . 52

$4.1 .2 \quad$ P-1 Radiation . . . . . . . . . . . . . . . . . . 55

4.2 Geometry \& Mesh Generation . . . . . . . . . . . . . . 58

4.2 .1 Mesh Independence Study . . . . . . . . . . . . . . . 61

4.3 Thermal Model of MiXI-CP-V3 . . . . . . . . . . . . . . . . 62

4.3 .1 Assumptions . . . . . . . . . . . . . . . . . . 63

4.3.2 User Defined Functions . . . . . . . . . . . . . . . . . . 64

4.3.3 Thermal Contact Resistance . . . . . . . . . . . . . . 68

4.3.4 Material Properties . . . . . . . . . . . . . . . . . . 72

4.3.5 Solution Methods, Initialization, \& Calculation . . . . . 76

4.4 Results \& Validation . . . . . . . . . . . . . . . . . . . 78

5 Postponement of Magnet Overheating $\quad 84$

5.1 Model A: Isolate the Keeper Electrode . . . . . . . . . . . . . 84

5.1.1 Mesh Generation and Model Set Up . . . . . . . . . . . . 85

5.1 .2 Results . . . . . . . . . . . . . . . . . 86

5.2 Model B: Titanium Thruster . . . . . . . . . . . . . . . . . . . 89

5.2.1 Mesh Generation and Model Set Up . . . . . . . . . . . . 90

5.2 .2 Results . . . . . . . . . . . . . . . . . . . . . 90

5.3 Model C: Insulative Coatings _ . . . . . . . . . . . . . . 93

$\begin{array}{lll}6 & \text { Conclusion } & 95\end{array}$ 
6.1 Recommendations . . . . . . . . . . . . . . . . . . 96

6.2 Future Work . . . . . . . . . . . . . . . . . . . . . . . . 98

$\begin{array}{ll}\text { BIBLIOGRAPHY } & 101\end{array}$

$\begin{array}{ll}\text { APPENDICES } & 103\end{array}$

A User Defined Function 104

B Lessons Learned 108

B.1 Testing . . . . . . . . . . . . . . . . . . . . 108

C Plots of Test Data 112

$\begin{array}{ll}\text { D Procedures } & 119\end{array}$

D.1 High Vacuum Operation Procedure . . . . . . . . . . . . . . 119

D.1.1 Pre-Pumping Procedures and Safety Checks . . . . . . . 119

D.1.2 Cryopump Roughing Procedure . . . . . . . . . . . . 120

D.1.3 Martin-Victor Cycling Procedure . . . . . . . . . . . 120

D.1.4 Cryo Pump Compressor Procedure . . . . . . . . . . . 121

D.1.5 Experimental Loading Procedure . . . . . . . . . . . . 123

D.1.6 Chamber Roughing Procedure . . . . . . . . . . . . . . 124

D.1.7 Cryotorr Pumping Procedure . . . . . . . . . . . . . 124

D.1.8 Experiment Removal Procedure . . . . . . . . . . . 125

D.1.9 Shut Down Procedure . . . . . . . . . . . . . . . 125

D.1.10 Regeneration Procedure . . . . . . . . . . 126

D.1.11 Tips and Tricks . . . . . . . . . . . . . . . . . 127

D.2 Hollow Cathode Operating Procedures . . . . . . . . . . 128

D.2.1 Pre-Setup Procedure . . . . . . . . . . . . . . . . 130

D.2.2 Hollow Cathode Loading Procedure . . . . . . . . . . 130

D.2.3 Electrical Setup Procedure . . . . . . . . . . . . . . . 131

D.2.4 Propellant System Setup Procedure . . . . . . . . . . . 134

D.2.5 Hollow Cathode Operation Procedure . . . . . . . . . 136

D.2.6 Troubleshooting, Tips, and Tricks . . . . . . . . . . . 139

D.3 MiXI-CP-V3 Procedures . . . . . . . . . . . . . . . . . . 142

D.3.1 Pre-Setup Procedure . . . . . . . . . . . . . . . . . 143 
D.3.2 MiXI-CP-V3 Loading Procedure . . . . . . . . . . . . . . . 144

D.3.3 Electrical System Setup Procedure . . . . . . . . . . . . . 145

D.3.4 Propellant System Setup Procedure . . . . . . . . . . . . . 148

D.3.5 MiXI Operating Procedure . . . . . . . . . . . 148

D.3.6 Troubleshooting, Tips, and Tricks . . . . . . . . . . . . 151 


\section{LIST OF TABLES}

3.1 Locations of the thermocouples that were used to record temperature data. . . . . . . . . . . . . . . . . . . 44 41

3.2 Thruster operating conditions. . . . . . . . . . . . . . . . 44

4.1 Results of the mesh independence study. The minor difference between the results of the final mesh and the fine mesh proves grid independence of the final mesh. . . . . . . . . . . . . . . 62

4.2 Contact conductance between surfaces of MiXI parts. . . . . . . 71

4.3 Thermal Properties of Materials[26, 12, 8, 13]. . . . . . . . . 73

4.4 Thermal Properties of Part Interfaces. . . . . . . . . . . . . . 73

4.5 Cell Zone and Boundary Conditions. . . . . . . . . . . . . . . . . 74

4.6 Emissivities of Exposed Surfaces[20, 13, 12]. . . . . . . . . . . 75

4.7 Theoretical Temperatures vs Experimental Temperatures. Theoretical temperatures have an approximate error of $1.34 \%$. . . . . 82

5.1 Temperatures at thermocouple locations as predicted by the original theoretical model, and the model that featured the extended

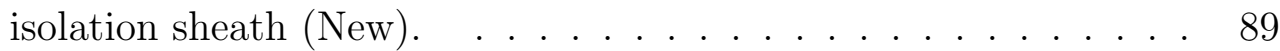

5.2 Thermal properties of titanium alloys[26, 25]. . . . . . . . . . . 90

5.3 Temperatures at thermocouple locations as predicted by the original theoretical model, and the model that featured ASTM Grade 9 titanium alloy $(\mathrm{New}) . \ldots . . \ldots 9 . \ldots 92$

5.4 Temperatures at thermocouple locations as predicted by the original theoretical model, and the model that featured IMI 685 titanium alloy. ....................... 93

D.1 Electrical Equipment Required for Hollow- Cathode . . . . . . . . 129

D.2 Hollow Cathode Propellant System . . . . . . . . . . . . . . . . . 129 
D.3 Electrical Equipment Required for Hollow- Cathode . . . . . . . . 142

D.4 Hollow Cathode Propellant System . . . . . . . . . . . . . . . . . 143 


\section{LIST OF FIGURES}

1.1 Illustration of a Hall effect thruster's operation . . . . . . . . . . . 3

1.2 Illustration of a Gridded Ion thruster's operation. . . . . . . . . . 5

1.3 An artists depiction of a 3U CubeSat that incorporates MiXI. . . 7

1.4 Cal Poly's first Miniature Xenon Ion thruster, MiXI-CP-V1. . . . 8

1.5 Cal Poly's second iteration of MiXI, MiXI-CP-V2. . . . . . . . . 9

1.6 MiXI-CP-V3 fully assembled and integrated with a $1 / 4$ " hollow cathode . . . . . . . . . . . . . . . 10

2.1 Two cutaway views of a hollow cathode. . . . . . . . . . . . 16

2.2 MiXI-CP-V3's 1/4" Hollow Cathode. . . . . . . . . . . . . . . . . 17

2.3 A cutaway view of the fully assembled MiXI-CP-V3 (screen and accel grids are not shown). . . . . . . . . . . . . . . 18

2.4 MiXI's Anode. . . . . . . . . . . . . . . . . . . . . . . 19

2.5 MiXI's anode pole piece. . . . . . . . . . . . . . . 20

2.6 MiXI's anode cap piece. . . . . . . . . . . . . . . . . . . 21

2.7 This image depicts 3D models of the lower magnet mount and the magnet shield. . . . . . . . . . . . . . . . . . . . . 22

2.8 MiXI's Grid Isolation Mount. . . . . . . . . . . . . . . . . . . 23

2.9 The cathode isolation sheath in its intended location at the end of the keeper electrode. . . . . . . . . . . . . . . . . . . . 24

2.10 A 2D cutaway view of MiXI reveals the location of the three rings of magnets (magnet size is not to scale). . . . . . . . . . 25

2.11 This image shows the location of the plasma confinement magnets within MiXI. . . . . . . . . . . . . . . 26

2.12 MiXI's Screen and Accelerator grids. . . . . . . . . . . . . 27

2.13 Placement of Screen and Accel Grids. . . . . . . . . . . . . . . . . 28 
2.14 Exploded view of MiXI. Not shown are the grids and fasteners. . .

2.15 This image shows the two MiXI-Hollow Cathode configurations that have been used for MiXI-CP-V3. . . . . . . . . . . . . 30

3.1 Minimum Atmospheric eXperimentation (MAX) Chamber in Cal Poly's Spacecraft Environments Laboratory. . . . . . . . . . . . .

3.2 A schematic of the MAX vacuum chamber apparatus in the Cal Poly Space Environments Laboratory. . . . . . . . . . . . . . . . . 34

3.3 Circuit diagram of MiXI's elelctrical system. . . . . . . . . . . . . 36

3.4 The portion of the propellant system that remains outside of the vacuum chamber. . . . . . . . . . . . . . . . 38

3.5 The NI Data Acquisition Chassis that was used for testing. . . . . 39

3.6 One of the two NI 9211 Thermocouple Input Module that was used for testing. . . . . . . . . . . . . . . . .

3.7 This figure shows the location of each thermocouple that was used to collect temperature data during MiXI's operations. . . . . . . .

3.8 All of the data that was collected during the 11 tests. The data from the first test is shifted to the right of all subsequent tests because it took several minutes longer for the keeper to strike. Therefore, data from this test was considered to be an out-lier and thrown out of final calculations. This data has been smoothed using MATLAB's built-in 'smooth' function. . . . . . . . . . . . .

3.9 Original data from tests $2-11$ before it had been smoothed. Notice the small spikes in thermocouples 5-8 between 16 and 23 minutes. These spikes are due to arcing throughout the chamber during operation of the thruster. . . . . . . . . . . . . . .

3.10 The data from tests 2-11 after being smoothed using MATLAB's 'smooth' function. . . . . . . . . . . . . . . . . . . . 48

3.11 The mean of the smoothed data from tests $2-11$. This is the data that will be used to validate MiXI's thermal model. . . . . . . . .

3.12 The mean of the smoothed data from tests 2-11 with error bars. The error bars indicate one standard deviation from the mean at that point in time. ................

4.1 This image shows the mesh that was created in ICEM and read into Fluent. MiXI can be seen in the bottom-center of the image, the orange area surrounding MiXI is the interior of the vacuum chamber. . . . . . . . . . . . . . . . 59 
4.2 A close-up view of the mesh area containing MiXI with each part labeled. . . . . . . . . . . . . . . . 60

4.3 Thermal conductivity of stainless steel 303 as a function of temperature......................... 67

4.4 Thermal conductivity of Macor as a function of temperature. . . . 68

4.5 A close-up view of two solid surfaces in contact. The arrows illustrate how heat is transfered between the surfaces. The gaps between the surfaces is called the void space. . . . . . . . . .

4.6 A close-up view of the mesh area containing MiXI with each part labeled. ................... . . . . 70

4.7 A cutaway view of a hollow cathode without the keeper electrode. 76

4.8 This image shows the temperature at each thermocouple location plotted against time for the heating phase of thruster operation. . $\quad 77$

4.9 A contour plot of MiXI's temperature profile after the heating phase, indicating what boundary conditions were changed at that time. . . . . . . . . . . . . . . . 78

4.10 Contour plot illustrating the temperature profile of MiXI after the heating phase of operations. . . . . . . . . . . . . . . 79

4.11 Contour plot illustrating the temperature profile of MiXI at time of cathode shutdown. . . . . . . . . . . . . . 80

4.12 This image shows the temperature at each thermocouple location plotted against time. . . . . . . . . . . . 81

4.13 This image shows the temperature at each thermocouple location plotted against time. . . . . . . . . . . . 82

5.1 The mesh of the expanded keeper isolator. . . . . . . . . . . . . 86

5.2 Contour plot showing the temperature profile of MiXI if the isolation sheath were extended. . . . . . . . . . . 87

5.3 Contour plot showing the temperature profile of MiXI if the isolation sheath were extended. . . . . . . . . . . . . 88

5.4 Contour plot showing the temperature profile of MiXI if it were constructed of IMI 685 titanium alloy. . . . . . . . . . . . . . . . 91

5.5 Contour plot showing the temperature profile of MiXI if it were constructed of IMI 685 titanium alloy. . . . . . . . . . . . . . 92

C.1 Test 1. . . . . . . . . . . . . . . . . . . . 112 


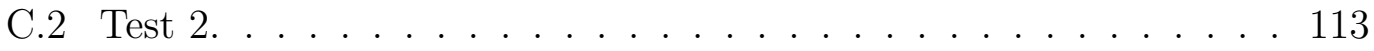

C.3 Test 3. . . . . . . . . . . . . . . . . . . . . . . . . 113

C.4 Test 4. . . . . . . . . . . . . . . . . . . . . . 114

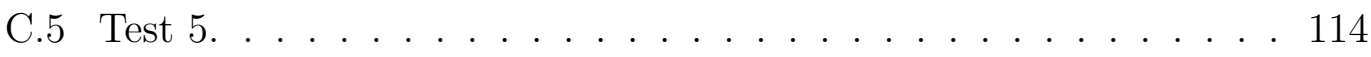

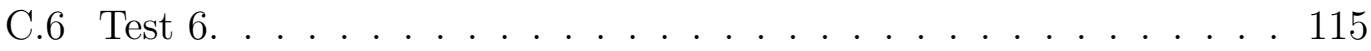

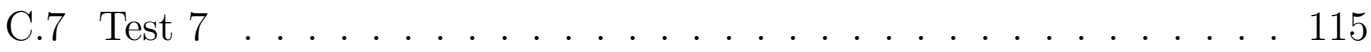

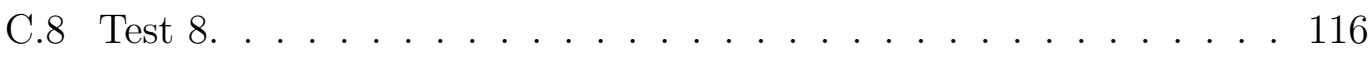

C.9 All of the data that was collected during the 11 tests. The data from the first test is shifted to the right of all subsequent tests because it took several minutes longer for the keeper to strike. Therefore, data from this test was considered to be an out-lier and thrown out of final calculations. . . . . . . . . . . . . . . 116

C.10 All test data, excluding outliers. . . . . . . . . . . . . . . 117

C.11 The average of the 10 tests that were considered. . . . . . . 117

C.12 The average of the 10 tests that were considered, with error bars to indicate one standard deviation from the mean at a point in time.118

C.13 One standard deviation from the mean vs time. . . . . . . . . 118

D.1 Hollow Cathode Circuit Diagram. . . . . . . . . . . . . . . 131

D.2 Heater and Keeper attachment points. . . . . . . . . . . 132

D.3 Hollow Cathode Propellant System Layout . . . . . . . . . . 135

D.4 MiXI/Hollow Cathode Propellant System. Notice that there is no diaphragm valve between the flow meter and the propellant feedthrough into the chamber. . . . . . . . . . . . . 136

D.5 Hollow cathode during nominal operation. Notice the distinct cone shape of the plasma. . . . . . . . . . . . . . . . 140

D.6 MiXI test setup. . . . . . . . . . . . . . . . . . . . 145

D.7 MiXI Circuit Diagram. . . . . . . . . . . . . . 146 


\section{Introduction}

Since the beginning of the space race, the development of highly efficient propulsion systems for spacecraft has been a top priority. The thrust required to maneuver a spacecraft has traditionally been supplied by chemical propulsion systems; however, electric propulsion (EP) systems have become increasingly popular in the past decade. In contrast to chemical propulsion, EP systems produce a relatively small amount of thrust (0.01-700 mili-Newtons) but have a very high specific impulse $(>1000 \mathrm{sec})[10]$. The higher specific impulse means that EP systems require less payload mass be dedicated to propellant, resulting in a significant cost savings[24].

The first regular use of spacecraft electric propulsion began in 1971 with the launch of the Meteor satellite by the Soviet Union [10]. This spacecraft was the first to utilize the Soviet-developed Hall thruster, which was used for station keeping on communication satellites. Since then, hundreds of Hall thrusters have been launched aboard dozens of Soviet and Russian spacecraft. The use of ion propulsion (a subset of electric propulsion) by the United States began in 1997 with the launch of the Hughes PAS-5 satellite, which utilized the Hughes-

developed Xenon Ion Propulsion System (XIPS) for station keeping maneuvers [10]. Since then the use of electric propulsion on spacecraft, specifically gridded ion thrusters, has steadily increased.

The recent successes of gridded ion and Hall thrusters has lead to increased 
interest in micro ion propulsion. The goal of micro ion propulsion is to take larger ion thrusters and scale them down. Research laboratories and universities have had success in recent years developing miniature ion and Hall thrusters that can produce $0.01 \mathrm{mN}-10 \mathrm{mN}$ of thrust while still maintaining high specific impulse [27]. These thrusters would be ideal for missions that require highly precise attitude control. Additionally, the small physical size of the thrusters $(3 \mathrm{~cm})$ also makes them very attractive for large (3U) CubeSat missions. While there are several possible applications for micro ion propulsion, thrusters of this size have proven to be difficult to develop. To date, no micro ion thrusters have ever been flown.

\subsection{Electric Propulsion Background}

Spacecraft electric propulsion is an umbrella term that includes three distinct concepts: electrothermal propulsion (arcjet, resistojet), electrostatic propulsion (Hall thruster, gridded ion thruster), and electromagnetic propulsion (pulsed plasma thruster, magnetohydrodynamic thrusters) [10, 14]. Of these concepts, electrostatic propulsion has become the most common. Electrostatic propulsion generates thrust by ionizing an inert gas then accelerating the ions via an electric field[10].

Like most electrostatic propulsion systems, modern Hall thrusters typically use xenon gas as propellant[10, 24, 11]. As fig. 1.1 illustrates, Hall thrusters inject this propellant into a cylindrical channel. The neutral xenon atoms are ionized by electron bombardment as they enter the channel. Electrons are provided by a hollow cathode that is positioned just outside the exit of the thruster. The electrons produced by the cathode are drawn into the channel by a positively 
charged anode that is positioned at the channel's inlet, where the propellant is injected. As the electrons flow from the cathode to the anode, they encounter a magnetic field perpendicular to the direction of flow. What happens next can be described by the Hall effect, which states that a charged particle moving in a vacuum is deflected sideways by a magnetic field[16]. The magnetic field causes the electrons to form an electric field that electrostatically accelerates the positively charged ion particles. Thrust is the result of the ions being accelerated to very high exhaust velocities by the electric field[10].

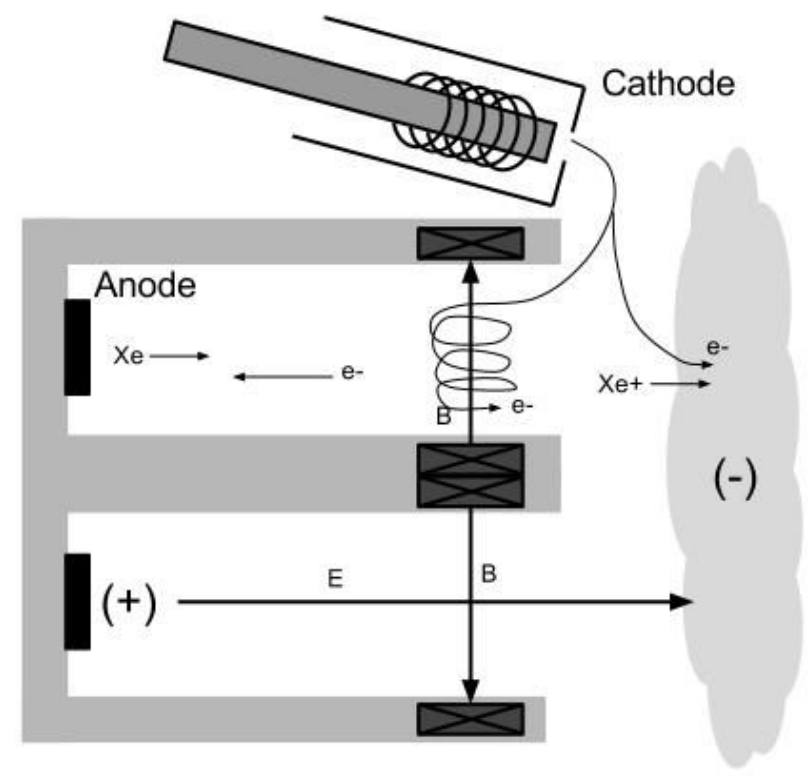

Figure 1.1: Illustration of a Hall effect thruster's operation.[19]

Ion thrusters follow the same basic concept of producing thrust by electrostatically accelerating ionized gas. The physics behind ion thrusters is simpler than that of Hall thrusters, but ion thrusters are mechanically more complex. The operation of ion thrusters is illustrated by fig. 1.2. Ion thrusters inject propellant into a discharge chamber where it can be ionized by electrons[10]. In modern thrusters, these electrons are introduced into the discharge cham- 
ber via a hollow cathode that is positioned at the chamber's inlet. Due to its relatively large atomic mass and low ionization energy, xenon gas is typically used as propellant[15]. As it exits the cathode, is then ionized by electron bombardment $[10,15]$. The electrons introduced by the cathode, as well as the secondary electrons that are released by xenon atoms during the ionization process, are attracted to the walls of the discharge chamber. The wall of the discharge chamber is positively biased and acts as an anode for the hollow cathode. In order to keep most of the electrons in the the chamber, magnets are placed on the external side of the discharge chamber walls. The resulting magnetic field redirects the electrons back into the discharge chamber where they can continue bombarding xenon atoms[10]. The more time an electron spends in the chamber, the more time it has to ionize a xenon atom, and the more efficient the ionization process becomes[9]. The positively charged xenon ions are then accelerated by a series of electrically biased grids at the exit of the thruster. These grids contain numerous coaxial apertures, through which the xenon ions flow[10]. The upstream grid is called the screen grid and has a highly positive voltage bias. The downstream grid is called the accelerator (accel) grid and carries a negative bias. The positive xenon ions in the ionization chamber are extracted by the grids and accelerated to high velocities through the apertures[10]. Outside the exit of the thruster, another hollow cathode provides electrons to the thruster's ion beam. These electrons neutralize the positively charged particles in the beam[10, 15]. 


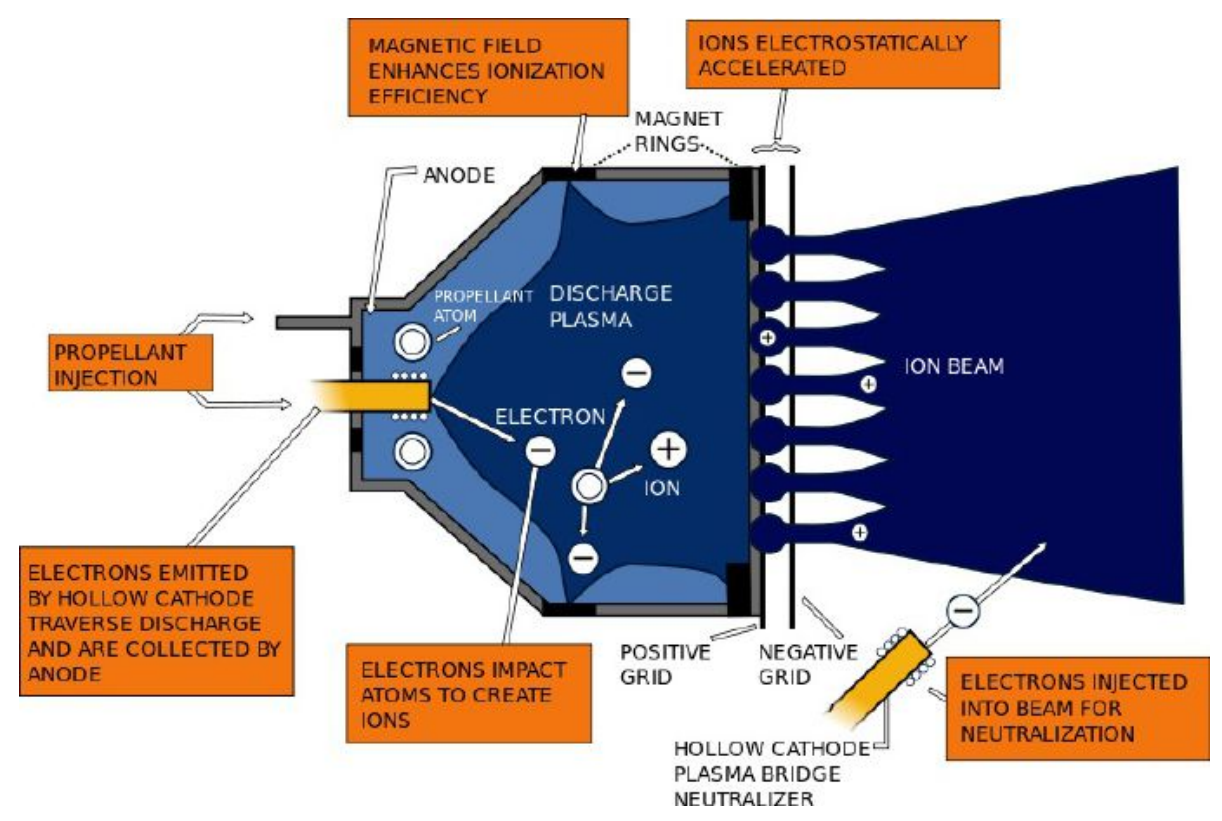

Figure 1.2: Illustration of a Gridded Ion thruster's operation.[9]

\subsection{Miniature Xenon Ion Thruster (MiXI)}

The Miniature Xenon Ion Thruster (MiXI) is a micro ion propulsion thruster that is $3-\mathrm{cm}$ in diameter, produces a thrust on the order of $0.5-1.5 \mathrm{mN}$, and can achieve a specific impulse of over 3000s[15]. The first MiXI thruster was developed in the early 2000's at NASA's Jet Propulsion Laboratory[27]. MiXI was designed to achieve an efficiency of $>50 \%$ while requiring a relatively low amount of power $(<50 \mathrm{~W})$. Full sized ion thrusters typically require thousands of Watts of power to operate, which limits their use to large satellites capable of meeting the power requirements of the truster. The low power requirements of MiXI make it an ideal candidate for precision attitude control of small to medium sized satellites. The power requirements and physical size of MiXI make it a potential primary propulsion system for picosatellites (also known as CubeSats)[15].

Currently, there are several mission concepts that propose using Miniature 
Xenon Ion thrusters. One of these concepts is the Terrestrial Planet Finder (TPF)-Emma mission. TPF-Emma is currently a JPL mission candidate that would find and study planets outside the solar system[23]. The mission concept consists of five spacecraft flying in formation; to meet the performance requirements, the spacecraft formation will need to maintain one-centimeter accuracy. In order to accomplish this, the mission designers have proposed placing several thruster pods, each containing four MiXI thrusters, on each vehicle[23, 15, 27].

In addition to the TPF-Emma mission, there has recently been some interest in integrating MiXI into a CubeSat. CubeSats are simple, miniaturized spacecraft buses that offer a low cost option for space research. A typical, $1 \mathrm{U}$ CubeSat is a $10 \mathrm{~cm} \times 10 \mathrm{~cm} \times 10 \mathrm{~cm}$ cube that has a mass of $1 \mathrm{~kg}$; they are launched as a secondary payload and are deployed (up to three at a time) from the launch vehicle via the Poly Picosatellite Orbital Deployer (P-Pod). They typically do not have a propulsion system on board and are stuck in the orbit they deploy into. MiXI has been proposed as a primary propulsion system for CubeSats however, a $1 \mathrm{U}$ CubeSat does not have the volume or power required to operate a MiXI thruster. A 3U configuration (essentially three 1U CubeSats stacked on top of each other) with deployable solar panels would produce enough power to operate MiXI. A 3U system would also have enough volume to contain MiXI and its support equipment. Figure 1.3 shows an illustration of a $3 \mathrm{U}$ CubeSat with deployable solar panels $[7,15,27]$. 


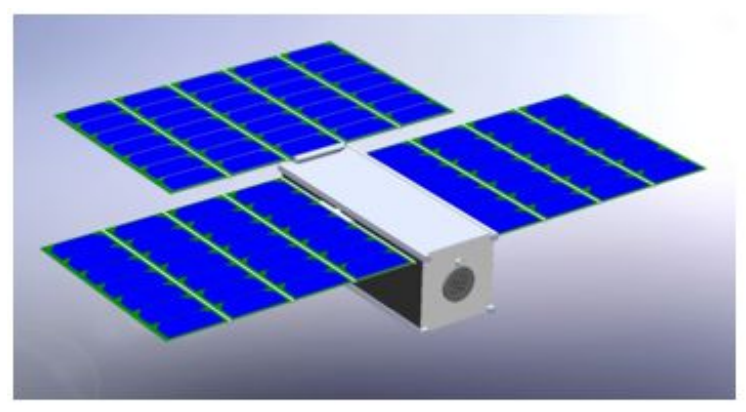

Figure 1.3: An artists depiction of a $3 \mathrm{U}$ CubeSat that incorporates MiXI.[7]

While there are many potential applications of micro ion propulsion, scaling down ion thrusters has proved to be quite challenging. Because of its small size and low power requirements, MiXI needs to see an improvement in heat management before it can become a viable flight thruster.

\subsubsection{MiXI-CP-V1}

The history of MiXI at Cal Poly began in the late 2000's with the development of MiXI-CP-V1[27]. MiXI-CP-V1 was developed by graduate student Coleman Younger with the assistance of Dr. Richard Wirz of UCLA, Dr. Dan Goebel of JPL, and Dr. Kristina Jameson of Cal Poly. This iteration of MiXI was based on the design for Dr. Wirz's micro ion thruster(the prototype for MiXI) and utilized a coiled tungsten filament cathode (CTFC) for electron bombardment. Mr. Younger tested the thruster in order to validate a thermal model he had developed, he then used the model to assess the feasibility of various heat management techniques. This thermal model was created using the SolidWorks software package and was validated using temperature data that was collected during testing[27]. MiXI-CP-V1 is shown in fig 1.4. 


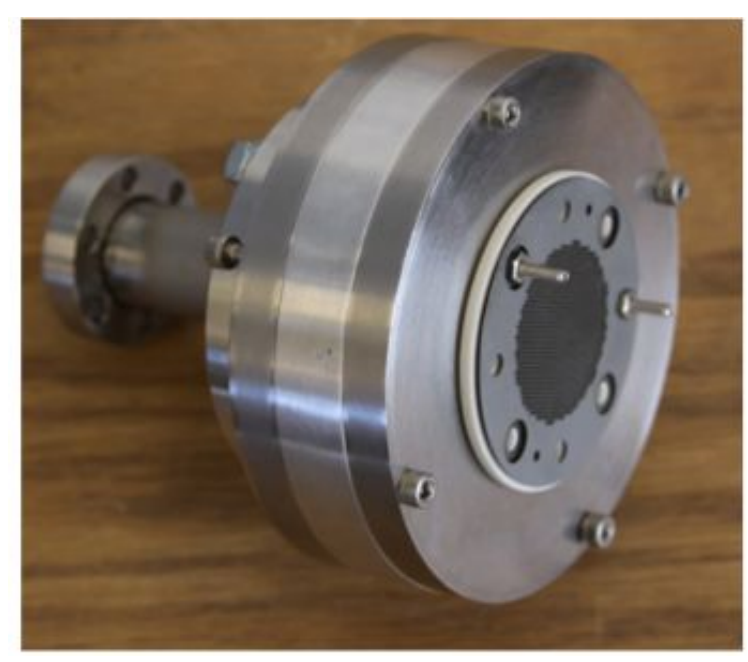

Figure 1.4: Cal Poly's first Miniature Xenon Ion thruster, MiXI-CPV1[27].

\subsubsection{MiXI-CP-V2}

Cal Poly's MiXI program continued in 2011 with the development of MiXI-CPV2[15]. This iteration of MiXI was created by David Knapp with the assistance of Dr. Jameson, Dr. Goebel, and Dr. Wirz. MiXI-CP-V2 was based on the original design of MiXI and utilized a CTFT for plasma generation. Argon was used as propellant rather than Xenon due to it's low cost, however, the lower mass and higher ionization energy of Argon means it is less efficient than Xenon[15].

This version of MiXI was successfully tested in Cal Poly's vacuum chamber facilities and was capable of acheiving $0.3-0.7 \mathrm{mN}$ of thrust, $1800-3500$ s of specific impulse, and a 15-40\% mass utilization efficiency[15]. The thruster's relatively low efficiency, thrust, and Isp can be attributed to the use of Argon as propellant, and a tungsten filament cathode rather than a hollow cathode. Additionally, Mr. Knapp concluded that more in-depth design and analysis of the magnetic field could have improved plasma confinement as well as overall performance[15]. 


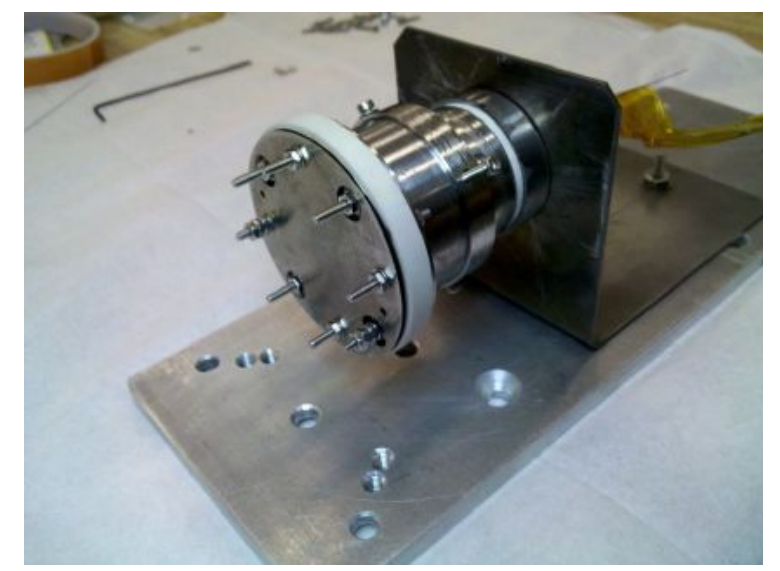

Figure 1.5: Cal Poly's second iteration of MiXI, MiXI-CP-V2[15].

\subsubsection{MiXI-CP-V3}

Cal Poly MiXI-CP-V3 is the latest iteration of MiXI. Developed by David Knapp in 2012, MiXI-CP-V3 is the first MiXI thrusters to incorporate a hollow cathode for ion bombardment rather than a CTFC[15]. This version of MiXI was built to help identify and investigate more of the issues that arise when integrating a hollow cathode with a miniature ion thruster[15].

MiXI-CP-V3 was designed around a small, 1/4" cathode and built upon the lessons learned from its immediate predecessor, MiXI-CP-V2. In depth analysis of the required magnetic fields supported the decisions regarding magnet selection and placement. Additionally, Xenon propellant was used instead of Argon. These factors, combined with the use of a hollow cathode rather than a CTFC, improved most of MiXI's performance parameters over its predecessor. MiXICP-V3 produced a thrust of 1.2-2.0 $\mathrm{mN}$, a specific impulse of $1500-3300 \mathrm{~s}$, and a mass utilization efficiency of $30-77 \%$ (the range of these parameters is due to the variation in mass flow rate and discharge current). While the specific impulse isn't as high as MiXI-CP-V2, CP-V3 was a success and is continuing to serve 
its intended purpose: acquiring insight into the challenges of miniature electric propulsion and "advancing the MiXI design" [15].

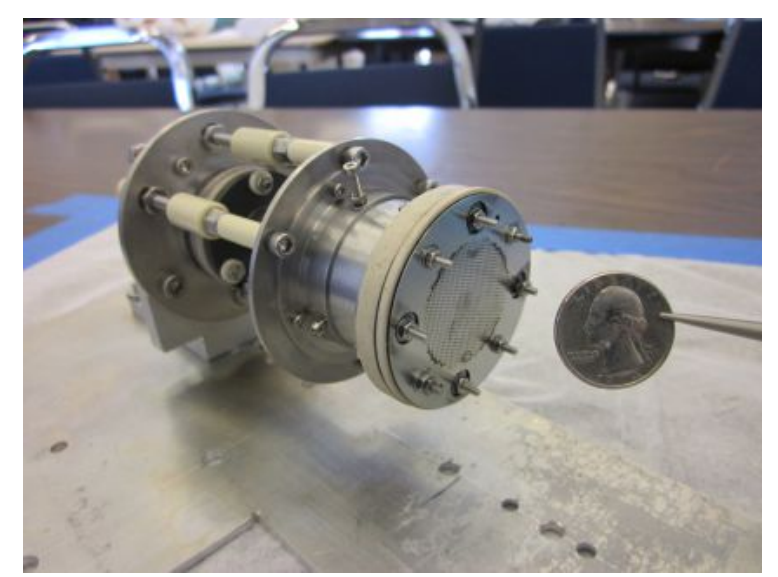

Figure 1.6: MiXI-CP-V3 fully assembled and integrated with a 1/4" hollow cathode[15].

\subsection{The Thermal Problem}

The process of plasma generation introduces a large amount of heat into ion thrusters. Excessive heat can cause several problems for the thruster, such as accelerated degradation of the grids, out-gassing of the electro-isolators, and demagnetization of the permanent magnets[27]. The maximum service temperature of MiXI's Samarium-Cobalt the permanent magnets is $300^{\circ} \mathrm{C}$, the lowest maximum temperature of any component of MiXI. At $300^{\circ} \mathrm{C}$, the ability of the magnets to produce a strong magnetic field is compromised and the efficiency of the thruster drops drastically due to the lack of electron confinement. The magnetic field strength can be restored by allowing the magnets to cool to temperatures below their maximum working temperature; however, if the temperature of the magnets becomes too great the physical properties of the magnets will be forever changed and they will no longer produce a magnetic field[16, 15]. The tempera- 
ture at which this occurs is known as the Curie temperature[17]. Other magnetic materials such as Aluminum-Nickel-Cobalt have much higher maximum working temperatures, but they're not used on EP thrusters because the strength of their magnetic field is too low.

Full sized flight thrusters are not as sensitive to high temperatures as miniature ion thrusters. First of all, full sized ion and Hall thrusters (such as the XIPS and NEXT) utilize electromagnets for electron confinement[10]. The magnetic field produced by electromagnets does not lose strength as their temperature increases. Using electromagnets on MiXI would increase the thruster's power requirements, thereby failing to meet the goal of a small, low power ion thruster[27, 15].

Additionally, larger $(15-30 \mathrm{~cm})$ thrusters have more material for heat to propagate through and a larger surface area for the heat to radiate from. As a result, these thrusters can be operated for longer periods of time because it takes much longer for heat to reach temperature-sensitive components and the thruster can radiate more of this heat out into space. MiXI, on the other hand, is inherently less capable of handling the heat input due to its small physical size. The low thrust that ion thrusters produce $(1-250 \mathrm{mN})$ means that they must be operated continuously for an extended period of time to impart the required delta- $\mathrm{V}$ onto a spacecraft. Current flight-ready thrusters are capable of operating for days, weeks, or even years without being cycled or shut down. In a laboratory setting, MiXI is usually operated for a period of 5-10 minutes before the maximum temperature of the magnets is reached and the thruster must be shut down.

The ultimate goal of MiXI thermal analysis is to develop a way for MiXI to operate indefinitely without the magnets becoming overheated. This thesis hopes to contribute to these efforts by test firing MiXI and collecting temperature data 
which will be used to create and validate a thermal model of MiXI. If this model is accurate enough, it will then be used to determine what can be done to postpone the onset of magnet overheating. Postponement of magnet overheating is a crucial step towards extending the burn time of MiXI.

\subsection{Motivation}

Before MiXI can be operated on board a spacecraft, it must demonstrate the ability to manage heat in a laboratory setting. The motivation of this thesis is to develop a better understanding of MiXI's thermal operating conditions and explore ways to mitigate the adverse effects of overheating. This will be accomplished by developing a 2-dimensional thermal model of MiXI-CP-V3 and using it to assess several concepts of thermal management. The model will help determine what modifications to MiXI-CP-V3 will best mitigate the overheating problem. In order to verify that the thermal model is an accurate representation of reality, the thermal model will be compared to temperature data collected from MiXI-CP-V3.

There are many possibilities for mitigating the effects of overheating and thereby increasing MiXI's operating time, but building the hardware and testing these systems can be expensive. Generating computer models allows us to compare the potential of these systems and determine the best way to move forward, saving time and money in long run.

This thesis hopes to further advance the design of MiXI by evaluating the potential of various methods of postponing the onset of magnet degaussing. The thermal models developed for this thesis were developed for one unique version of MiXI however, the results and recommendations should be applicable to many 
other ion thrusters. The knowledge provided by this evaluation will hopefully help others continue the development of a thermal management system for MiXI. 


\section{System Configuration}

Since its inception in 2001, MiXI has been a work in progress; its design has progressed iteratively and no two thrusters are quite the same. Cal Poly is currently working with its third iteration of MiXI. MiXI-CP-V3 is based on JPL's original design and was developed and built by David Knapp in 2011[15]. This thruster has the same core elements of all MiXI thrusters: an anode, permanent magnets, screen and accel grids, and ceramic isolators. The things that make this thruster unique are the size, quantity, and placement of the permanent magnets, as well as the physical shape of the thruster. It is also the first version of MiXI at Cal Poly to utilize a hollow cathode for electron bombardment.

\subsection{The Hollow Cathode}

Ion thrusters generate plasma by ionizing a neutral gas propellant via electron bombardment[10]. In order to facilitate electron bombardment, ion thrusters utilize a cathode to emit electrons. Early ion thrusters and some modern laboratory thrusters use a Coiled Tungsten Filament Cathode(CTFC) as the electron emitter. A CTFC operates by passing a current through a coiled tungsten wire; when the tungsten wire becomes hot enough $(>2600 \mathrm{~K})$ it will emit the electrons required for ion bombardment[10]. Due to its simplicity, CTFC's are still popular in laboratory settings, however the heater power required to achieve adequate 
current densities renders this cathode relatively inefficient and impractical for flight thrusters[10].

Developed in the 1960's, hollow cathodes have largely replaced CTFC's for electron emission on ion and Hall thrusters[10]. As shown in fig. 2.1, a basic hollow cathode consists of a cathode tube, a cylindrical insert, an orifice plate, a heater, and a keeper electrode. Xenon propellant flows through the tube and is bombarded with electrons that are emitted from the insert. The cathode insert is the electron emitter and is usually made from tungsten impregnated barium oxide $(\mathrm{BaO}-\mathrm{W})$ or lanthanum hexaboride $\left(\mathrm{LaB}_{6}\right)[10]$. The insert is located at the end of the tube adjacent to the orifice plate. In order to increase the temperature of the insert to the temperatures required for electron emission, the tube is surrounded by a heater. The heater is usually a coaxial sheathed wire that is wrapped around the outside of the tube[10].

The heater, orifice plate, insert, and tube are all enclosed within a keeper electrode. This keeper electrode carries a positive bias during cathode operations to help draw electrons out of the cathode while deflecting ions near its exterior. The keeper serves three main purposes: initiating plasma discharge during startup, protecting the heater and orifice plate from ion bombardment, and to provide a thermal insulator for maintaining temperature within the cathode[10]. 


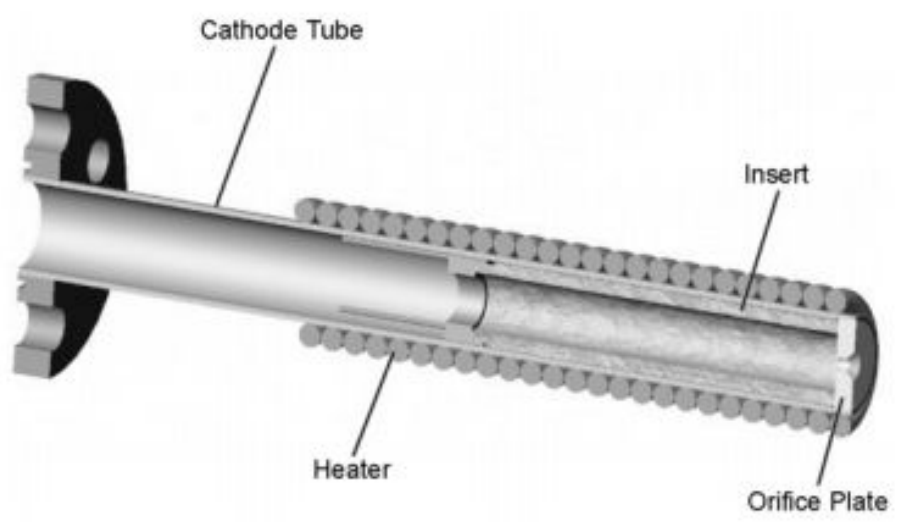

(a) A cutaway view of a hollow cathode without the keeper electrode[10].

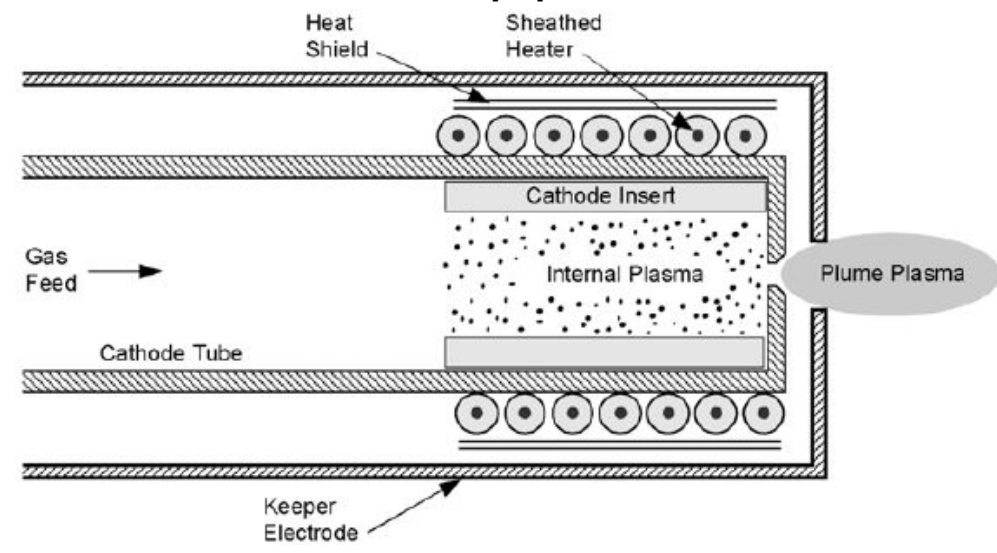

(b) A cutaway view of a hollow cathode with the keeper electrode[10].

Figure 2.1: Two cutaway views of a hollow cathode.

Hollow cathodes come in many shapes and sizes however, miniaturized hollow cathodes do not yet exist. Conventional hollow cathodes usually encounter instabilities at flow rates $\leq 1$ Standard Cubic Centimeter per Minute (SCCM) and since MiXI is designed to operate between 0.1 and 0.7 SCCM, this presents a problem[15]. Because CTFC's can operate at lower flow rates and are simpler and less expensive than hollow cathodes, previous versions of MiXI were forced to rely on CTFC's for electron bombardment. Research and development of miniaturized replacements for CTFC's is currently being conducted by the Wirz Research 
Group at UCLA[15]. However, a miniature hollow cathode optimized for MiXI operations has yet to be developed[10][15].

In 2012, David Knapp developed MiXI-CP-V3, the first attempt to incorporate a hollow cathode with MiXI[15]. MiXI-CP-V3 utilized a small, $6.25 \mathrm{~mm}$ (1/4 inch) diameter hollow cathode, similar to the cathodes used by the NSTAR thruster. This cathode, donated by JPL, is shown in fig. 2.2. Mr. Knapp tested this cathode in Cal Poly's Space Environments Laboratory and showed that it was capable at operating at flow rates as low as 0.5 SCCM. The use of this cathode on MiXI doubled the thruster's mass utilization efficiency and decreased its discharge loss, at the cost of increasing its power requirements and thrust beyond the original design parameters[15].

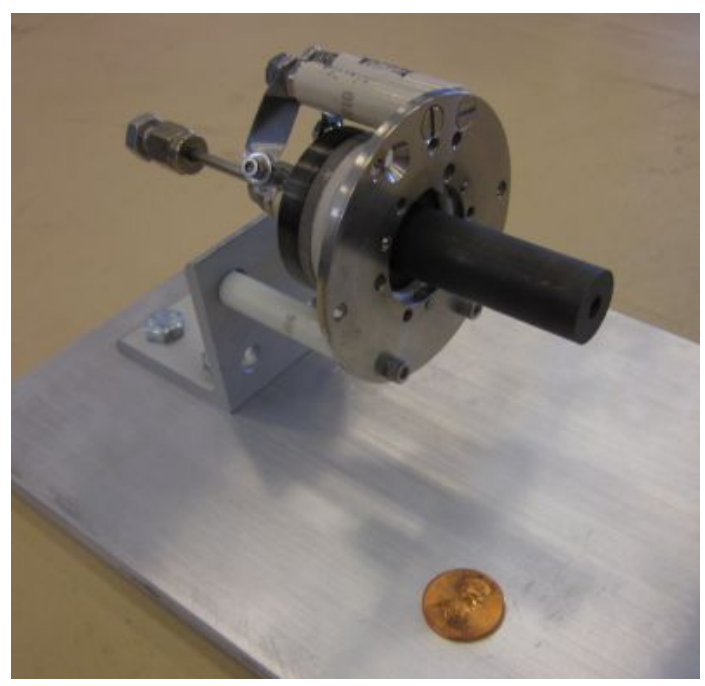

Figure 2.2: MiXI-CP-V3's 1/4" Hollow Cathode[15].)

MiXI-CP-V3's hollow cathode contains a tungsten impregnated barium oxide $(\mathrm{BaO}-\mathrm{W})$ insert[15]. Barium oxide inserts have a lower work function compared to lanthanum hexaboride $\left(\mathrm{LaB}_{6}\right)$ inserts, meaning that they require less heat $\left(1100{ }^{\circ} \mathrm{C}\right)$ to achieve the same electron emission as $\mathrm{LaB}_{6}$ inserts $(1300-$ 
$\left.1500^{\circ} \mathrm{C}\right)[10]$. However, the $\mathrm{BaO}-\mathrm{W}$ insert is much more susceptible to poisoning and requires high purity research grade (99.999\% pure) xenon or argon propellant. The cathode tube is wrapped in a coaxial insulated heater wire, and encased in a graphite electrode. The graphite keeper electrode is 0.7 inches in outer diameter and two inches long. It is mounted to a steel chassis that is 2.5 inches in diameter and 1 inch thick.[15]

\section{$2.2 \quad \mathrm{MiXI}-\mathrm{CP}-\mathrm{V} 3$}

As it's currently the only version of MiXI to utilize a hollow cathode, MiXI-CPV3 has a unique layout and design. The thruster consists of several individual parts, each of which will be discussed in this section.
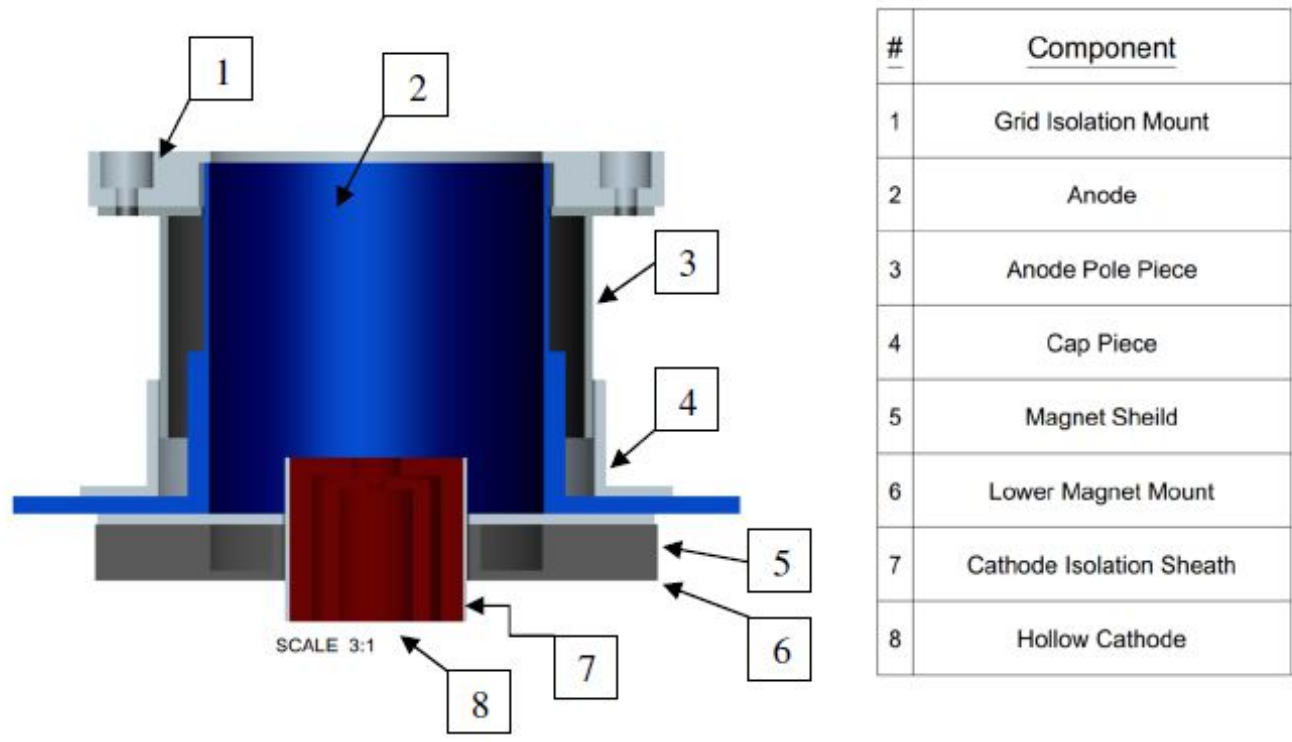

Figure 2.3: A cutaway view of the fully assembled MiXI-CP-V3 (screen and accel grids are not shown)[15]. 


\subsubsection{The Anode}

Made of 303 stainless steel, the anode has a $3 \mathrm{~cm}$ inner diameter and is $3 \mathrm{~cm}$ in length. The anode acts as the thruster's discharge chamber; a positive voltage bias applied to the anode walls helps to draw the electrons out of the cathode. Permanent magnets are placed around the exterior of the anode to deflect electrons away from the anode and back into the discharge chamber. Rather than having a uniform wall thickness, the walls of the anode follow a stepped configuration. The anode wall is only $0.5 \mathrm{~mm}$ thick at the magnet cusp location, but it increases to $1.5 \mathrm{~mm}$ once past the magnets. This design was chosen to increase the volume for thermal conduction, thereby postpone some of the negative effects of overheating.[15]

At one end of the anode there is a flange that serves as an attachment point for most of the other thruster components. This flange is $66 \mathrm{~mm}$ in diameter and 2mm thick. Figure 2.4 shows a solid model of the anode.[15]

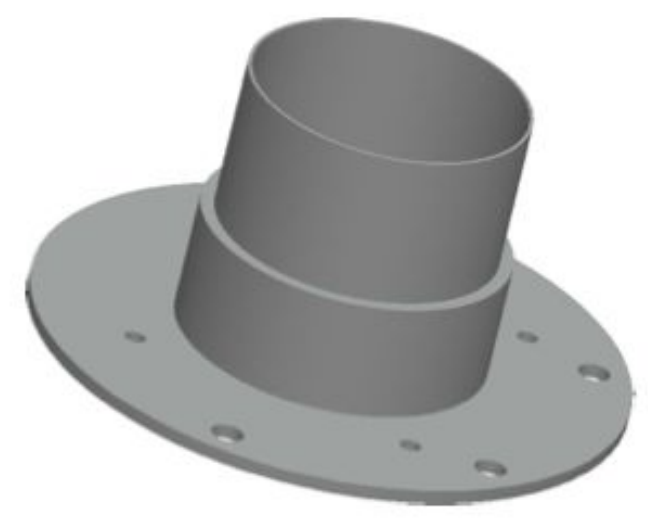

Figure 2.4: MiXI's Anode[15]. 


\subsubsection{The Anode Pole Piece}

The anode pole piece is a cylinder that provides an enclosure for the plasma confinement magnets. The pole piece is $21 \mathrm{~mm}$ long, $1 \mathrm{~mm}$ thick and is made from ferrous steel (state exact grade). The ferrous steel allows the magnets to attach to the pole piece without the use of additional mounting hardware. The inner diameter of the pole piece is designed to allow a stack of three $1 \mathrm{mmx} 3 \mathrm{~mm}$ disk magnets to fit between the pole piece and the anode. At one end of the pole piece, there is a $1 \mathrm{~mm}$ thick, $50 \mathrm{~mm}$ diameter flange. This flange is on toward the exit of the thruster, the side opposite to the anode's flange and provides an attachment point for the grid isolation piece. A solid model of the pole piece is shown in fig. 2.5.[15]

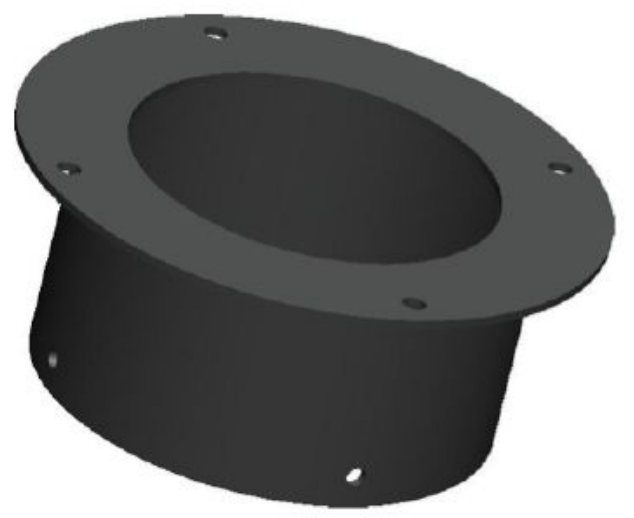

Figure 2.5: MiXI's anode pole piece[15].

\subsubsection{The Anode Cap Piece}

The anode cap piece is simply a bracket to attach the anode pole piece to the anode's flange. Shown in fig. 2.6, the cap piece is made of 303 stainless steel and is $10.525 \mathrm{~mm}$ in length. It's flange has an outer diameter of $53.45 \mathrm{~mm}$ while the 
cylindrical section has an outer diameter of $41.45 \mathrm{~mm}$ and an inner diameter of $39.45 \mathrm{~mm} .[15]$

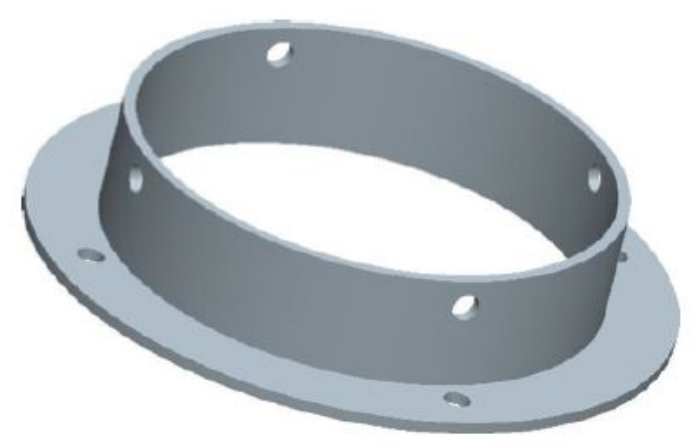

Figure 2.6: MiXI's anode cap piece[15].

\subsubsection{The Lower Magnet Mount \& Magnet Shield}

Like the anode pole piece, the lower magnet mount is designed to house some of the plasma confinement magnets. The lower magnet mount contains an additional ring of magnets at the rear of the thruster, near the hollow cathode. The magnet mount is made of ferrous steel which, like the pole piece, attracts the magnets and holds them in place without any extra mounting hardware. The magnet mount is a disk with a $50.26 \mathrm{~mm}$ outer diameter and a $16.26 \mathrm{~mm}$ hole bored into the center. The maximum thickness of this part is $5 \mathrm{~mm}$, but there is a $30 \mathrm{~mm}$ diameter countersink that reduces the thickness to $0.985 \mathrm{~mm}$ near the center. This countersink provides a $4.115 \mathrm{~mm}$-thick cavity that houses the lower ring of plasma confinement magnets.[15]

The magnet shield is a simple, $1 \mathrm{~mm}$ thick disk that's sandwiched between the lower magnet mount and the anode flange. It has same outer and inner diameter as the magnet mount but it's made with 303 stainless steel and therefore, 
it does not attract the confinement magnets. As its name suggests, the magnet shield exists to protect the magnets from the plasma within the discharge chamber. Sandwiched in the cavity between the shield and the magnet mount is a ring consisting of 20 stacks of SmCo magnets. Each stack contains four $1 \mathrm{mmx} 3 \mathrm{~mm}$ magnets and the stacks are form a ring around the center of the magnet mount.[15]

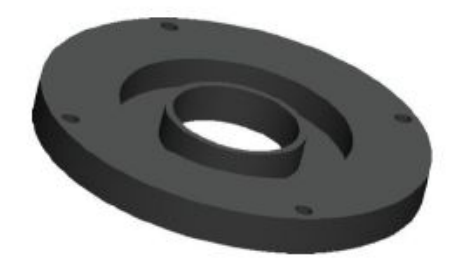

(a) Lower Magnet Mount[15].

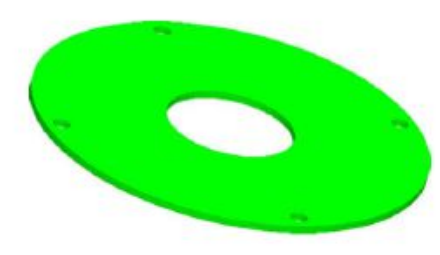

(b) Magnet Shield[15].

Figure 2.7: This image depicts 3D models of the lower magnet mount and the magnet shield.

\subsubsection{The Grid Isolation Mount}

The grid isolation mount is what separates the screen and accel grids from the rest of the thruster. The isolation mount is attached to the flange of the anode pole piece via four screws. The screws are guided through countersunk holes; the countersink prevents the heads of the screws from coming into contact with the grids. An additional four countersunk holes provide attachment points for the grids. In order to ensure electrical isolation the grid isolation mount is made of Macor, a machinable glass ceramic.[15] 


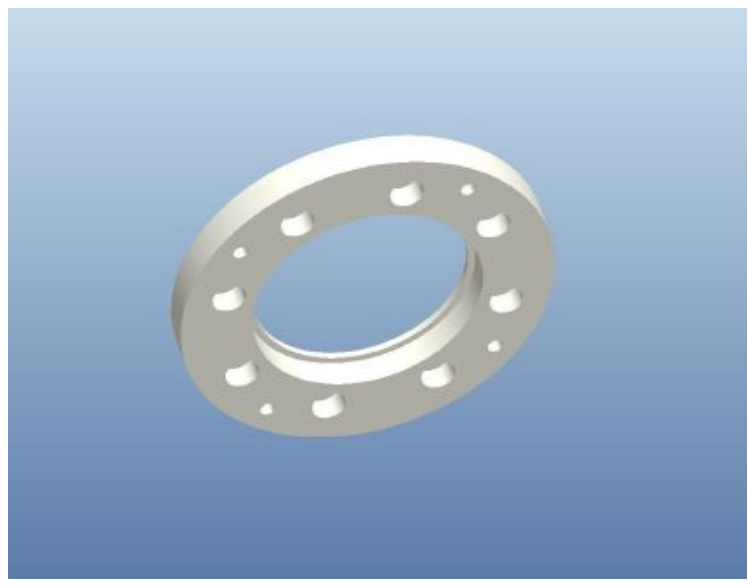

Figure 2.8: MiXI's Grid Isolation Mount.[15]

\subsubsection{The Cathode Isolation Sheath}

The cathode isolation sheath is a thin-walled ring that provides electrical insulation between the hollow cathode's keeper electrode and the rest of the thruster. The isolator has a length of $10 \mathrm{~mm}$, an outer diameter of $18 \mathrm{~mm}$, and a thickness of $1 \mathrm{~mm}$. Like the grid isolation mount, the cathode sheath is constructed of Macor. Figure 2.9 depicts the isolation sheath resting on the hollow cathode. Unlike the rest of the components of MiXI, the cathode isolation sheath is not fixed to any other parts and will sometimes slide out of position.[15] 


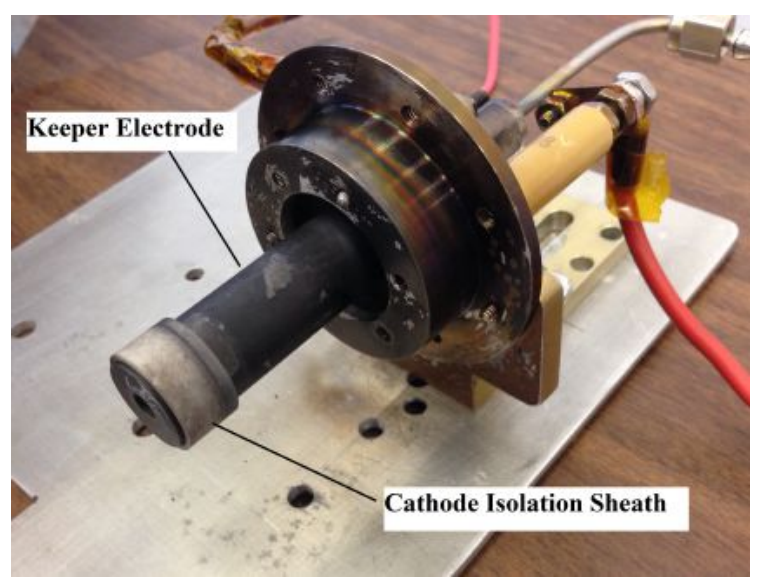

Figure 2.9: The cathode isolation sheath in its intended location at the end of the keeper electrode.

\subsubsection{The Plasma Confinement Magnets}

In order to facilitate plasma confinement, MiXI contains three banks of permanent magnets located throughout the thruster. The banks are placed in a ring configuration; the upper and middle rings wrap around the exterior wall of the anode, while the lower ring is located toward the thrusters base, and forms a surrounds the hollow cathode's keeper electrode. The upper and middle rings are sandwiched between the anode and the anode pole piece; each of these rings contains 30 stacks of $31 \mathrm{mmx} 3 \mathrm{~mm}$ samarium cobalt (SmCo) magnets. The lower ring consists of 20 stacks of $41 \mathrm{mmx} 3 \mathrm{~mm}$ SmCo magnets. As stated in Chapter 1, the magnets are the first part of the thruster to overheat at about $300 \mathrm{An}$ image illustrating the location of each ring is shown in fig. 2.10[15]. 


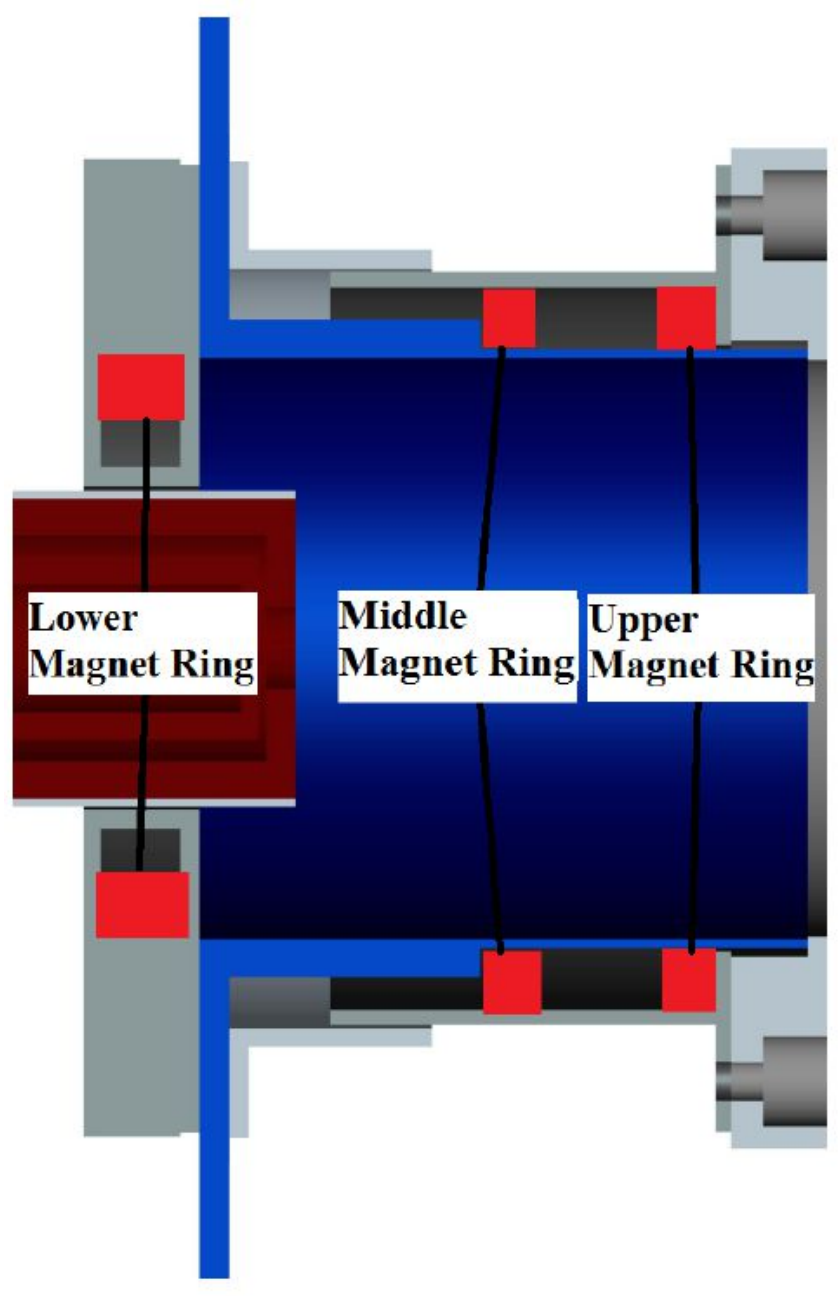

Figure 2.10: A 2D cutaway view of MiXI reveals the location of the three rings of magnets (magnet size is not to scale).

The images in fig. 2.11 provide a three-dimensional view of the magnet ring locations. 


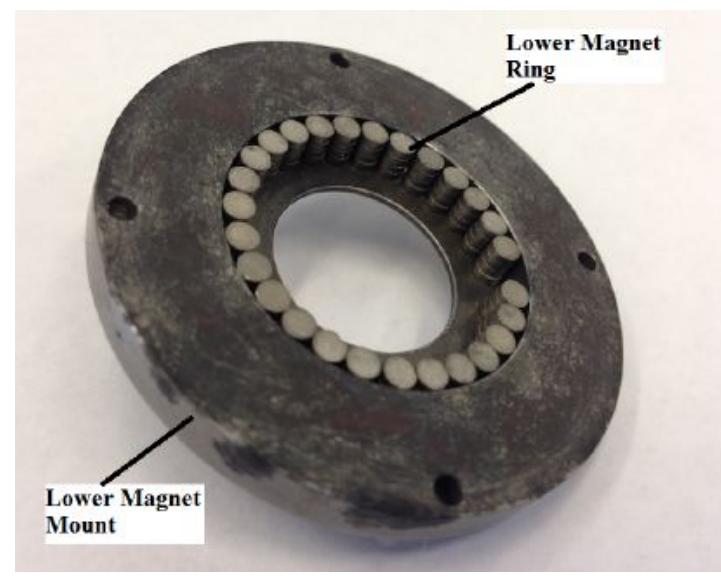

(a) The lower magnet mount with lower ring of plasma confinement magnets.

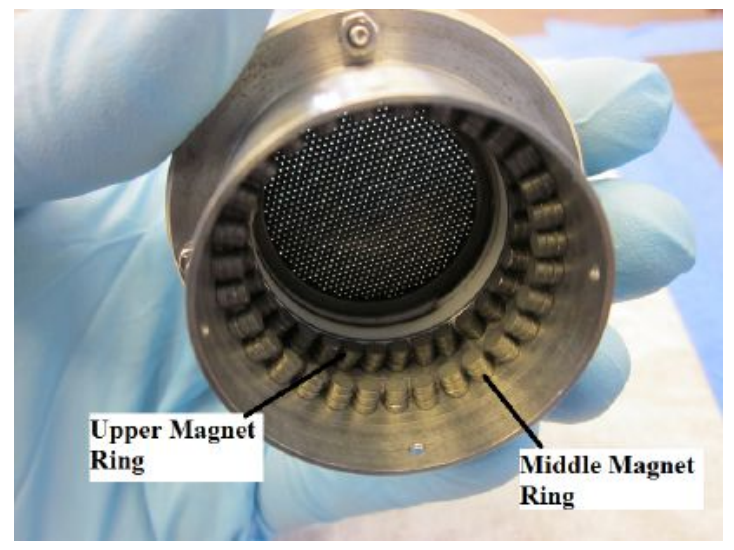

(b) The anode pole piece with the middle and upper rings of plasma confinement magnets

Figure 2.11: This image shows the location of the plasma confinement magnets within MiXI.

\subsubsection{The Screen and Accelerator Grids}

The screen and accelerator grids were created by JPL and donated to Cal Poly for the development of MiXI-CP-V1[27]; these grids are shown in fig. 2.12. The grids are made from molybdenum and are $50 \mathrm{~mm}$ in diameter and $0.27 \mathrm{~mm}$ thick. Each grid has a circular aperture area of $706.8 \mathrm{~mm}_{2}$ and a diameter of $30 \mathrm{~mm}$. Each grid contains a total of 892 apertures but the size of the individual aperture holes is 
different on each screen. The screen grid (the interior grid) has $0.38 \mathrm{~mm}$ diameter aperture holes and the accel grid (the exterior grid) has $0.19 \mathrm{~mm}$ aperture holes. Proper alignment of the holes crucial to the performance of the thruster, therefore each grid has two small holes just outside the aperture area that can be aligned using a hex wrench[15].

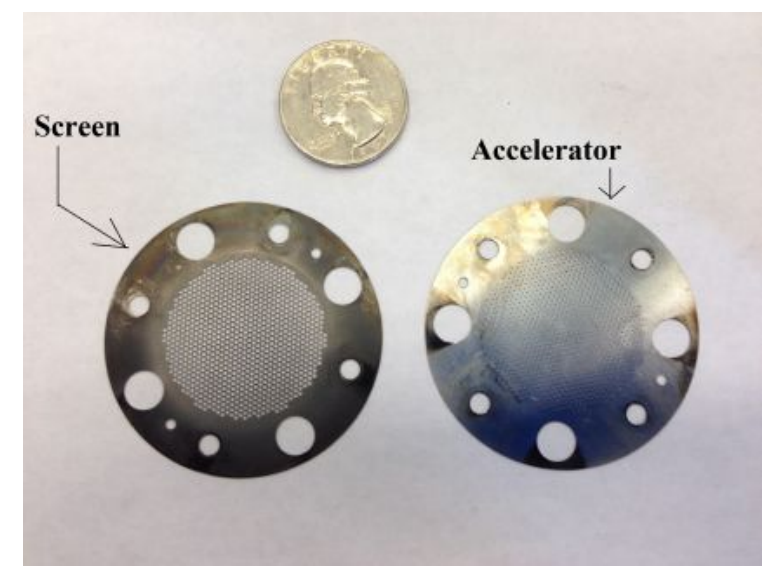

Figure 2.12: MiXI's Screen and Accelerator grids.

The screen and accel grids were designed to have a gap of $0.3 \mathrm{~mm}$ between them. However, wear and tear from testing over the years has left the grids slightly warped, creating gaps of $<0.3 \mathrm{~mm}$ in some places. Past operators of MiXI believed that this led to arcing and electrical breakdown across the gap. Therefore, MiXI-CP-V3 has a gap of $0.6 \mathrm{~mm}$ between the grids, which is accomplished by placing three $0.2 \mathrm{~mm}$ washers between the grid isolation mount and the accel grid. This is illustrated in fig. 2.13.[15, 27] 


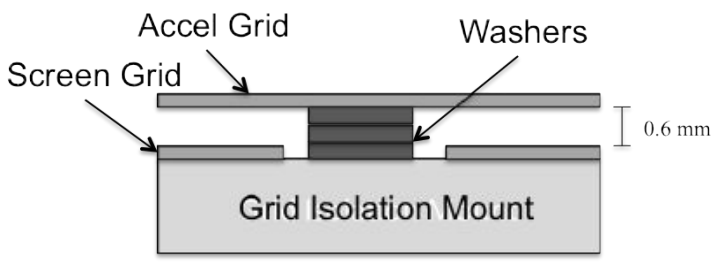

Figure 2.13: Placement of Screen and Accel Grids.[15]

\subsection{Final Assembly}

Once the magnets and grids are in place, the next step is to assemble MiXI and integrate it with the hollow cathode. The anode pole-piece assembly is attached to the anode's flange via the anode cap piece. The anode cap piece, the shield, and the lower magnet mount are all fastened to the anode's flange by four screws. Once this was complete, the MiXI subassembly could be integrated with the hollow cathode. An exploded view of the final configuration is shown in fig. 2.14. 


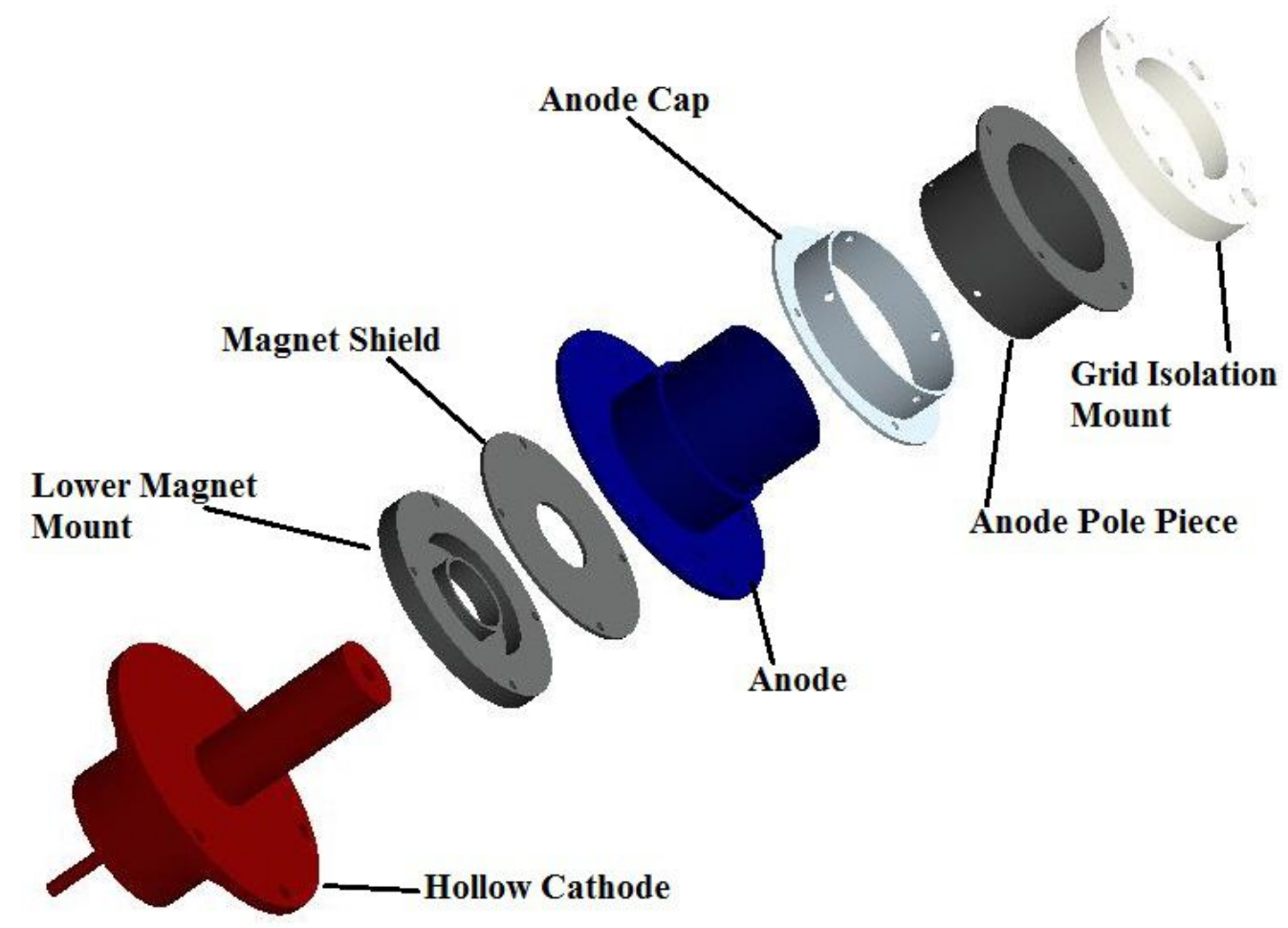

Figure 2.14: Exploded view of MiXI. Not shown are the grids and fasteners.

For this particular experiment, the hollow cathode was integrated with MiXI slightly differently than it was for Mr. Knapp's original design. Figure 2.15a shows the MiXI-cathode configuration as Mr. Knapp had designed it. This configuration had MiXI attached to the cathode flange via four screws and ceramic isolators. Since Mr. Knapp completed the testing for his thesis, the cathode configuration has changed and the current cathode has a different chassis. The mounting locations on this chassis did not match up to the mounting locations on MiXI's anode, so a new integration method was devised. Rather than mounting the anode's flange directly to the cathode's chassis, a stand for MiXI was created out of a $1 / 8$ " sheet of aluminum. The stand is essentially an L-bracket, with a U-shape cut into one of the surfaces so that the keeper electrode could be inserted 
into MiXI's discharge chamber. Attached to the bottom surface of the L-bracket is a sheet of Macor to electrically isolate the bracket from the test stand within the vacuum chamber. This configuration is shown in fig. 2.15b.

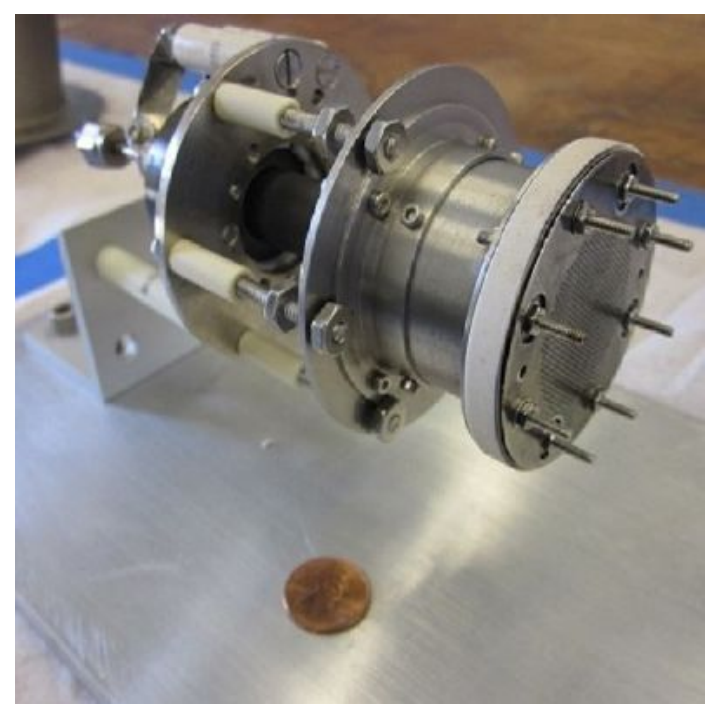

(a) The original configuration as designed by David Knapp.[15]

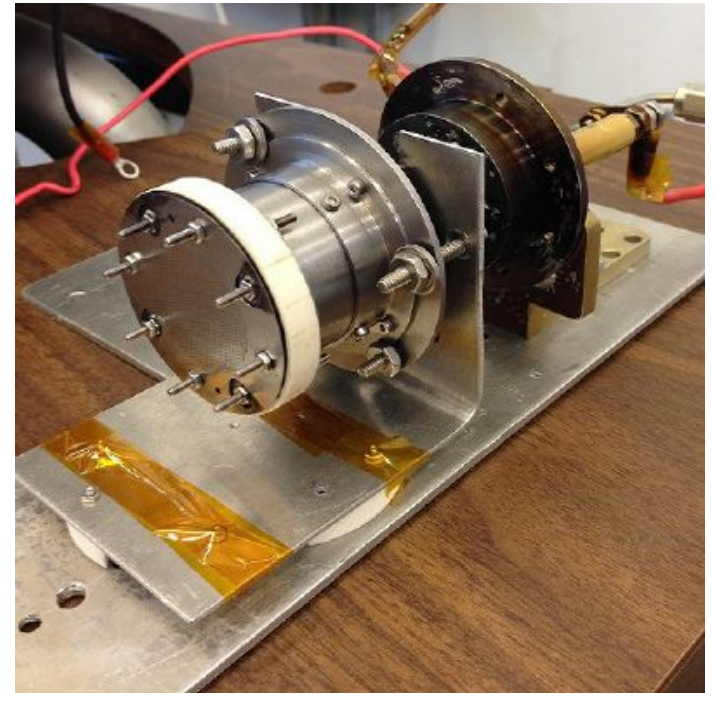

(b) The current configuration.

Figure 2.15: This image shows the two MiXI-Hollow Cathode configurations that have been used for MiXI-CP-V3.

The magnetic field topography that was determined by Mr. Knapp dictates that the keeper electrode must be inserted $8 \mathrm{~mm}$ into the discharge chamber. The 
cathode isolation sheath separates the keeper electrode from the rest of MiXI, and if placed properly, should not sit flush with the back wall of MiXI.[15] 


\section{Testing}

Thermal testing of MiXI was conducted for two reasons: to determine the time rate of temperature change of the thrusters heat sources, and to validate the accuracy of the thermal model. The data for time rate of temperature change was later used as a boundary condition in the thermal model. All thermal tests were performed in Cal Poly's Space Environments Laboratory. This chapter discusses the testing facilities and procedures that were used for testing.

\subsection{Apparatus}

\subsubsection{Vacuum Chamber}

Validation testing for this project utilized the Minimum Atmospheric eXperimentation (MAX) chamber in the Space Environments Laboratory at Cal Poly, San Luis Obispo. MAX, shown in fig. 3.1 and fig. 3.2, has a 12 inch tall, 18 inch diameter cylindrical jar that encloses the test section. The MAX chamber utilizes a Varian Scroll Vacuum Pump to bring the pressure within the chamber

down to about $8 \times 10^{-3}$ Torr. The chamber pressure is further reduced using a CTI CryoTorr 10 cryo pump, which utilizes a CTI-Cryogenics 1020R compressor. With the help of the cryopump, the chamber pressure got as low as $5.2 \times 10^{-7}$ Torr. Pressure within the chamber was determined with two convectron gauges and a Granville-Phillips ion gauge. The convectron gauges were can measure 
pressures as low as $1 \times 10^{-3}$ Torr and are only used for roughing out the chamber or the cryo pump. Once the cryo pump reduces the chamber pressure below $1 \mathrm{x}$ $10^{-3}$, the ion gauge is turned on to obtain an accurate pressure reading. This ion gauge is calibrated to measure the pressure of nitrogen, so when the xenon gas is introduced into the chamber a correction factor of 2.87 must be multiplied by the indicated pressure to acquire an accurate pressure reading.

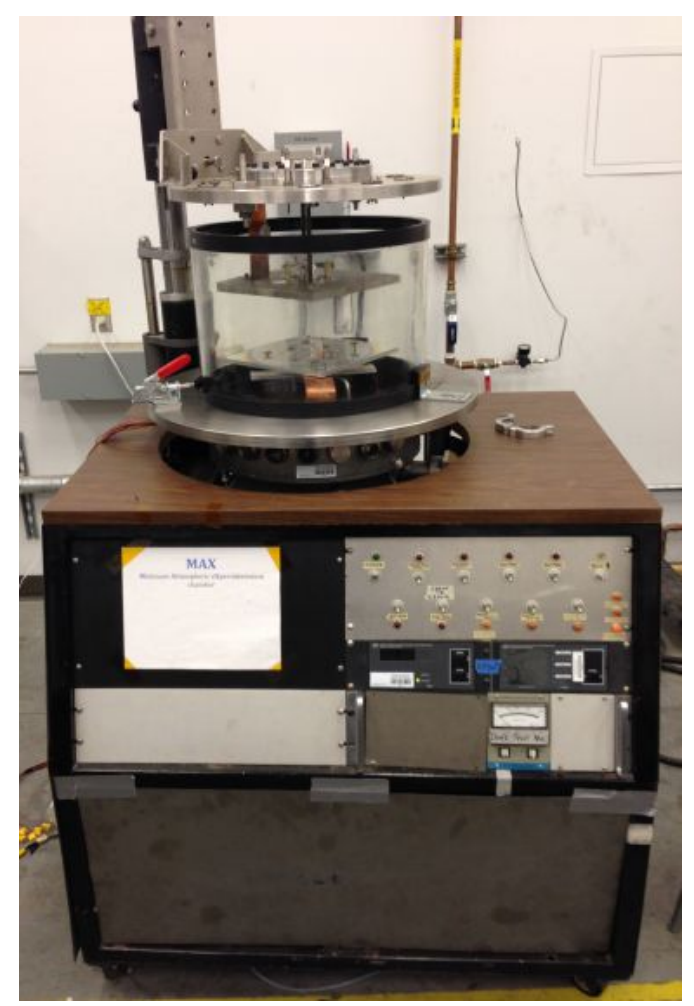

Figure 3.1: Minimum Atmospheric eXperimentation (MAX) Chamber in Cal Poly's Spacecraft Environments Laboratory. 


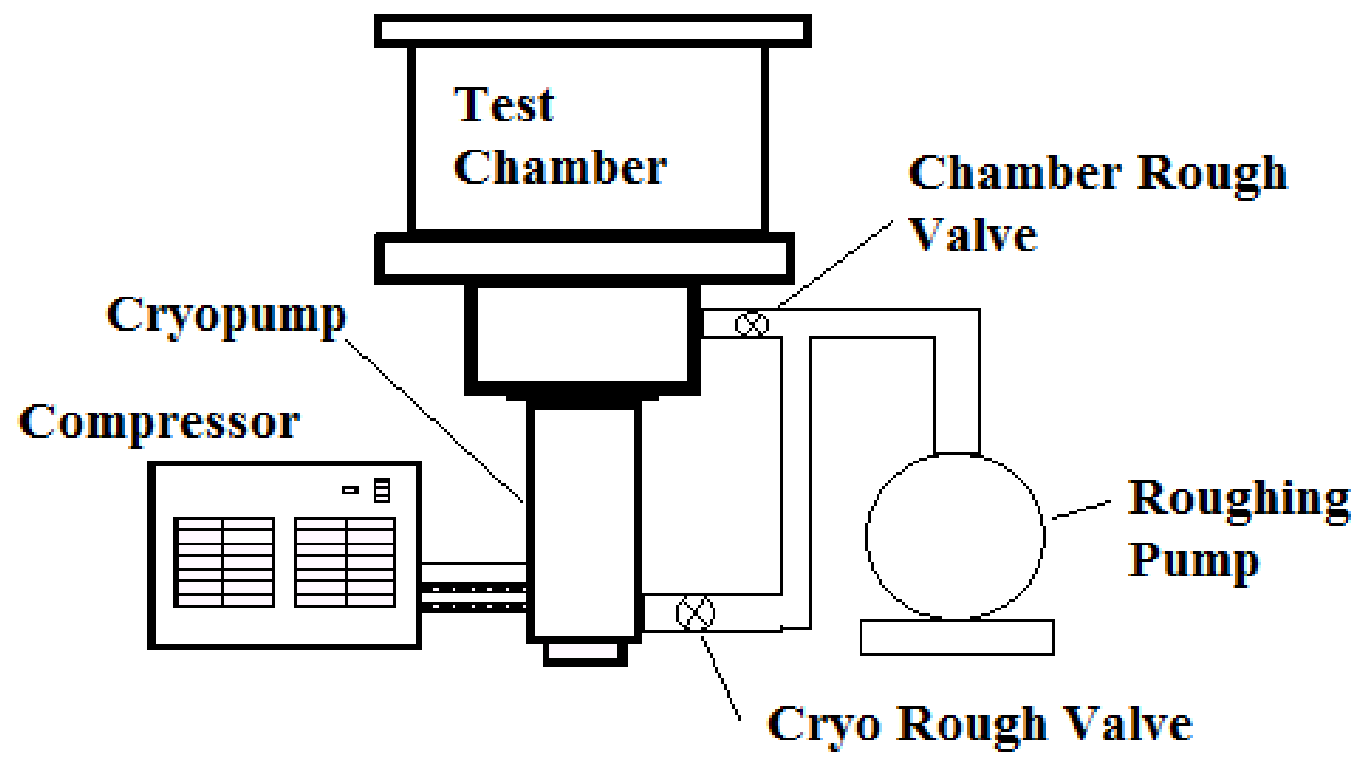

Figure 3.2: A schematic of the MAX vacuum chamber apparatus in the Cal Poly Space Environments Laboratory.

The MAX chamber has 26 feed-through locations. Of these 26, eight were utilized for this experiment and the remaining ports were sealed off. Six ports were used for the electrical system, one was used to feed propellant to the thruster, and one was used for thermocouples. MiXI's hollow cathode and discharge chamber were supplied with electricity through four isolated conductive-rod electrical feedthroughs on the lid of the chamber (one for the keeper, heater, discharge chamber, and negative terminal). Two BNC feedthroughs were used to power the accel grid and the CTFC neutralizer.

\subsubsection{Electrical System}

The electrical system is one of the most complex parts of any ion thruster. Even a small lab thruster like MiXI requires a minimum of six power supplies. MiXI's hollow cathode requires two power supplies, one for the heater and one for the 
keeper. The heater is used for raising the temperature of the cathode's insert to the temperatures required for electron emission. Once the hollow cathode is hot enough, the keeper is activated and initiates electron emission during startup in addition to reducing the ion bombardment energy during nominal operations. After 'striking' the hollow cathode discharge, the heater's power supply may be turned off; at this point, the heat from the plasma is enough to sustain further plasma generation.

The discharge chamber (also known as the anode) requires it's own power supply. The discharge supply applies a positive voltage to the anode that will attract electrons from the cathode and from the inside of the discharge chamber. The screen supply is connected between the anode and the common node of the neutralizer and accel supplies. The neutralizer cathode consists of a CTFT and is used to render the thruster's plume quasi-neutral (equal electron and ion densities). The accel power supply negatively biases the accel grid, which prevents electrons in the plume from entering the discharge chamber.

MiXI's electrical system requires two circuits in order to supply the grids with the proper voltage. The first circuit consists of the keeper supply, heater supply, discharge supply, and the igniter supply. This circuit is kept at a "floating" ground and must be isolated from the common ground at all times. The second circuit consists of the screen supply, accel supply, and neutralizer supply and is grounded to the common ground. These circuits can be seen in fig 3.3. 


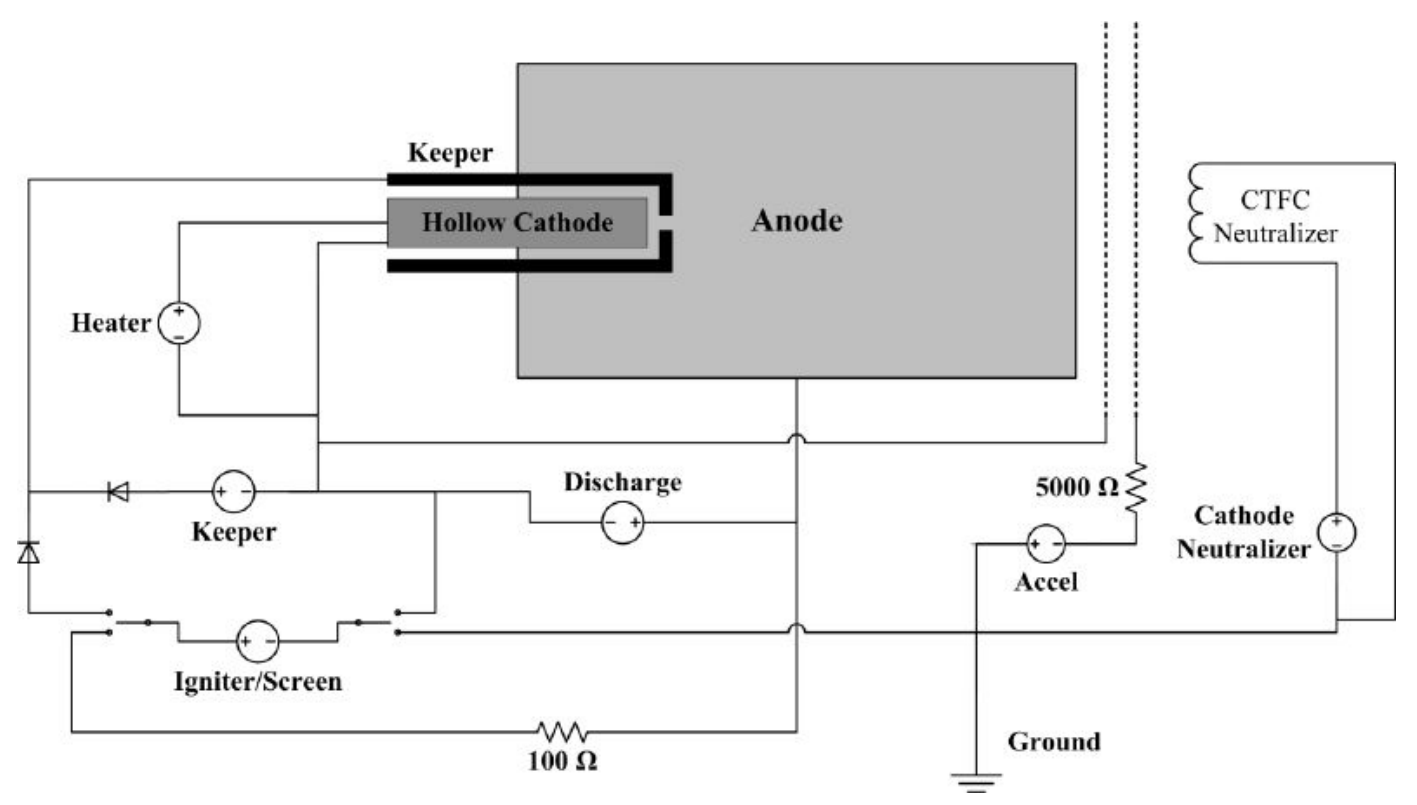

Figure 3.3: Circuit diagram of MiXI's elelctrical system.[15]

When striking the hollow cathode, the keeper may require up to $1 \mathrm{kV}$. This is more than a typical medium voltage, medium-current power supply can provide; therefore, it is necessary to use the high voltage, low current power supply to initially strike the cathode. The power supply that is normally used for the screen was used to ignite the cathode then turned off and switched to the screen circuit. It can be seen from fig 3.3 that diodes were placed in between the keeper and igniter power supplies. This was necessary to prevent one of the power supplies from damaging the other. It should also be noted that the potential difference between the two grids sometimes caused arcing. In order to limit the amount of current that results from this arcing, a resistor was placed in between each grid and the grids respective power supply. 


\subsubsection{Propellant System}

The propellant system used during testing consisted of a propellant system isolator, a digital flow meter, a needle valve, a high purity regulator, and a tank of xenon gas. The propellant isolator is necessary to electrically isolate the plumbing of the propellant system and from the hollow cathode. The regulator is intended to be used for high purity gases, which is necessary to ensure that no containments are introduced to the hollow cathode. A needle valve was used to control the flow rate of the propellant and a digital flow meter was needed in order to measure the actual flow rate. It should be noted that he flow meter is calibrated for nitrogen so a correction factor of 1.44 was applied to the indicated flow rate. Past versions of MiXI used a Swagelok diaphragm valve to shutoff the propellant flow at the end of testing; because the regulator has a built-in shutoff valve, the diaphragm valve was deemed superfluous and left out of this setup. The xenon gas was acquired just prior to the commencement of testing and was ultra-high

purity grade, or $99.999 \%$ pure xenon. For a detailed list of propellant system hardware, see the procedures in appendix D. 


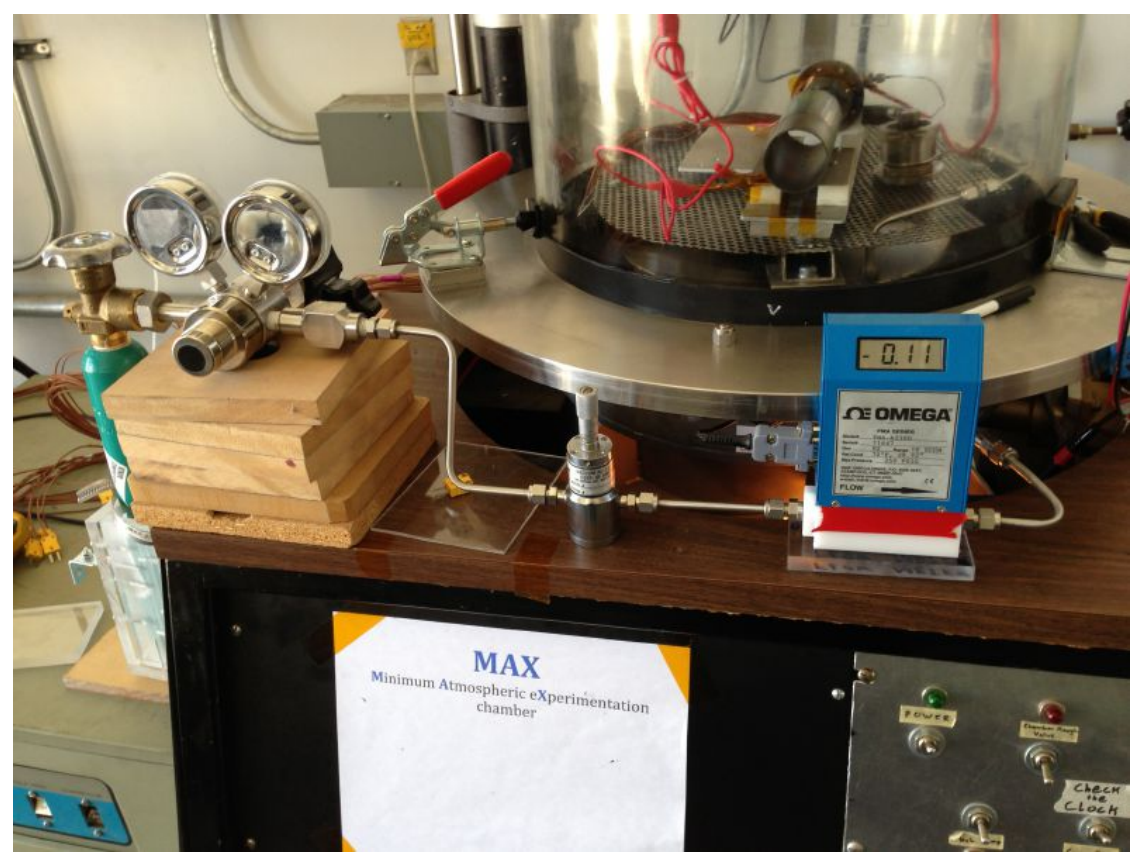

Figure 3.4: The portion of the propellant system that remains outside of the vacuum chamber.

\subsubsection{Data Acquisition}

Temperature was measured at eight locations on the outside of the anode pole piece. In order to acquire the thermocouple data, a National Instruments cDAQ9174 chassis was used in conjunction with two NI 9211 thermocouple measurement devices, shown in figures 3.5 and 3.6 respectively. Eight Omega type K thermocouples were used to measure the temperature just outside MiXI. 


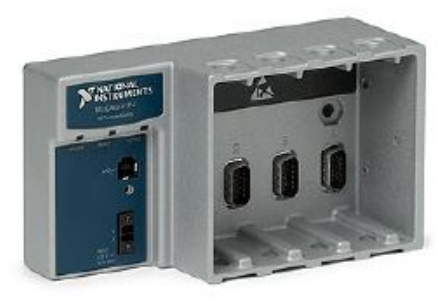

Figure 3.5: The NI Data Acquisition Chassis that was used for testing[4].

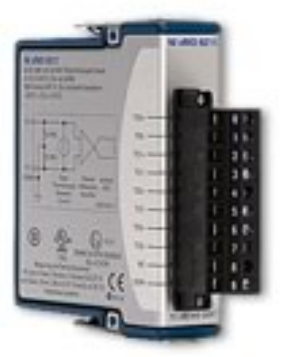

Figure 3.6: One of the two NI 9211 Thermocouple Input Module that was used for testing[4].

There were only two thermocouple measurement DAQ's available for testing. Each 9211 DAQ could collect data from four thermocouples, therefore, temperature data could only be measured from eight locations. The thermocouples were all located in the same two dimensional plane with two on the top of MiXI's polepice, two on the cap, one on the flange of the anode, and three on the outside of the magnet-mount. Placing thermocouples in the same 2D plane was appropriate because the thermal model being validated is two dimensional. Because MiXI is symmetric about its center axis, it was assumed that the temperature distribution was uniform throughout the thruster; therefore, only half of MiXI needed to be modeled. Rather than arbitrarily scattering thermocouples throughout the 
thruster, they were concentrated in areas that were being modeled.

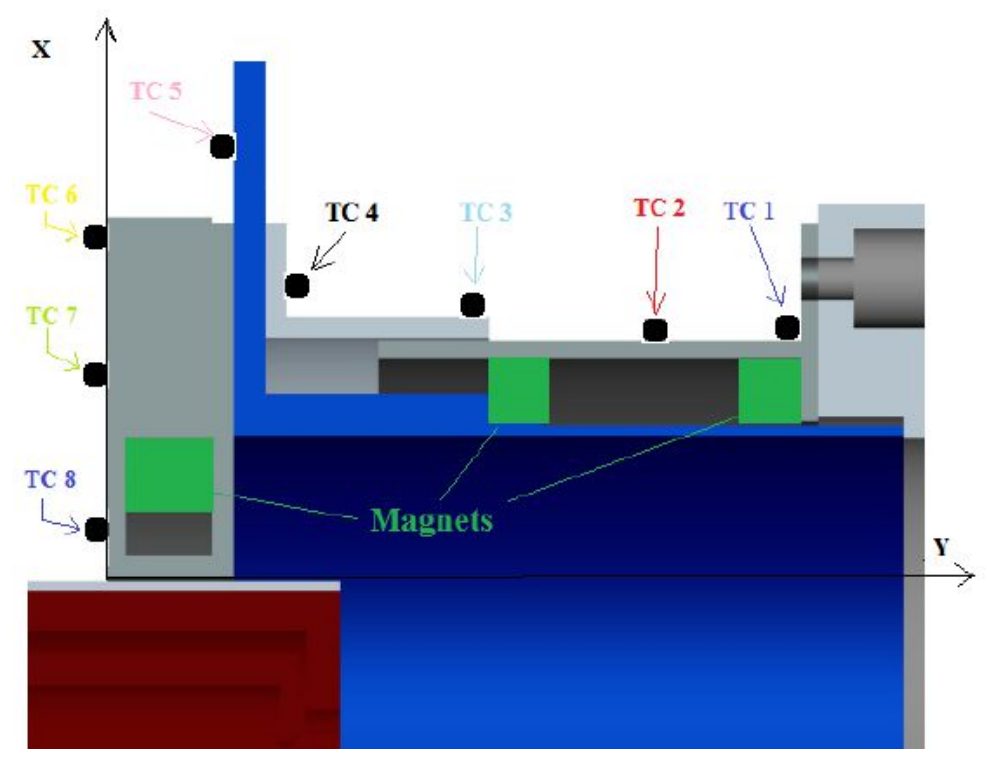

Figure 3.7: This figure shows the location of each thermocouple that was used to collect temperature data during MiXI's operations.

The precise location of the thermocouples is shown in table 3.1. The initial plan was to place the thermocouples at even intervals, starting at the grids and moving towards the back of the thruster. Practical limitations encountered during testing prevented this so some of the thermocouples had to be relocated. For example, thermocouple 1 ( $\mathrm{TC} 1$ ) was initially to be located on top of the grid isolation mount, just next to the grids. When the time came to attach the thermocouples to the thruster, TC 1 could not be fixed to the isolation mount without interfering with the screen and acceleator grids. Therefore, it was placed at the corner of the pole-piece flange, as seen in fig. 3.7. Thermocouples 2, 3, and 4 were spaced $7 \mathrm{~mm}$ apart in the $\mathrm{x}$-direction. Similarly, thermocouples 6 , 7, and 8 were placed $7 \mathrm{~mm}$ apart in the y-direction. Thermocouple 5's location was selected because it was desired to have a thermocouple on the anode flange; therefore, TC 5 was located in the center of the exposed portion of the anode's 
flange. The thermocouples were secured to the surface of the thruster with kapton tape; in the future, however, it may be desirable to weld the thermocouples to the surface. Small welds would be removable after the completion of the tests and should not cause permanent harm to the thermocouples.

Table 3.1: Locations of the thermocouples that were used to record temperature data.

\begin{tabular}{|c|c|c|}
\hline Thermocouple & $\mathbf{X}(\mathbf{m m})$ & $\mathbf{Y}(\mathbf{m m})$ \\
\hline TC 1 & 33 & 12 \\
\hline TC 2 & 22 & 12 \\
\hline TC 3 & 15 & 13 \\
\hline TC 4 & 8 & 15 \\
\hline TC 5 & 6 & 20 \\
\hline TC 6 & 0 & 16 \\
\hline TC 7 & 0 & 9 \\
\hline TC 8 & 0 & 2 \\
\hline
\end{tabular}

In order to monitor and store the thermocouple data, a virtual instrument (VI) was created using National Instrument's LabView program. The VI was set to continuously take samples at a frequency of 1 sample per second. The primary purpose of the VI was to record thermocouple data during thruster operation but it was useful for monitoring the chamber's thermal environment in between tests. For this reason, the VI was equipped with a 'record' button so that the VI could be used without constantly recording data.

\subsection{Procedure}

This section summarizes the testing procedure. For the complete vacuum chamber, hollow cathode, and MiXI procedures, see Appendix D.

Preparations for testing began days before the actual test was performed. 
The vacuum chamber was run through at least one regeneration cycle prior to the test. As part of a regeneration cycle, the cryo chamber is cooled down to about 15 to 20 Kelvin. Then the compressor is shut off and the cryo chamber is allowed to warm up with the mechanical pump is still operating. This removes any contaminants that that have entered the cryo by pumping them out of the chamber as they change phase from solid to gas. After regeneration was complete, the cryo chamber was pumped down once again.

While the compressor was cooling the cryo, the main vacuum chamber and all components of MiXI were thoroughly cleaned with acetone to remove all contaminants. From that point forward, anything that was placed in the vacuum chamber was handled with powder free gloves to prevent fingerprints and other foreign matter from contaminating the system. MiXI and the Hollow Cathode were carefully integrated and placed in the vacuum chamber. At this point, all of the thermocouples were attached to the outside of the thruster according to the diagram in 3.7. The thermocouples were held to the surface with Kapton tape. Then, the thruster and hollow cathode were integrated with the propellant and power supplies. The electrical connections were carefully checked and rechecked using a digital multimeter. Once the electrical connections were determined to be correct, the vacuum chamber was closed and pumped down with the roughing pump. When the chamber pressure had descended below 10 miliTorr, the gate valve was open, combining the cryo chamber and the testing section of the vacuum chamber. Testing began when the pressure inside the chamber dropped below 1 microTorr. 


\subsubsection{MiXI Operation}

For these tests it was very important to acquire consistent temperature data; therefore, the exact same procedure was followed for each test. The power supply that controls the hollow cathode's heater was turned on and set to 6 Amperes. Once the heater temperature reached 6 Amps, the 'Record' button on the LabView VI was switched on and the recording of thermocouple data began. The cathode was allowed to heat up for exactly 15 minutes at which point, the xenon tank and high purity regulator were opened and the flow rate was set to $7 \mathrm{sccm}$ using the needle valve. The voltage on the hollow cathode's keeper supply was increased until plasma began to form; at this point, the keeper power supply

switches to current control and the keeper current is adjusted to 1.75 Amps. The heater supply is turned off while the discharge current is adjusted to 3.2 Amps. At this point, the propellant supply was reduced to $1 \mathrm{sccm}$ and, the neutralizer was switched on and the screen and accel grids were biased to -100 and 750 Volts respectively.

To ensure that the data was consistent, data was collected at the same operating conditions for each test. The conditions chosen for operation are those that correspond with the highest thrust, specific impulse (Isp), and mass utilization efficiency. The conditions can be seen in table 3.2. 
Table 3.2: Thruster operating conditions.

\begin{tabular}{|c|c|c|}
\hline Power Supply & Voltage (V) & Current (A) \\
\hline Heater & 15 & 6 \\
\hline Keeper & 20 & 1.75 \\
\hline Igniter & 1000 & 0.125 \\
\hline Discharge & 30 & 6 \\
\hline Screen & -100 & 0.035 \\
\hline Accel & 750 & 0.100 \\
\hline Neutralizer & 16 & 5.5 \\
\hline
\end{tabular}

The thruster was allowed to operate for six minutes after the cathode had been struck. At this point, some of the thermocouples indicated that the temperature on the anode had reached $300^{\circ} \mathrm{C}$. This is the 'never exceed temperature' of the permanent magnets inside MiXI. To avoid damaging the magnets, the thruster was powered down by turning off each power supply in the reverse order from which they were turned on; the needle valve on the propellant line was closed to stop propellant flow. LabView was allowed to continue recording data for an additional 10 minutes after the power supplies were turned off as the thruster began to cool down. Once Data recording had ceased the thruster was allowed to continue cooling under vacuum until it reached $30^{\circ} \mathrm{C}$, and a new test could commence.

Once enough data had been collected the thruster was allowed to cool to $30^{\circ} \mathrm{C}$, and experimental closeout could begin. The gate valve on the vacuum chamber was closed and the compressor was turned off. The portion of the chamber that contained the experiment was vented and equalized to ambient pressure. Then, the chamber was opened and MiXi was removed along with all of the electrical and propellant system components. MiXI and the hollow cathode were carefully examined for any signs of damage that may have occurred during testing. MiXI 
and the hollow cathode were carefully packaged and returned to storage.

\subsection{Data Reduction}

Thermocouple data was collected for a total of 11 tests, one of which was deemed to be an out-lier and had to be thrown out. Figure 3.8 shows the raw data from all 11 tests superimposed over one another. (It should be noted that the line-plots presented in this report were created from a set of discretized data collected at a rate of one data point per second. Line plots were used in lieu of scatter plots for aesthetic purposes.) The first 15 minutes of data was recorded while the keeper was heating up. Fifteen minutes after data recording had begun, the propellant flow was initiated and the attempt to strike the keeper was begun. Typically, it took about 80 to 90 seconds strike the keeper once the cathode's propellant supply had been initiated. During the first test, the keeper took about five minutes to strike; this is why the thermocouple data from the first test is offset from the data of the other tests. Once the keeper had been struck, the hollow cathode's heater was turned off and the only source of heat came from the plasma within the thruster's discharge chamber. This phase of operations took place between 16.5 and 22.5 minutes; 22.5 minutes into data collection, the magnets nearest thermocouple 8 were close to their maximum operating temperature of $300^{\circ} \mathrm{C}$. 


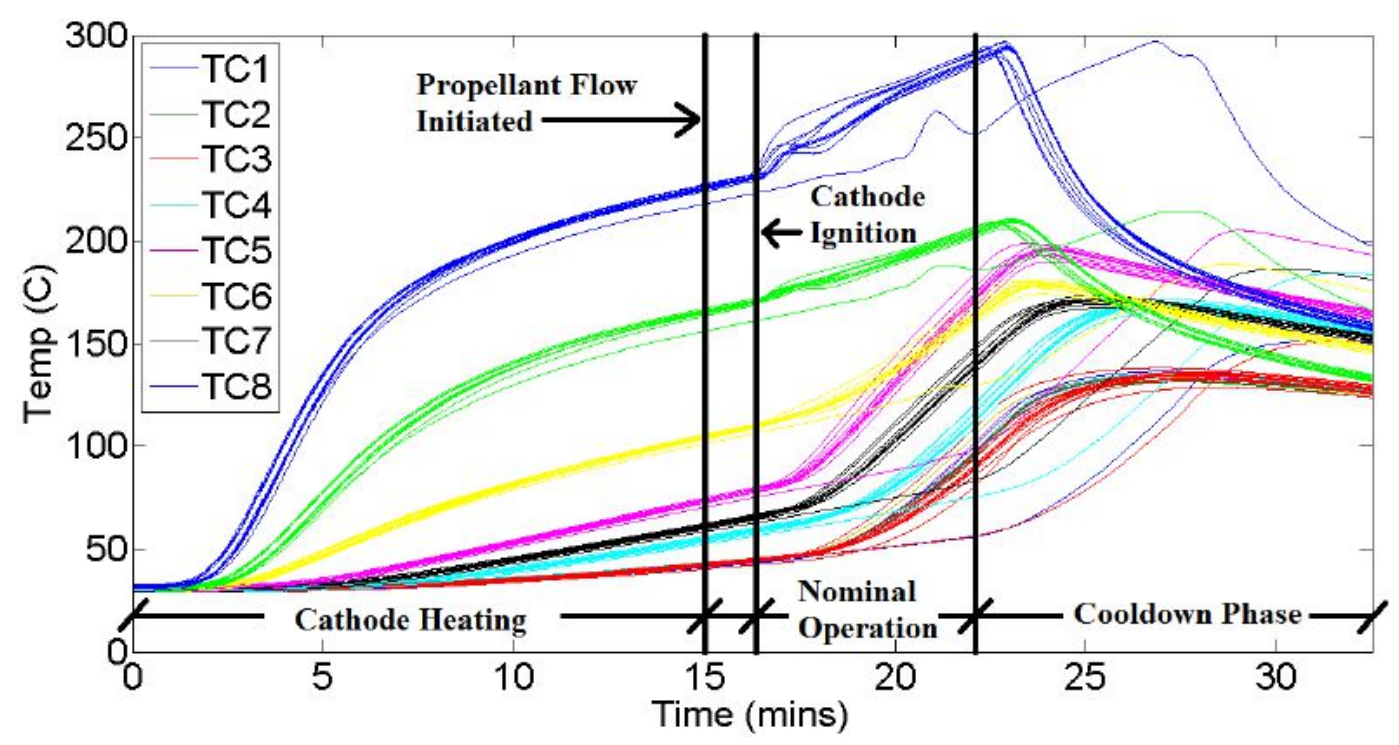

Figure 3.8: All of the data that was collected during the 11 tests. The data from the first test is shifted to the right of all subsequent tests because it took several minutes longer for the keeper to strike. Therefore, data from this test was considered to be an out-lier and thrown out of final calculations. This data has been smoothed using MATLAB's built-in 'smooth' function.

The reason for the five minute delay during the first test is difficult to explain. The hollow cathode used for MiXI-CP-V3 can be described as fickle and temperamental. The ignition process for this cathode involves introducing propellant into the cathode, and increasing the voltage of the keeper electrode until the keeper insert emits enough electrons to ionize the propellant. During this portion of the project, it was observed that the time it takes the insert to emit the electrons necessary to ionize the propellant can vary from test to test. However, this ignition time becomes relatively short if the cathode is operated on a regular basis. When testing for this project began, this cathode had not been operated since David Knapp's tests over a year earlier. The lack of use prior to this project meant that cathode ignition was difficult to achieve, hence the five minute delay in cathode ignition during the first test. 
During the six minutes between the cathode being struck and the power supplies being powered down, the test data shows small, erratic spikes in temperature from thermocouples 5 through 8 . These spikes can be seen in fig. 3.9. During this portion of the test there was minor arcing throughout the chamber. Most of the arcing was observed to be occuring between the screen and accel grids, and between the keeper electrode and MiXI's lower magnet mount. This arcing is the result of the high potential difference between the grids, and between the keeper electrode and MiXI. Thermocouples 5-8 were effected by the arcing between the keeper and MiXI because those thermocouples were closest to the keeper. As fig. 3.7 illustrates, thermocouples 5-8 were located on a plane perpendicular to the keeper electrode, exposing them to arcing from the keeper. All of this arcing was detected by the thermocouples and resulted in erroneous temperature data. The apparent temperature spikes caused by the arcing was smoothed out using MATLAB's built-in 'smooth' function.

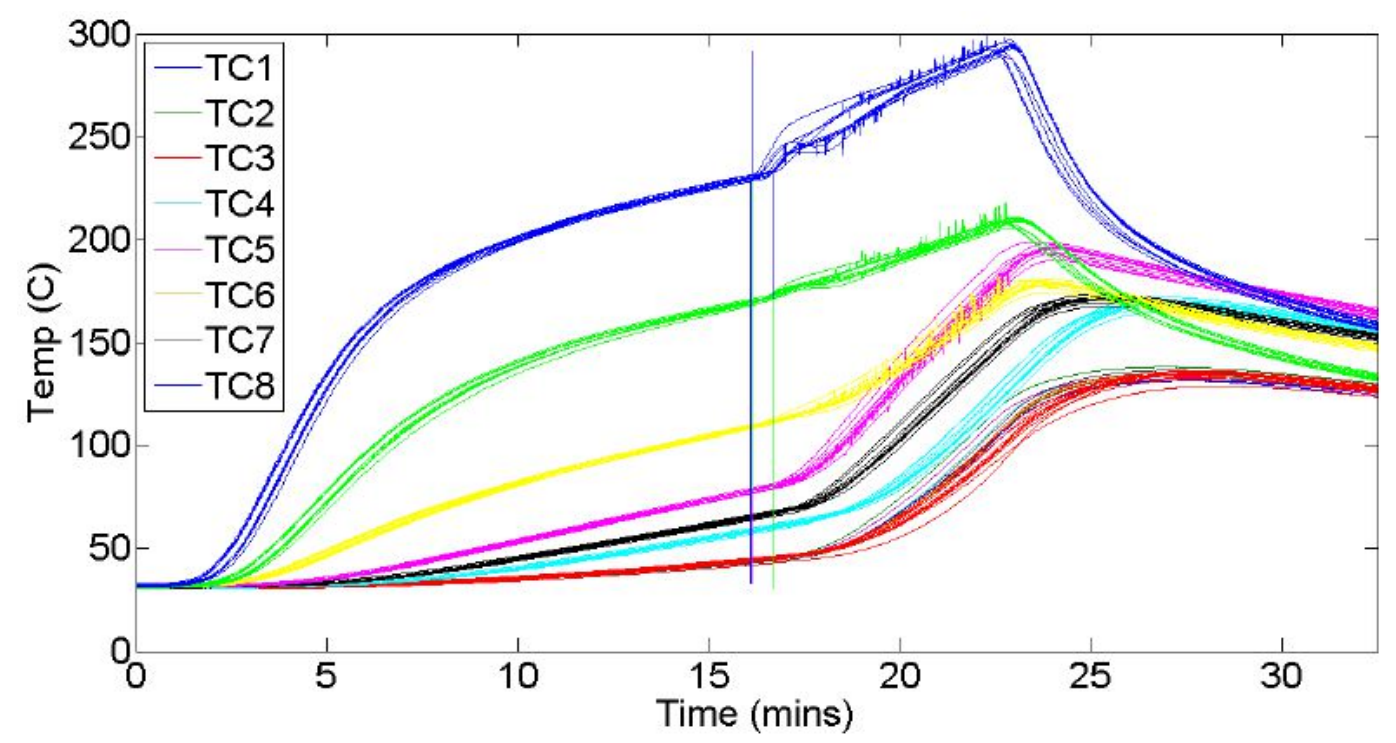

Figure 3.9: Original data from tests 2-11 before it had been smoothed. Notice the small spikes in thermocouples $5-8$ between 16 and 23 minutes. These spikes are due to arcing throughout the chamber during operation of the thruster. 
Figure 3.10 shows the smoothed thermocouple data for tests 2 through 11. As stated previously, the data from the first test was omitted because it was more than two standard deviations away from the mean. From this image, it is very clear that the temperature of thermocouples 6,7 , and 8 increase at a much faster rate than any of the other thermocouples. Also, the difference in temperature between these thermocouples at the 16.5 minute mark is notably large considering that the thermocouples are only $7 \mathrm{~mm}$ apart. It is believed that this large temperature gradient is due to radiative heat transfer from the surface of the keeper to the walls of MiXI.

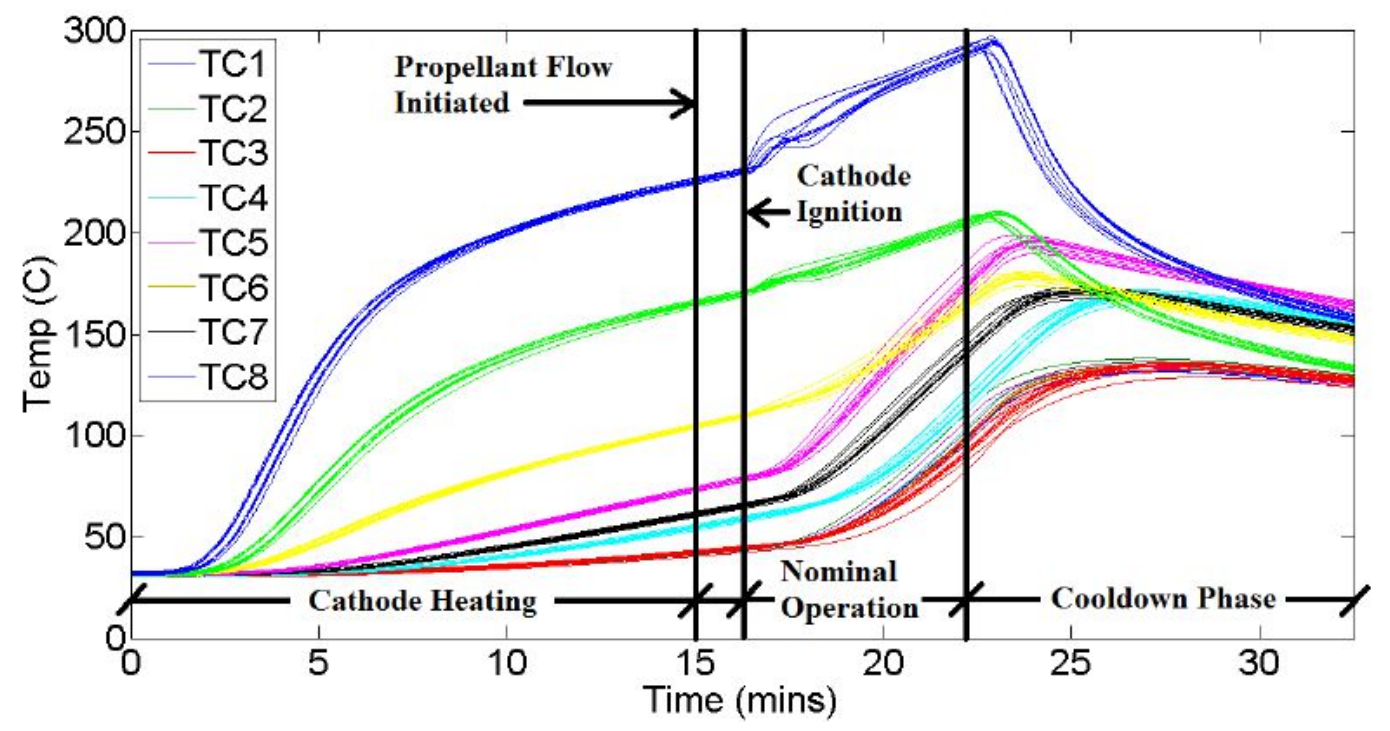

Figure 3.10: The data from tests 2-11 after being smoothed using MATLAB's 'smooth' function.

Figure 3.11 shows the mean of the 10 tests that were considered. This average was determined after the spikes within the data had been smoothed. This is the data set that will be compared to MiXI's thermal model in order to determine its accuracy. This plot indicates the different phases of thruster operations and is shown without error bars to reduce the amount of clutter on the image. For error bars please refer to fig. 3.12. 


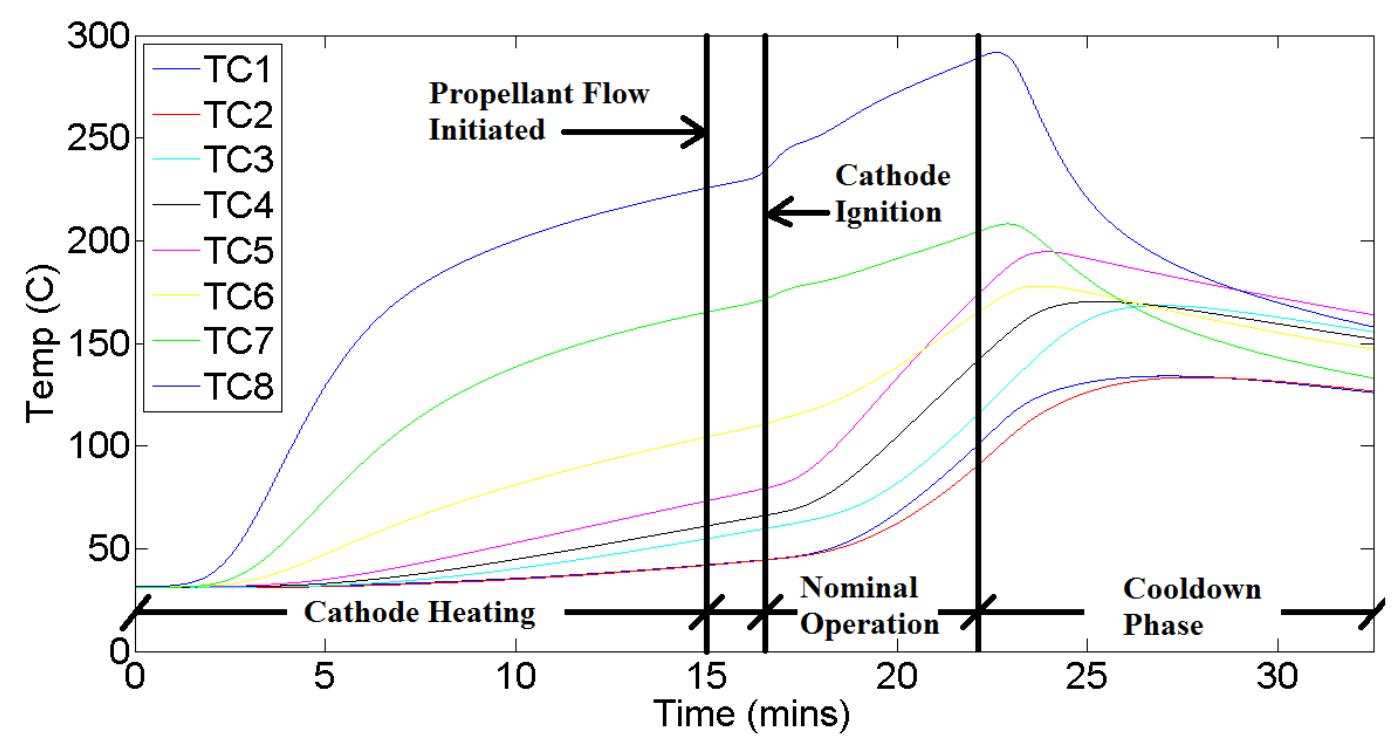

Figure 3.11: The mean of the smoothed data from tests 2-11. This is the data that will be used to validate MiXI's thermal model.

Figure 3.12 shows the average of the 10 tests with error bars to indicate one standard deviation from the mean at that point in time. For thermocouple 8, the standard deviation is highest just after cathode ignition and just after cathode shut down. For all other thermocouples, the standard deviation is highest during the thrusters nominal operation phase (between 16.5 and 23.5 minutes). The reason why the standard deviation is highest for thermocouple 8 after cathode ignition is due to TC8's proximity to the hollow cathode. At the time of cathode ignition, the heater is still on and actively releasing heat. Sometimes the person performing the test forgot to turn off the heater as soon as the cathode was ignited, causing a larger increase in temperature in TC8 then what was seen when the heater was shut down at the proper time. 


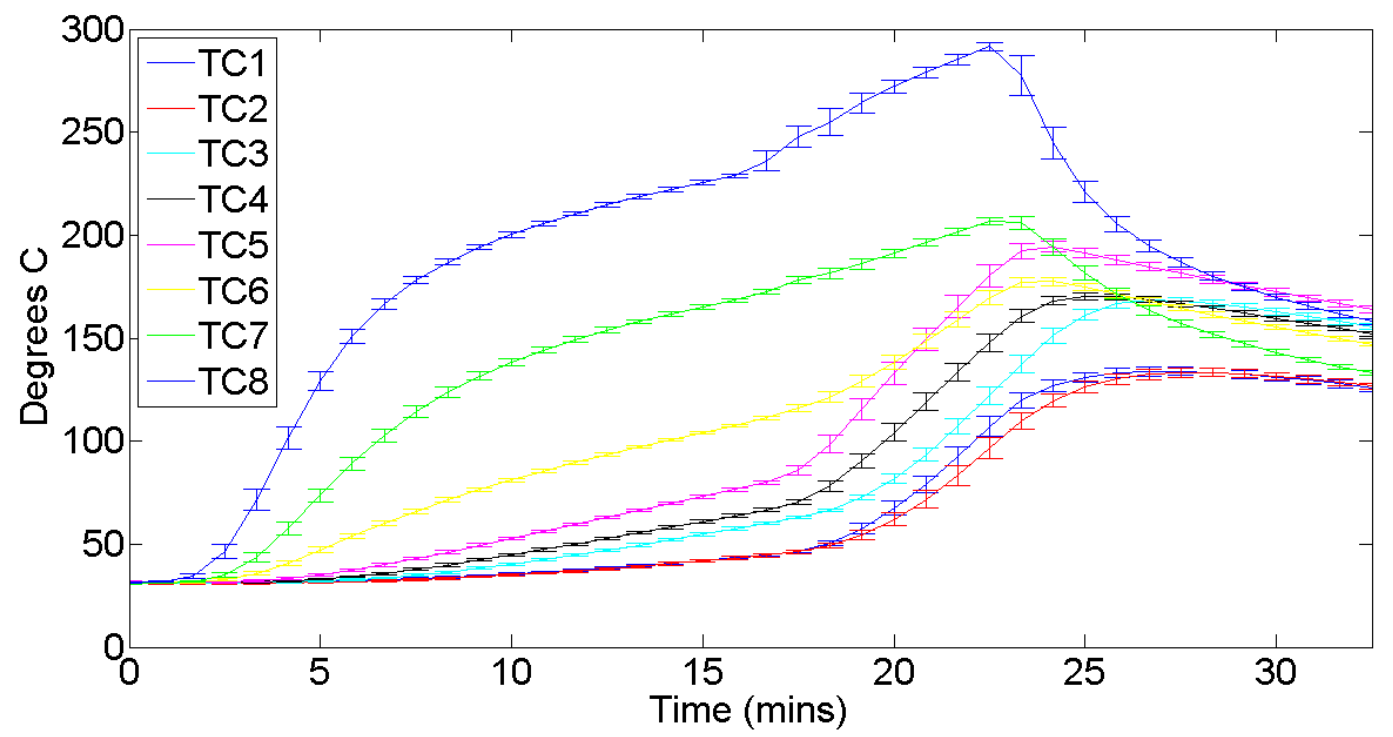

Figure 3.12: The mean of the smoothed data from tests 2-11 with error bars. The error bars indicate one standard deviation from the mean at that point in time. 


\section{Thermal Model}

A model of MiXI and the surrounding vacuum chamber was created to simulate the thermal environment that was observed during testing. The mesh for this model was created in ANSYS ICEM, and then analyzed using ANSYS Fluent. Creating a model for this particular problem was made very difficult by MiXI's complex geometry and the fact that we were modeling a transient temperature profile rather than a steady state profile. This chapter will discuss the software that was used to model MiXI, the thermal model's assumptions, as well as the challenges that were faced and how they were overcome. The chapter will conclude by discussing the final thermal model of MiXI and how it compares to the experimental data.

\subsection{ANSYS Fluent}

The commercially available software, Fluent, by ANSYS was utilized to model the thermal environment of MiXI. Fluent is a computational fluid dynamics software package that uses the finite volume method to numerically solve physical governing equations to model fluid flow and heat transfer. Although Fluent is primarily used to model fluid dynamics, it is capable of accurately modeling all modes of heat transfer (conduction, convection, and radiation).[1, 5]

Fluent was chosen for this project because of its ability to model transient 
conduction and radiation. Additionally, the Aerospace and Mechanical Engineering departments at Cal Poly own many licenses for Fluent, so it was available for the authors use. Although this thermal model ignored fluid flow (this will be discussed in more detail later in the chapter) Fluent's ability to model fluid flow contributed to its selection. The author originally intended to model plasma flow within the discharge chamber with the aid of Fluent's MHD (MagnetoHydroDynamics) add-on module. However, this attempt was abandoned when a license for the MHD module could not be obtained. The author determined that the thesis objectives could still be met while ignoring plasma flow (this will be discussed later in the chapter) and the Fluent model could eventually be re-purposed for modeling plasma should Cal Poly acquire the MHD module; therefore, it was decided to proceed with ANSYS Fluent.[5]

Other software programs that were considered include COMSOL, Thermal Desktop, and Sinda/Fluint. COMSOL was a tempting option because of its ability to model magnetohydrodynamics and heat transfer; however, Cal Poly did not own a COMSOL license at the time this project was begun so it could not be used. Similarly, Sinda/Fluint was ruled out because it is not available for use at Cal Poly due to lack of an active license. As noted by Coleman Younger in his thesis, Thermal Desktop is not capable of modeling small parts or the thin walls of the anode, therefore it was not used for this project[27].

\subsubsection{Governing Equations}

When iterating through a mesh, Fluent, by default, solves the conservation equations for momentum and continuity. When conductive or convective heat transfer is considered, the conservation equation for energy is also solved[5]. Radiative heat transfer is evaluated using one of five radiation models[5]. This section 
presents the governing equations for laminar flow in an inertial reference frame as well as the equations used in the P1 radiation Model.

\section{Conservation of Mass \& Momentum Equations}

This particular thermal model does not consider fluid flow, therefore it is not necessary to evaluate the conservation of mass and momentum equations. However, Fluent is intended to be used to solve fluid dynamics problems and, by default, solves these equations[5]. In order to acknowledge that these equations are being considered by Fluent, they will be briefly discussed in this section.

The conservation of mass equation is as follows,

$$
\frac{\partial \rho}{\partial t}+\nabla \cdot(\rho \vec{v})=S_{m}
$$

where $\rho$ is the density of the fluid, $t$ is time, $\vec{v}$ is velocity, and $S_{m}$ is a mass added by various sources[5].

The momentum equation is solved in the following form,

$$
\frac{\partial}{\partial t}(\rho \vec{v})+\nabla \cdot(\rho \vec{v} \vec{v})=-\nabla p+\nabla \cdot(\bar{\tau})+\rho \vec{g}+\vec{F},
$$

where $p$ is the static pressure, $\rho \vec{g}$ is the gravitational body force, $\vec{F}$ is the external body force, and $\bar{\tau}$ is the stress tensor[5]. The stress tensor is determined by the following equation,

$$
\bar{\tau}=\mu\left[\left(\nabla \vec{v}+\nabla \vec{v}^{T}\right)-\frac{2}{3} \nabla \cdot \vec{v} I\right]
$$

where $\mu$ is the molecular viscosity $I$ is the unit tensor[5]. 


\section{Energy Equation}

When modeling heat transfer, ANSYS Fluent solves the general energy transport equation in the following form,

$$
\frac{\partial}{\partial t}(\rho E)+\nabla \cdot(\vec{v}(\rho E+p))=\nabla \cdot\left(k_{e f f} \nabla T-\sum_{j} h_{j} \vec{J}_{j}+\left(\bar{\tau}_{e f f} \cdot \vec{v}\right)\right)+S_{h}
$$

where $k_{e f f}$ is the effective thermal conductivity, $\vec{J}_{j}$ is the diffusion flux of the species $j, S_{h}$ is the enthalpy source or $\operatorname{sink}[5]$. Finally, energy $(E)$ per unit mass is defined as,

$$
E=h-\frac{p}{\rho}+\frac{v^{2}}{2}
$$

where $h$ is the sensible enthalpy[5]. For incompressible flows, sensible enthalpy is defined as,

$$
h=\int_{T_{r e f}}^{T} c_{p, j} \mathrm{~d} T+\frac{P}{\rho} .
$$

The term $Y_{j}$ in equation 4.6 is the mass fraction of species $j$ and $h_{j}$ is determined by the equation,

$$
h_{j}=\int_{T_{r e f}}^{T} c_{p, j} \mathrm{~d} T
$$

where $T_{\text {ref }}$ is a constant of $298.15 \mathrm{~K}[5]$.

Fluent uses a different form of the energy equation when analyzing solid re-

gions of the model. For these solid regions, Fluent uses the following form of the energy equation,

$$
\frac{\partial}{\partial t}(\rho h)+\nabla \cdot(\vec{v}(\rho h))=\nabla \cdot(k \nabla T)+S_{h}
$$

where $k$ is the thermal conductivity of the solid material[5]. The first term on the right hand side of equation represents the heat flux due to conduction[5]. 


\subsubsection{P-1 Radiation}

Both in space and in the vacuum chamber, a substantial component of heat transfer on thrusters occurs as a result of radiation. The radiation of MiXI was modeled using Fluent's P-1 radiation model. Fluent provides five built-in radiation models to chose from and the P-1 model was chosen because of its reputation for providing good accuracy with the highest computational efficiency[1]. The P1 model is based on the spherical harmonics method of approximating radiative intensity $[20]$.

One of the downsides of the $\mathrm{P}-1$ model is that it tends to over predict radiative heat fluxes from localized sources[5]. Another downside of the P-1 model is that it's intended to be used for radiation in the presence of a participating media (ie. not in a vacuum), and tends to become less accurate when an optically thin media separates the hot and cold surfaces (which is the case inside the vacuum chamber) $[5,20]$.

Due to the obvious drawbacks of the P-1 model, the Surface-to-Surface model was also considered. The S2S model seemed like the obvious choice because it's intended to model radiative heat transfer within an enclosure without any participating media. However, the S2S model cannot be used in models that contain axissymmetric boundary conditions, which rules out it's use in this particular model[5]. Despite this limitation, an attempt was made to create a full model of the MiXI-vacuum chamber system without using symmetric boundary conditions. This attempt was made so that the $\mathrm{S} 2 \mathrm{~S}$ radiation model could be used for this project, but this attempt yielded very poor accuracy and ended up being more computationally expensive than the P-1 model. The poor accuracy is likely due to the complexity of the geometry and the relatively high number of surfaces 
radiating or absorbing heat. Additionally, the model that utilized S2S radiation proved to be very CPU-intensive because symmetric boundary conditions could not be applied, therefore nearly twice as many elements had to be analyzed. So despite all of its faults, the P-1 model was able to provide a better accuracy and computational efficiency than the S2S model.

When one of Fluent's radiation models is activated, the volumetric heat sources $\left(S_{h}\right)$ in eq. 4.4 and eq. 4.8 will include the radiation source term[5]. The equations used by the P-1 model to calculate these terms are discussed in the following sections.

\section{Radiative Transfer Equation}

Four of the five built-in radiation models available in Fluent (including the P-1 model) are designed to account for absorbing, emitting, and scattering media[5]. While this does not make up a huge component of radiation in this particular model, the effects are still analyzed as part of the P-1 radiation model and therefore, the relevant equations will be discussed. Fluent utilizes the radiative heat transfer equation (RTE) for absorbing, scattering, and emitting media at position $\vec{r}$ in the direction $\vec{s}$. This equation is defined as follows,

$$
\frac{d I(\vec{r}, \vec{s})}{d s}+\left(a+\sigma_{s}\right) I(\vec{r}, \vec{s})=a n^{2} \frac{\sigma T^{4}}{\pi}+\frac{\sigma_{s}}{4 \pi} \int_{0}^{4 \pi}\left(\vec{r}, \vec{s}^{\prime}\right) \Phi\left(\vec{s}^{\prime} \cdot \vec{s}\right) d \Omega^{\prime}
$$

where $\vec{r}$ is the position vector, $\vec{s}$ is the direction vector, $\vec{s}$ is the scattering direction vector, $s$ is the length of the path, $a$ is the absorption coefficient, $n$ is the refractive index, $\sigma_{s}$ is the scattering coefficient, $\sigma$ is the Boltzmann constant, $I$ is the radiation intensity, $T$ is the local temperature, $\Phi$ is the phase function, and $\Omega^{\prime}$ is the solid angle[5]. 


\section{Local Radiation Intensity}

The Ansys Fluent user manual describes the P-1 model the simplest version of the P-N model[5]. The P-1 model only uses four terms in the P-N series, the first one bing the local-radiation-intensity term which is defined by the following equation,

$$
\nabla \cdot(\Gamma \nabla G)-a G+4 a \sigma T^{4}=S_{G}
$$

where $a$ is the absorption coefficient, $\sigma$ is the scattering coefficient, $G$ is the incident radiation, $\sigma$ is the Stefan-Boltzmann constant, $T$ is the local temper-

ature, and $S_{G}$ is a radiation source[5]. The $\Gamma$ term is defined by the following equation,

$$
\Gamma=\frac{1}{\left(3\left(a+\sigma_{(s)}\right)-C \sigma_{s}\right)},
$$

where $C$ is the linear-anisotropic phase function coefficient and $\sigma_{s}$ is the scattering coefficient [5].

Equation 4.10 is the equation solved by Fluent to determine the local radiation intensity [5].

\section{Anisotropic Scattering}

In order to model anisotropic scattering, the P-1 model uses the following formula,

$$
\Phi\left(\vec{s}^{\prime} \cdot \vec{s}\right)=1+C \vec{s}^{\prime} \cdot \vec{s}
$$

Equation 4.12 is know as the linear-anisotropic scattering phase function[5]. 
In this equation, $\vec{s}$, is the unit vector in the direction of the scattering, $\vec{s}$ is a unit vector in the incident radiation direction, and $C$ is a coefficient that is a property of the fluid and can range in value from -1 to $1[5]$.

\section{P-1 Model at Walls}

The equation to compute heat flux for the energy equation and for the incident radiation equation boundary conditions is as follows,

$$
q_{r, w}=-\frac{\epsilon_{w}}{2\left(2-\sigma_{w}\right)}\left(4 \sigma T_{w}^{4}-G_{w}\right)
$$

In equation $4.13, \epsilon_{w}$ is the emissivity of the wall, $T_{w}$ is the local wall temperature, and $G_{w}$ is the incident radiation at the wall[5].

The fourth term of the P-1 model accounts for particulate effects that occur when the Fluent model includes a dispersed second phase of particles[5]. As this is not the case for the model created for this project, the equations for particulate effects will not be discussed.

\subsection{Geometry \& Mesh Generation}

The two-dimensional mesh was created from scratch using Ansys' ICEM mesh generation software. The geometry and mesh that was generated in ICEM is mostly rectangular - all of the lines make 90 degree angles with the lines they intersect. This means that a structured mesh can be used for this geometry, which

is desirable because structured meshes typically use memory more efficiently and lead to faster convergence[5]. The mesh that was used for this thermal model was created using ICEM's 'Surface Meshing' tool. This tool creates meshes that are technically unstructured, but certain mesh generation methods can be used 
to create de-facto structured meshes.

The final mesh took advantage of the fact that MiXI is axisymmetric. MiXI's symmetry about it's center axis allows us to assume that the temperature distribution is the same in all dimensions of the thruster. This means that only one half of one plane of the thruster needs to be modeled. Therefore, the final mesh only modeled the upper half of a two dimensional cut-away view of MiXI and the surrounding vacuum chamber. This mesh is shown in fig. 4.1.

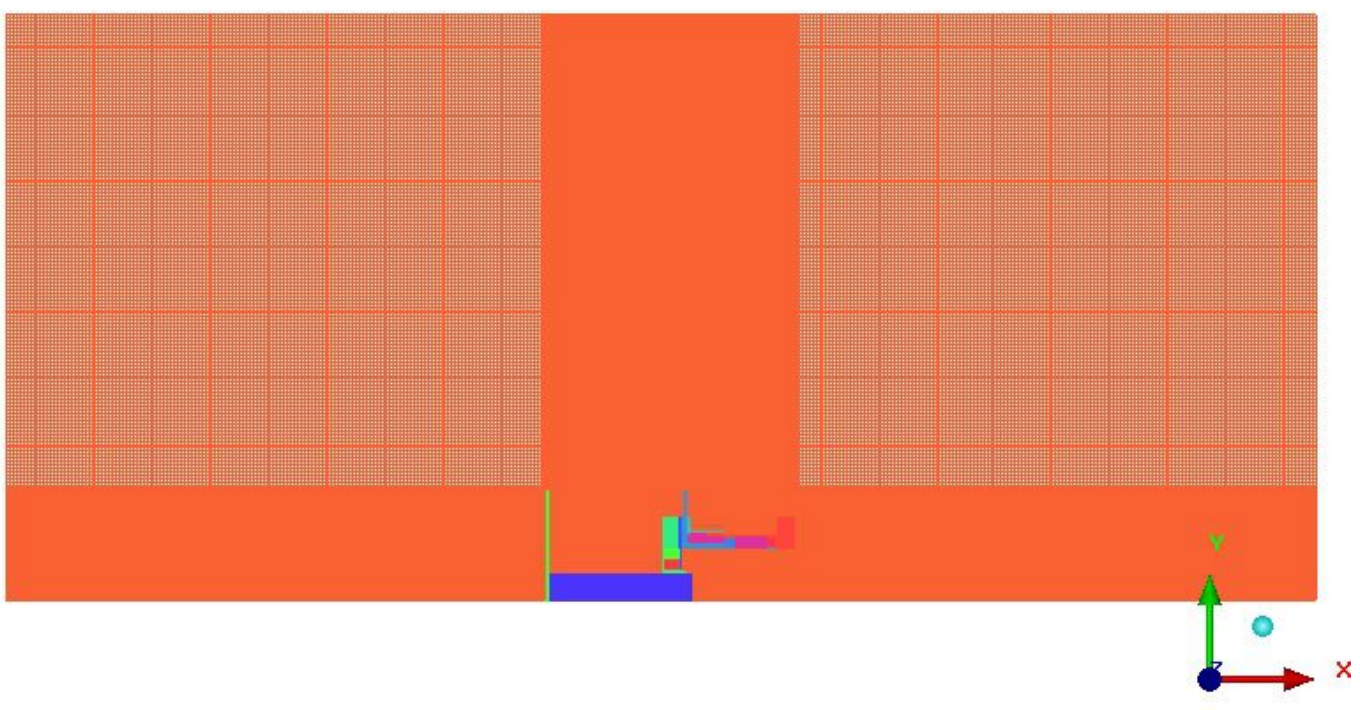

Figure 4.1: This image shows the mesh that was created in ICEM and read into Fluent. MiXI can be seen in the bottom-center of the image, the orange area surrounding MiXI is the interior of the vacuum chamber.

The mesh was generated using ICEM's Autoblock meshing function. The fineness of the mesh was determined by specifying a maximum height for each part of the mesh; the components that made up the hollow cathode were assigned 
a maximum cell height of 0.2 and the areas that were part of the vacuum environment were assigned a maximum cell height of 1 . This resulted in a structured mesh that was more fine in and around the solid components of the thruster and more course in the areas that are apart of the vacuum. The final mesh had a total of 289,080 cells, 292,842 nodes, and 581,977 faces.

As can be seen from fig. 4.2, the thermal model includes MiXI's anode, anode pole piece, anode cap piece, magnet shield, lower magnet mount, and grid isolation mount. Also included are the hollow cathode's electrode, the electrode isolation sheath, and the hollow cathode's chassis. Components of MiXI's setup that were not included are the screen and accelerator grids, and support equipment that was within the chamber, such as the electrical cables and plumbing for the propellant system.

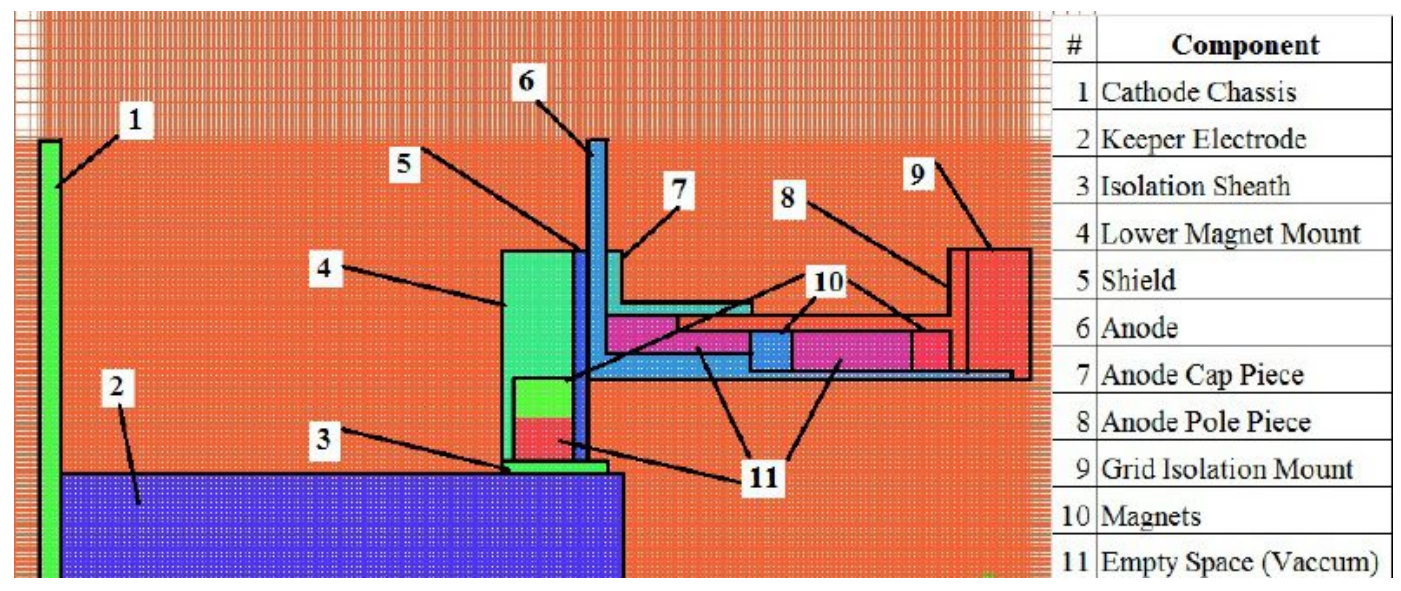

Figure 4.2: A close-up view of the mesh area containing MiXI with each part labeled.

The dimensions of the thruster and hollow cathode were taken from the twodimensional Pro/Engineer drawings created by Mr. Knapp when he designed MiXI-CP-V3. The dimensions of the thruster and cathode parts modeled in ICEM are stated in chapter 2 and are accurate to $0.1 \mathrm{~mm}$ of the actual parts 
dimensions. The dimensions of the chamber walls is less accurate because they had to be measured from the location of the thruster during testing. The distance between the walls of the vacuum chamber are $44.5 \mathrm{~cm} \pm 1 \mathrm{~cm}$ and the distance from the top of the vacuum chamber to the centerline of the thruster is $20.32 \mathrm{~cm}$ $\pm 1 \mathrm{~cm}$.

\subsubsection{Mesh Independence Study}

When numerical methods to analyze heat transfer or fluid flow within a discretized domain, it is important to ensure that the mesh is refined enough to produce an accurate solution, but not so refined that computational efficiency is sacrificed. To prove that this mesh is not too fine and not too course, a simple mesh independence study was conducted. In a grid independence study, the same problem is analyzed with multiple meshes of varying fineness.

For this study, a total of three meshes were analyzed. The first mesh is much courser than the mesh that was used in the final model, and the second mesh was much finer than the one that was used in the final model. The intermediate mesh is the mesh that was used for the final thermal model.

Table 4.1 shows the results of the grid independence study in addition to information on the size and computation time of the three meshes. The table shows that the difference of the solution between the fine and final meshes is only about $0-0.5{ }^{\circ} \mathrm{C}$. Additionally, the difference of the solutions of the intermediate mesh and the course mesh is much larger at about $0.5-3.1^{\circ} \mathrm{C}$. This shows that if the mesh were made to be more course, the accuracy of the solution may become compromised. However, refining the mesh any further will not yield better results while requiring more time to compute. This proves mesh independence. 
Table 4.1: Results of the mesh independence study. The minor difference between the results of the final mesh and the fine mesh proves grid independence of the final mesh.

\begin{tabular}{|c|c|c|c|}
\hline & Course & Fine & Intermediate \\
\hline \# of Cells & 150,195 & 387,902 & 289,080 \\
\hline Computation Time $(\mathrm{hrs})$ & 1.4 & 42 & 12.5 \\
\hline TC 1 Final $\left({ }^{\circ} \mathrm{C}\right)$ & 116.7 & 121.3 & 119.8 \\
\hline TC 2 Final $\left({ }^{\circ} \mathrm{C}\right)$ & 110.3 & 113.1 & 112.7 \\
\hline TC 3 Final $\left({ }^{\circ} \mathrm{C}\right)$ & 131.6 & 134.8 & 134.6 \\
\hline TC 4 Final $\left({ }^{\circ} \mathrm{C}\right)$ & 112.2 & 115.1 & 115.2 \\
\hline TC 5 Final $\left({ }^{\circ} \mathrm{C}\right)$ & 149.2 & 153.8 & 153.7 \\
\hline TC 6 Final $\left({ }^{\circ} \mathrm{C}\right)$ & 152.2 & 155.3 & 155.2 \\
\hline TC 7 Final $\left({ }^{\circ} \mathrm{C}\right)$ & 159.4 & 161.4 & 161.4 \\
\hline TC 8 Final $\left({ }^{\circ} \mathrm{C}\right)$ & 163.2 & 165.7 & 165.7 \\
\hline
\end{tabular}

Additionally, we can estimate the relative error of our thermal model from the results of our grid independence study. According to a paper by Dr. Ismail Celik of West Virginia University, approximate relative error can be determined with the equation,

$$
e_{\text {approx }}=\left|\frac{\phi_{\text {fine }}-\phi_{\text {int }}}{\phi_{\text {fine }}}\right|,
$$

where $e_{\text {approx }}$ is the approximate error, $\phi_{\text {fine }}$ is the final value of the metric of interest (temperature, in our case) from the refined mesh, $\phi_{i n t}$ is the final value of the metric of interest from the courser mesh[6]. Using eq. 4.14, we estimate that the temperature at any point in our model will have an error of about $1.34 \%$.

\subsection{Thermal Model of MiXI-CP-V3}

After the mesh was generated in ANSYS ICEM, it was read into ANSYS Fluent where it could be analyzed. This section describes how the thermal model was 
set up and analyzed after it was read into Fluent.

\subsubsection{Assumptions}

The thermal environment of an ion thruster can be very difficult to model. In order to simplify the modeling process, several assumptions were made. These assumptions will be discussed in this section.

\section{Phases of Operation}

As discussed in section 3.3, our test data shows that there are four phases of thruster operation: hollow cathode heating, ignition, nominal cathode operation, and cool down. For simplicity, the thermal model only considered the cathode heating phase and the nominal operation phase. The cool down phase was omitted because it's not important for the research being conducted. The ignition phase was omitted because the rate of temperature change for this phase was identical to the cathode heating phase.

\section{Time Rate of Temperature Change}

At the beginning of this project, it was assumed that the best way to model the thermal environment of the thruster was to model the plasma within the discharge chamber. However, this proved to be impossible to accomplish without Fluent's Magnetohydrodynamics (MHD) module. Ansys produces an add-on module for Fluent that has some ability to model plasma[5]. Unfortunately, Cal Poly does not own a license for this module and the cost of a single license far outweighed its potential usefulness. Attempts were then made to model the plasma as a simple fluid but this could not accurately model the plasma flow within the chamber 
because it ignored the effects of the magnetic field.

Conveniently, the test data showed very consistent time rates of temperature change. It was determined that the same objectives could be met by simply applying an empirically determined temperature change as a boundary condition. The hollow cathode was modeled as a solid surface and its empirically observed temperature was applied to the model as a fixed condition. Similarly, the nominal operations phase was modeled by applying a temperature at the walls of the discharge chamber in addition to a temperature at the cathode. This was possible for the transient case because the temperature of the thruster at various points was known at every moment of the thruster's operation. The temperature data acquired from the hollow cathode and thruster during testing was modeled as a polynomial function of time. This polynomial expression for temperature as a function of time was incorporated into the Fluent model via a user designed function (UDF) and set as a boundary condition to the electrode of the hollow cathode and the walls of the discharge chamber. This provided a solution that most closely resembled the empirically observed temperature data and therefore, the flow of plasma within the chamber was not modeled.

\subsubsection{User Defined Functions}

ANSYS Fluent has a feature that allows users to create custom functions in order to enhance Fluent's capabilities[5]. These User Defined Functions (UDF) must be written in the $\mathrm{C}$ programming language and are compiled in Fluent using Fluent's built-in compiler[5]. A total of four UDF's were used in this thermal model. One UDF was used to define the temperature boundary condition of the hollow cathode during cathode operations. One UDF defined the temperature boundary condition at the walls of the discharge chamber to simulate the heating 
due to plasma in the chamber. The last two UDF's were used to define the thermal conductivity of steel and Macor in order to account for the fact that material properties do not stay constant as temperature changes. All of these functions will be described in this subsection while the code for the UDF's can be seen in Appendix A.

\section{Hollow Cathode UDF}

To model the heating phase of the thruster's operation, a UDF was created and interpreted in Fluent. The UDF simulated the temperature change of the outer surface of the hollow cathode throughout the thruster's operation. This function consisted of a polynomial equation that defined heater temperature as a function of time.

During thruster testing, a thermocouple was placed on the hollow cathode's electrode, outside of the thruster. The polynomial was derived from this test data and the coefficients were generated using MATLAB's polyfit function. Fluent requires that all interpreted UDF's be in SI units, so a conversion factor of 273.15 was added to convert the temperature from ${ }^{\circ} \mathrm{C}$ to $K$. The $14^{\text {th }}$ order polynomial equation can be found in Appendix A.

\section{Heating from Plasma UDF}

As stated previously, modeling plasma within the discharge chamber proved to be beyond the scope of this project. In order to avoid modeling plasma, the thruster's nominal operations phase was modeled by applying a temperature boundary condition to the walls of the discharge chamber. The temperature at the walls was defined by a UDF that modeled temperature change with respect 
to time. Similar to the Hollow Cathode UDF, this UDF utilized data collected during one of the initial tests of MiXI. Unlike the Hollow Cathode UDF, this UDF was not applied throughout the entire operation of the thruster; this UDF was only applied during the nominal operations phase (between the 16.5 and 23.5 minute marks of the overall operation). During testing, it was observed that the temperature variation with time was linear during this phase; this can be seen in fig. 3.10 and fig. 3.11 in the previous chapter.

Using a UDF to simulate the temperature change during nominal operations proved to be a convenient way around modeling plasma. Restricting the boundary conditions to the known temperature at a given point in time simulates the power deposited by the plasma without actually modeling the plasma. The main downside of this technique is that we cannot use the model to determine when the thruster's temperature profile will reach steady state. Since we only have experimental temperature data for the six minutes of thruster operations, the model becomes unreliable if it's used to model anything beyond that amount of time.

\section{Thermal Conductivity UDF}

The thermal conditions of all materials do not remain constant with large fluctuations of temperature. Because conduction is the primary component of heat transfer in this model, it is important to ensure that the thermal conductivity of all materials is as accurate as possible. For this reason, it was decided to account for variation in thermal conductivity with time.

Sixth order polynomial equations for thermal conductivity as a function of temperature were created for steel and Macor, the two materials used in MiXI. 
Experimental data showing the variation in thermal conductivity with temperature for stainless steel 303 in fig. 4.4, and for Macor in fig. 4.3.

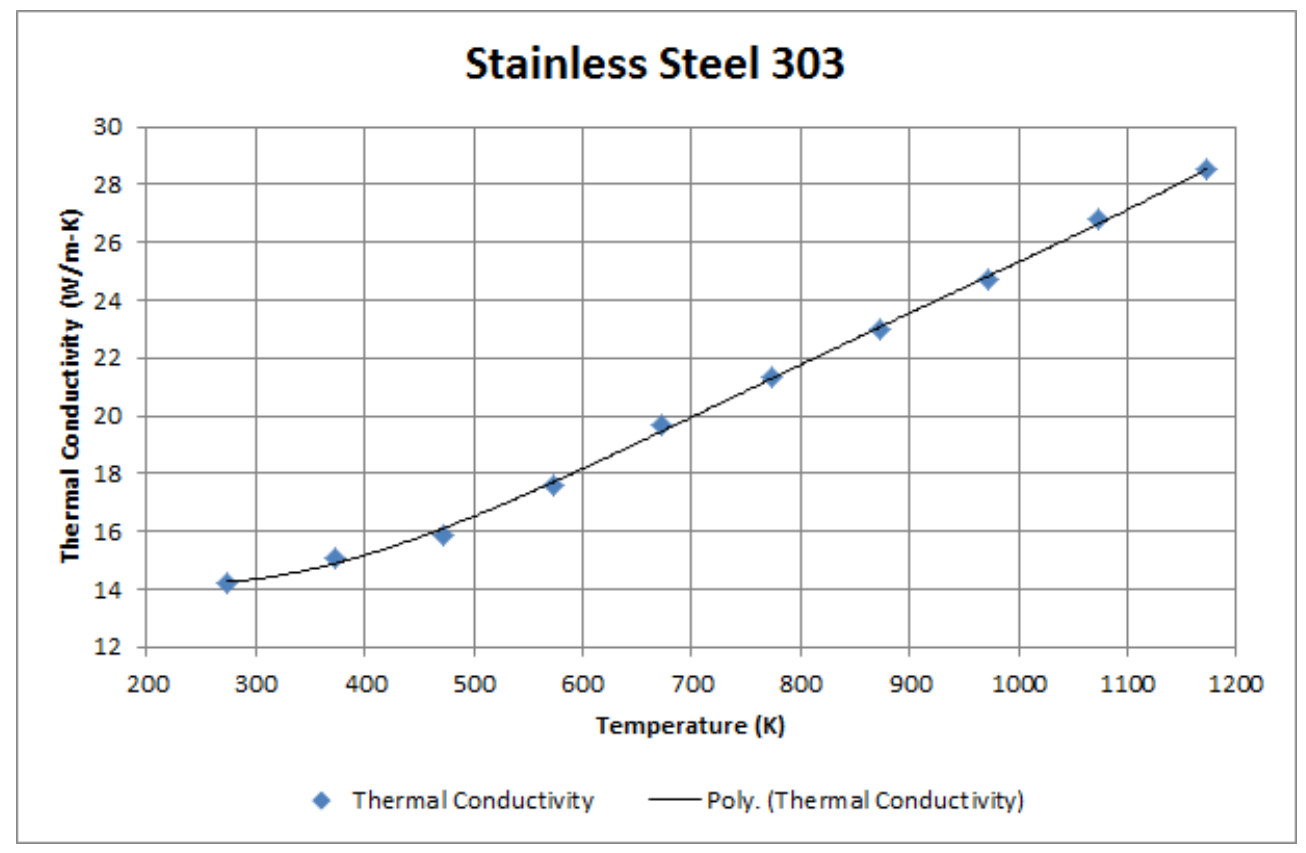

Figure 4.3: Thermal conductivity of stainless steel 303 as a function of temperature.[26] 


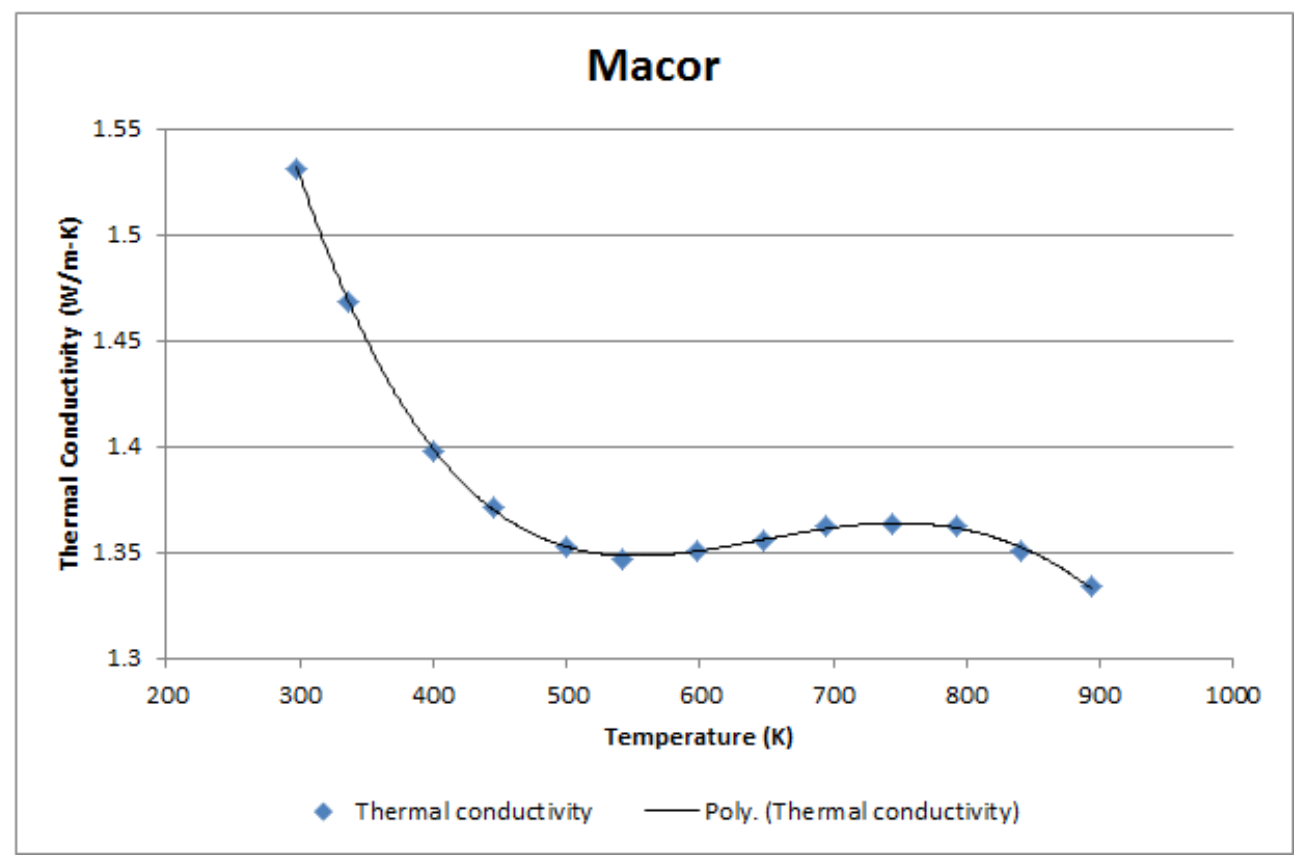

Figure 4.4: Thermal conductivity of Macor as a function of temperature.[3]

The $6^{\text {th }}$ order polynomial equations that define thermal conductivity for both Macor and Stainless Steel can be found in Appendix A.

\subsubsection{Thermal Contact Resistance}

Much of the heat propagating through MiXI is shunted at the interfaces of each component. This is due to the thermal contact resistance between each component. Contact resistance occurs because the surface of any material can never be perfectly flat; the surface of every solid has (sometimes microscopic) imperfections that leave the appearance of peaks and valleys. When two solid objects are pressed together, much of the surfaces will be in contact but tiny cavities will also exist between them. As a result of this imperfect contact, heat transferred between the two objects occurs primarily via conduction at the points of contact, with a smaller component coming from convection in the fluid (usually 
air) between the surfaces[13, 18, 12, 8]. The space between the surfaces where there is no contact is commonly known as the void space[12]. The phenomenon of contact resistance is illustrated in fig. 4.5.

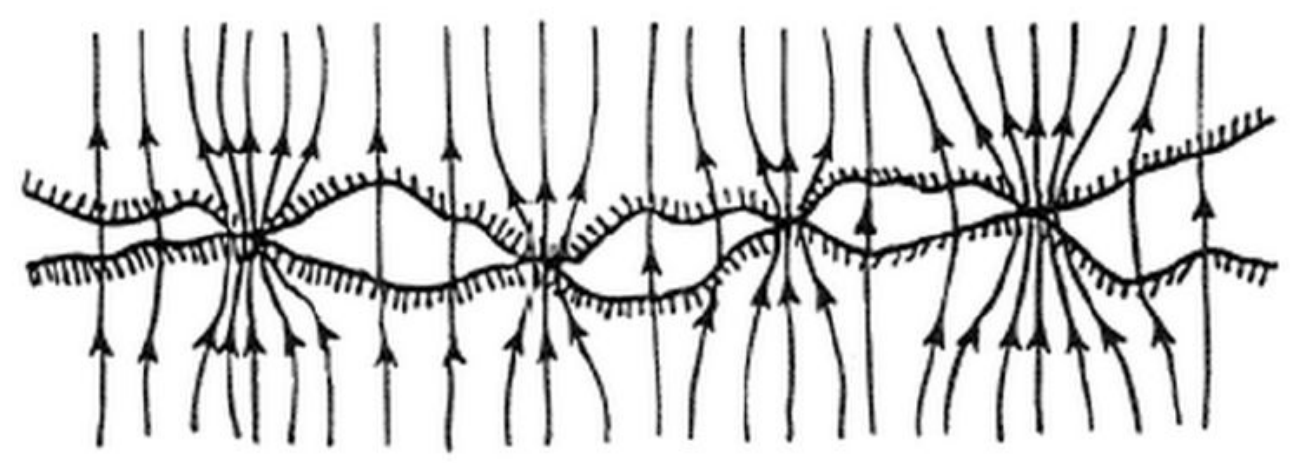

Figure 4.5: A close-up view of two solid surfaces in contact. The arrows illustrate how heat is transfered between the surfaces. The gaps between the surfaces is called the void space.[2]

The true thermal contact resistance is a notoriously difficult property to determine. Resistance varies significantly depending on the contact pressure, the surface roughness, the shape of the objects, and the material properties of the objects in contact. There is no reliable method for predicting contact resistance analytically, therefore it must be measured experimentally $[2,18,12,13]$. However, experimental studies have not yielded consistent results because some variables, like surface roughness, vary considerably with each specimen[2, 18, 12, 13].

In his book, Heat Transfer, Holman provides a simplified equation for estimating thermal contact conductance (the reciprocal of contact resistance)[12]. The equation for the coefficient of thermal contact conductance,

$$
h_{c}=\frac{1}{L_{g}}\left(\frac{A_{c}}{A} \frac{2 k_{A} k_{B}}{k_{A}+k_{B}}+\frac{A_{v}}{A} k_{f}\right),
$$

takes into account the total contact area and the distance between the two 
surfaces[12]. In eq. $4.15, L_{g}$ is the thickness of the void space, $A_{c}$ is the surface area that is in contact, $A_{v}$ is the area of the void space, $A$ is the total surface area, $k_{f}$ is the conductivity of the fluid in the void space, $k_{b}$ and $k_{b}$ are the conductivities of the surfaces that are in contact[12].

Equation 4.15 was used to approximate the contact resistance between the components of MiXI. Because the actual contact area can never be known for certain, a range of values was used for this variable. This resulted in a range of possible contact resistances between the surfaces that bracketed the values that were eventually used in the thermal model. The values for contact resistance that were eventually used were determined through an iterative process of guessing possible values, plugging them into the Fluent model, and comparing the result to the test data. After dozens of iterations, approximate values of the contact resistance were chosen. Figure 4.6 shows the interfaces where thermal contact resistance had to be calculated.

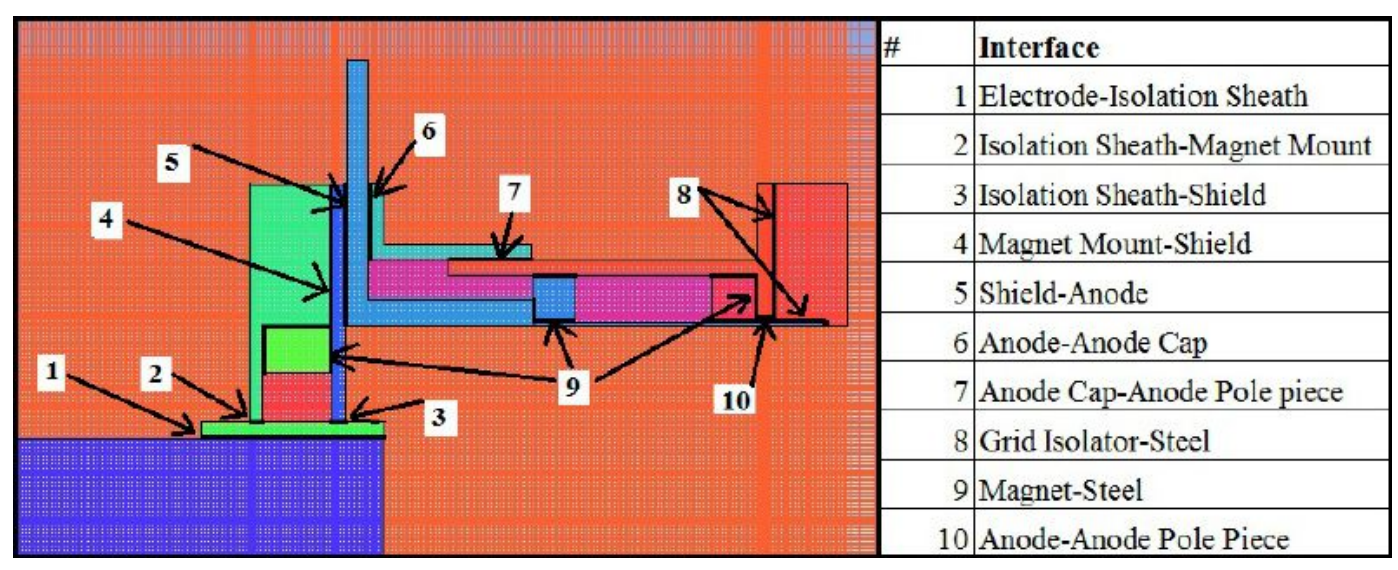

Figure 4.6: A close-up view of the mesh area containing MiXI with each part labeled. 
Table 4.2: Contact conductance between surfaces of MiXI parts.

\begin{tabular}{|c|c|c|}
\hline Interface & Analytical $h_{c}\left(\frac{m^{2}-K}{W}\right)$ & Final $R_{c}\left(\frac{m^{2}-K}{W}\right)$ \\
\hline Electrode-Sheath & $582.2-58.2 e 5$ & 1500 \\
\hline Sheath-Magnet Mount & $5.3-5.3 e 5$ & 10 \\
\hline Sheath-Shield & $5.3-5.3 e 5$ & 600 \\
\hline Magnet Mount-Shield & $59.1-3.5 e 5$ & 600 \\
\hline Shield-Anode & $59.1-3.5 e 5$ & 600 \\
\hline Anode-Cap & $59.1-3.5 e 5$ & 600 \\
\hline Anode-Pole Piece & $59.1-3.5 e 5$ & 200 \\
\hline Cap-Pole Piece & $59.1-3.5 e 5$ & 200 \\
\hline Pole Piece-Grid Isolator & $5.3-5.3 e 5$ & 600 \\
\hline
\end{tabular}

The values of the calculated contact conductance and the final values that were used in the model, are shown in table 4.2. Calculating contact conductance from eq. 4.15 provided a good starting point for the initial guesses and also verified that the guesses were reasonable. The large range in analytical conductances is due to the large uncertainty in $A_{c}$ and $L_{g}$; when presenting eq. 4.15 , Holman states, "The main problem with this simple theory is that it is extremely difficult to determine effective values of $A_{c}, A_{v}$, and $L_{g}$ for surfaces in contact."[12]. For our calculations, values of $L_{g}$ were taken from a range of surface roughness measurements found in [12] and [18]; the value for $L_{g}$ ranged from $0.5 \mu m$ to $3.0 \mu m$ for the steel interfaces and $0.5 \mu m$ to $3.0 \mu m$ for the Macor interfaces. The values of $A_{c}$ ranged from a fraction of the total area $(A * 1 e-3)$ to the entire area $(\mathrm{A})$. The conductivity of the void space was assumed to be zero since MiXI was in a vacuum.

Thermal contact resistance can be modeled in Fluent by specifying unique material for that interface. The thermal resistance can be modeled using the 
equation,

$$
R_{c}=\frac{\Delta x}{k}=\frac{1}{h_{c}},
$$

where $k$ is thermal conductivity and $\Delta x$ is the wall thickness[5]. When setting the boundary conditions in Fluent, one simply applies the wall thickness and thermal conductivity that equates to eq. 4.16. For example, if the contact resistance is $5 e^{-4} \frac{m^{2} K}{W}$ one may set the wall thickness to one meter and the thermal conductivity to $2000 \frac{W}{m-K}$. The other properties of this interface, such as density and heat capacity, are negligible.

\subsubsection{Material Properties}

One of the first steps of setting up a model in Fluent is to specify the properties of the materials being used. The properties of the materials used to model the MiXI-vacuum chamber system are shown in table 4.3. It should be noted that steel was used to make most of MiXI's parts, however, not the same type of steel was used for each part. The anode, the anode cap piece, and the shield were all made from stainless steel 303; because the specific grade of steel used to make these parts is known, we can look up their thermal properties. The steel used to make the anode pole piece, the magnet mount, and the cathode chassis is simply referred to as 'ferrous steel' by previous users of MiXI. Therefore, the model assumed that all the steel components of MiXI were made from stainless steel 303. 
Table 4.3: Thermal Properties of Materials[26, 12, 8, 13].

\begin{tabular}{|c|c|c|c|}
\hline Material & $\rho\left(\frac{\mathrm{kg}}{\mathrm{m}^{3}}\right)$ & $C_{P}$ & $k\left(\frac{\mathrm{W}}{\mathrm{m}-\mathrm{K}}\right)$ \\
\hline Stainless Steel 303 & 8027 & 500 & Temperature Dependant \\
\hline Macor & 2520 & 790 & Temperature Dependant \\
\hline Pyrex & 2230 & 750 & 1.1 \\
\hline Samarium-Cobalt 17 & 8400 & 335 & 12 \\
\hline Graphite & 1950 & 830 & 470 \\
\hline
\end{tabular}

As stated in section 4.3.2, the thermal conductivity of Macor and steel varies with temperature. Rather than modeling this as a constant, a UDF was used to model how thermal conductivity changes with temperature.

As stated in section 4.3.3, thermal contact resistance is modeled by designating a specific material for the coupled walls at the interfaces. When designating a contact resistance in Fluent, the density and heat capacity of the wall's material is ultimately irrelevant as long as it's greater than zero. For this thermal model, the density and heat capacity was set to one, and the wall thickness was set to $1 \mathrm{~m}$. The material properties of these walls are described in table 4.4.

Table 4.4: Thermal Properties of Part Interfaces.

\begin{tabular}{|c|c|c|c|c|}
\hline Interface & $C_{P}$ & $\rho\left(\frac{\mathrm{kg}}{\mathrm{m}^{3}}\right)$ & $k\left(\frac{\mathrm{W}}{\mathrm{m}-K}\right)$ & $\Delta x(\mathrm{~m})$ \\
\hline Electrode-Sheath & 1 & 1 & 1500 & 1 \\
\hline Sheath-Magnet Mount & 1 & 1 & 10 & 1 \\
\hline Sheath-Shield & 1 & 1 & 10 & 1 \\
\hline Magnet Mount-Shield & 1 & 1 & 600 & 1 \\
\hline Shield-Anode & 1 & 1 & 600 & 1 \\
\hline Anode-Cap & 1 & 1 & 600 & 1 \\
\hline Anode-Pole Piece & 1 & 1 & 200 & 1 \\
\hline Cap-Pole Piece & 1 & 1 & 200 & 1 \\
\hline Pole Piece-Grid Isolator & 1 & 1 & 600 & 1 \\
\hline
\end{tabular}




\section{Boundary Conditions \& Initial Conditions}

Once the thermal properties of the materials has been specified, the next step in setting up a Fluent model is to assign boundary conditions and cell zone conditions. Cell zone conditions are assigned to surfaces and volumes (for example, the anode or the interior of the vacuum chamber), while boundary conditions apply to the walls of the parts (such as the walls of the vacuum chamber or the interfaces between parts).

Table 4.5: Cell Zone and Boundary Conditions.

\begin{tabular}{|c|c|c|}
\hline Part & Boundary Condition & Material \\
\hline Vacuum & Fluid & Air \\
\hline Cathode Chassis & Solid & Steel \\
\hline Keeper Electrode & Solid & Steel \\
\hline Isolation Sheath & Solid & Macor \\
\hline Magnet Mount & Solid & Steel \\
\hline Shield & Solid & Steel \\
\hline Anode & Solid & Steel \\
\hline Cap & Solid & Steel \\
\hline Pole Piece & Solid & Steel \\
\hline Grid Isolator & Solid & Macor \\
\hline Magnet & Solid & SmCo17 \\
\hline Chamber Walls & Coupled Wall & Pyrex \\
\hline Chamber Top & Coupled Wall & Steel \\
\hline MiXI Centerline & Axis & - \\
\hline Exposed Surfaces & Coupled Wall & - \\
\hline Part Interfaces & Coupled Wall & - \\
\hline
\end{tabular}

Table 4.5 shows the cell zone and boundary conditions of the parts that were modeled. For the sake of brevity, the boundary conditions of each individual wall in the model is not shown in the table. The surfaces of the parts that are exposed to the vacuum (such as the exterior of the Pole Piece or the keeper electrode) are fall into the "Exposed Surfaces" category in the table and are modeled as 
coupled walls. The interfaces between each part (such as the interface between the anode and the shield) are listed as "Part Interfaces" in the table and are also modeled as coupled walls.

Table 4.6: Emissivities of Exposed Surfaces[20, 13, 12].

\begin{tabular}{|c|c|c|c|}
\hline Surface & Material & Theoretical Emiss & Actual Emiss \\
\hline Chassis & Steel & $0.74-0.87$ & 0.8 \\
\hline Electrode & Graphite & 0.8 & 0.8 \\
\hline Isolation Sheath & Macor & 0.9 & 0.9 \\
\hline Magnet Mount & Steel & $0.74-0.87$ & 0.8 \\
\hline Shield & Steel & $0.74-0.87$ & 0.8 \\
\hline Anode & Steel & $0.74-0.87$ & 0.74 \\
\hline Pole Piece & Steel & $0.74-0.87$ & 0.74 \\
\hline Cap Piece & Steel & $0.74-0.87$ & 0.8 \\
\hline Grid Isolator & Macor & 0.9 & 0.9 \\
\hline Chamber Top & Steel & $0.54-0.87$ & 0.6 \\
\hline Chamber Walls & Pyrex & $0.85-0.95$ & 0.9 \\
\hline
\end{tabular}

Table 4.6 shows the properties of the walls that are exposed to the vacuum environment. Like contact resistance, the values of emissivity can vary from specimen to specimen because of the differences in surface roughness[13]. The range of 'Theoretical Emissivities' listed in table 4.6 were pulled from tables in various textbooks while values for the 'Actual Emissivity' were determined by iteratively varying the value of the emissivity in the Fluent model. The values listed in the table for 'Actual Emissivity' provided a solution closest to the experimental results.

In order to model the vacuum environment in Fluent the pressure of the fluid environment was set to 1e-6 Torr, which roughly corresponds to the gauge pressure within the chamber during testing. The initial temperature for all of the parts was set to $31.5{ }^{\circ} \mathrm{C}$, which was the average temperature of the initial 
thermocouple data that was collected during testing.

\subsubsection{Solution Methods, Initialization, \& Calculation}

Eight monitors were set up to record the temperature at the locations of the thermocouples and write the temperatures data to a .txt file. The locations of the monitors is shown in fig. 4.7.

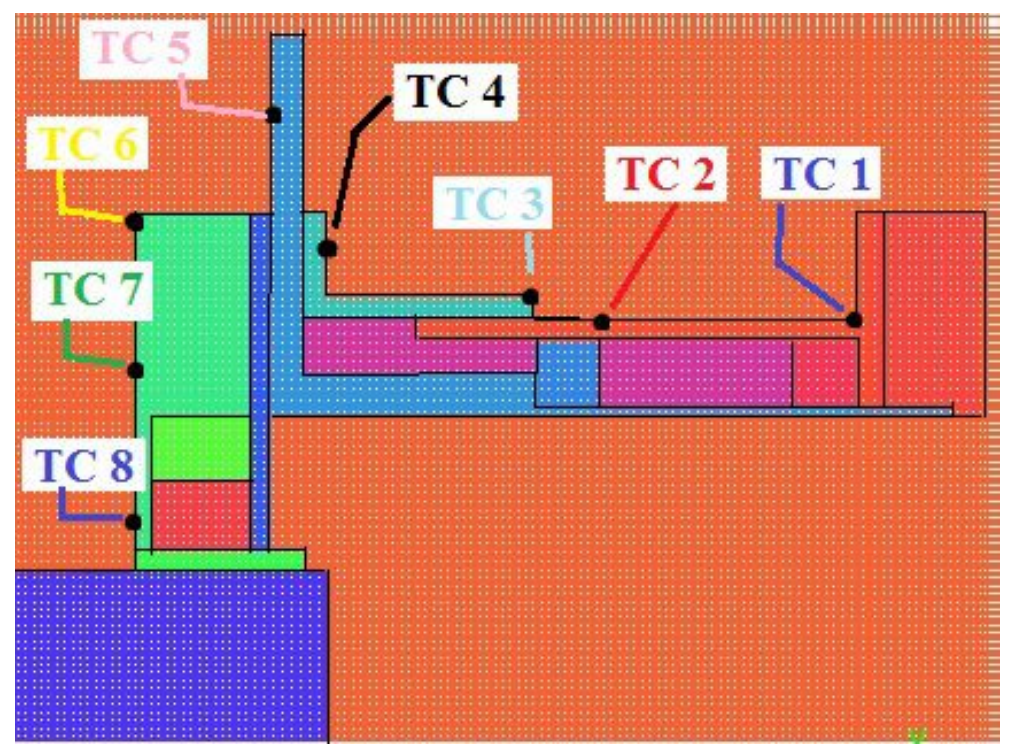

Figure 4.7: A cutaway view of a hollow cathode without the keeper electrode.

After the BC's, IC's, material thermal properties, and solution methods were specified, the model could be analyzed in Fluent. This analysis utilized a pressure based solver in transient mode, so the once the problem was initialized, the specific number of time steps had to be specified. In order for the theoretical data to accurately depict our test data, the Fluent model had to follow the same timeline as our experimental procedure. Before operating MiXI in the chamber, the hollow cathode's heater was turned on for 15 minutes before the process of igniting the cathode began. The ignition process consistently took about 1.5 
minutes to complete, at which point the cathode enter a self-sustaining mode and the heater could be shut down. To simulate all this, the first phase of the Fluent model was set for 16.5 minutes or 990 timesteps (one second per timestep). The monitor data for this phase of the thermal model is shown in fig. 4.8.

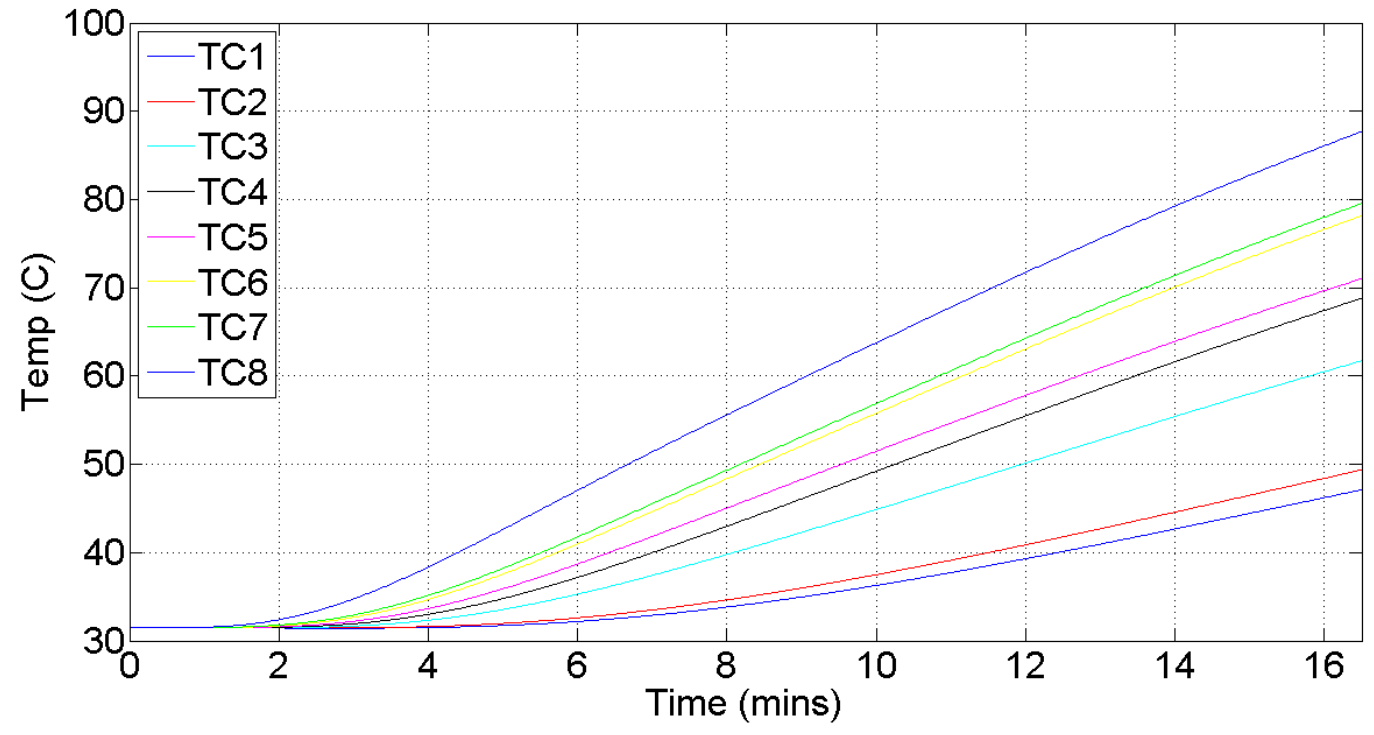

Figure 4.8: This image shows the temperature at each thermocouple location plotted against time for the heating phase of thruster operation.

When Fluent had completed the calculations for the heating phase, the boundary conditions at the inner wall of the anode and the inner wall of the shield were changed; fig. 4.9 indicates where the boundary conditions were changed. 


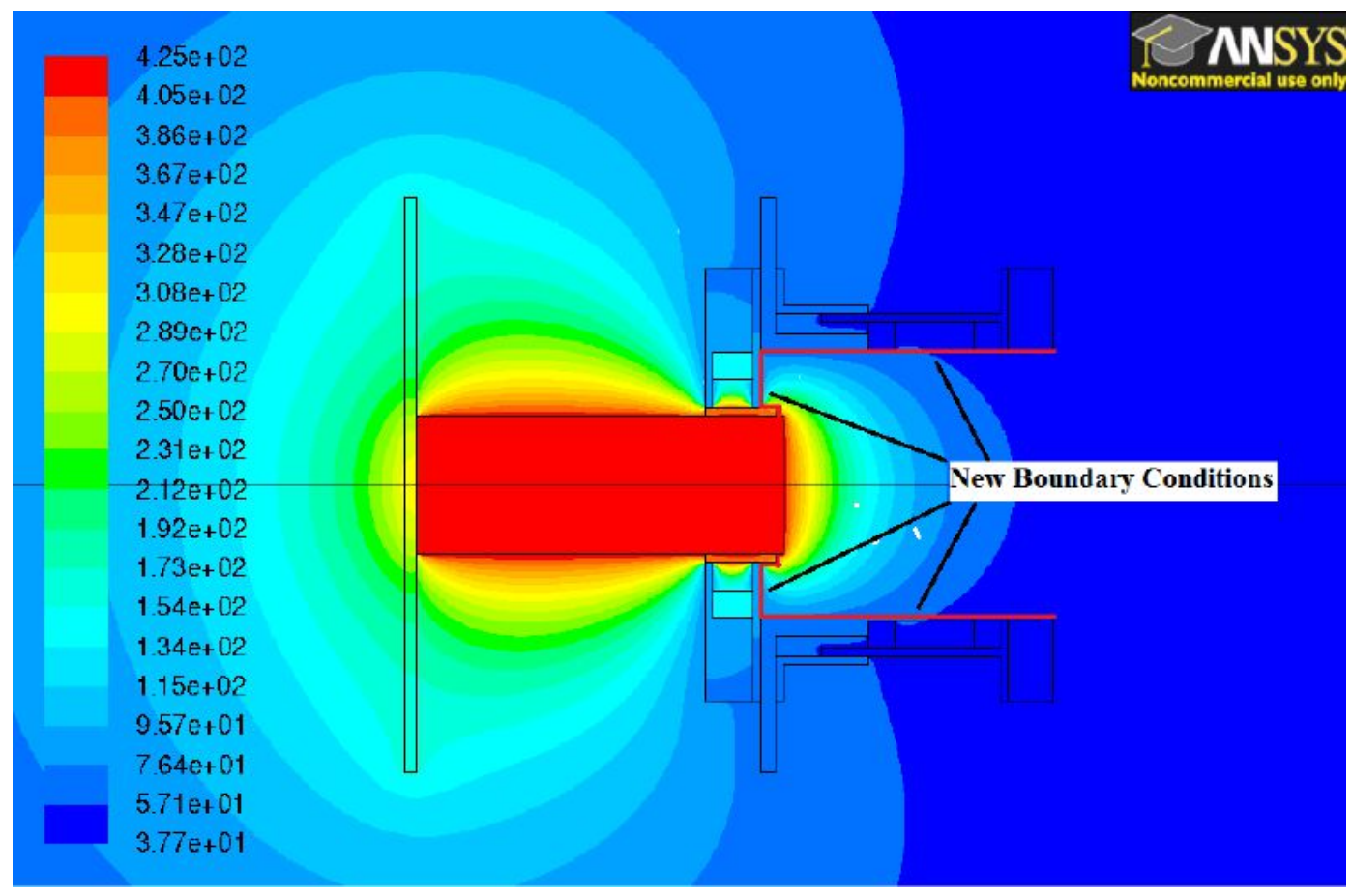

Figure 4.9: A contour plot of MiXI's temperature profile after the heating phase, indicating what boundary conditions were changed at that time.

At both walls the thermal boundary condition was changed to a temperature boundary condition. As mentioned in section 4.3.2, the temperature change at the wall was set as the time rate of change of the temperature that was observed during testing. This was accomplished by using a UDF to specify the wall temperature, rather than a constant temperature or a heat flux. Once the new boundary conditions had been specified, the number of timesteps was reset to 360 (6 minutes) and the calculation was resumed.

\subsection{Results \& Validation}

This section presents the results of the case described in the previous section and compares them to the experimental data that is described in chapter 3. Contour 
plots of temperature were created at several points of the thruster's operation. Figure 4.10 shows MiXI's thermal environment at the time of cathode ignition, 16.5 minutes after the heater was turned on.

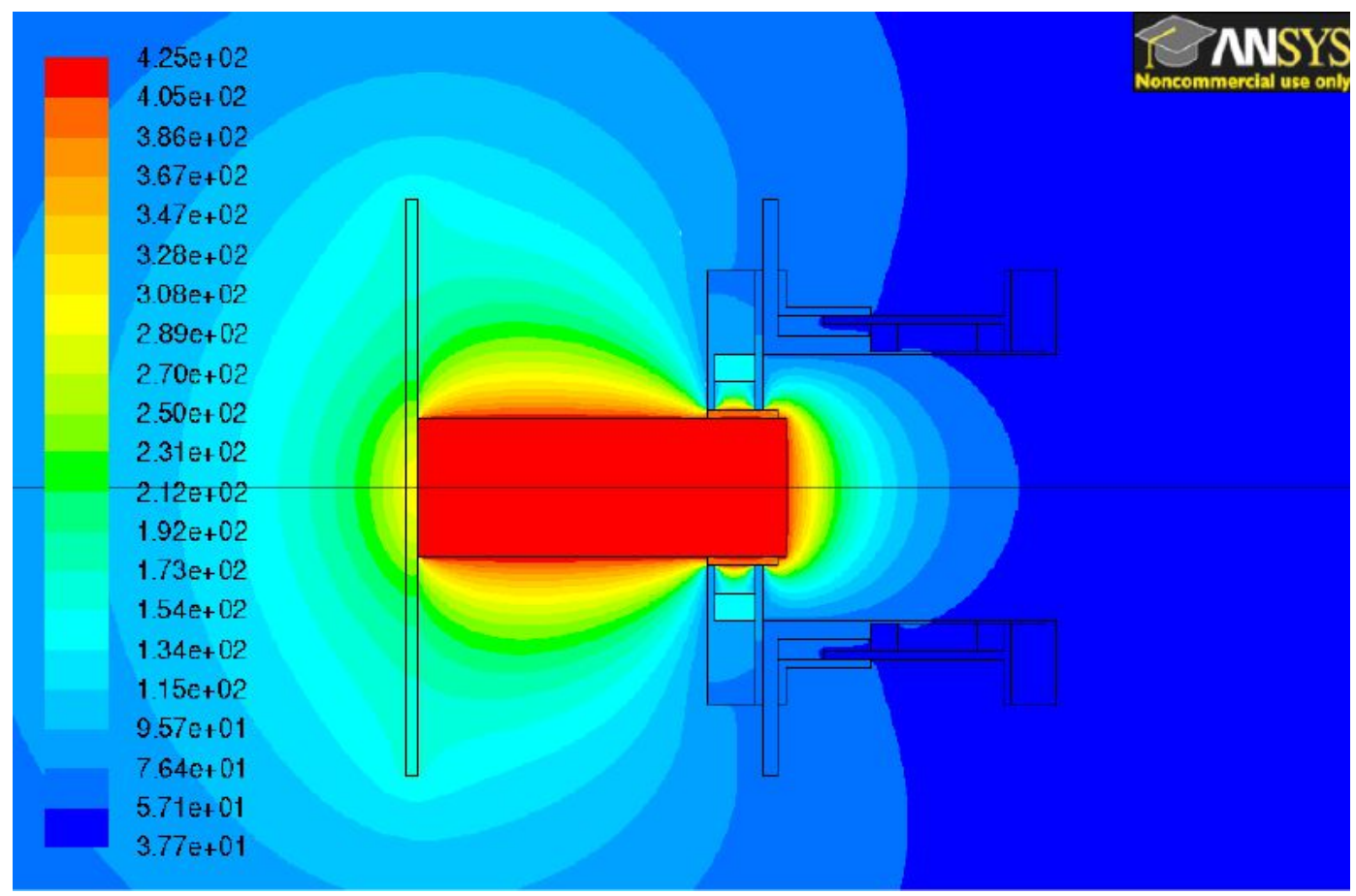

Figure 4.10: Contour plot illustrating the temperature profile of MiXI after the heating phase of operations.

Figure 4.11 shows MiXI's thermal environment after 6 minutes of cathode operation, 22.5 minutes after the heater was turned on. 


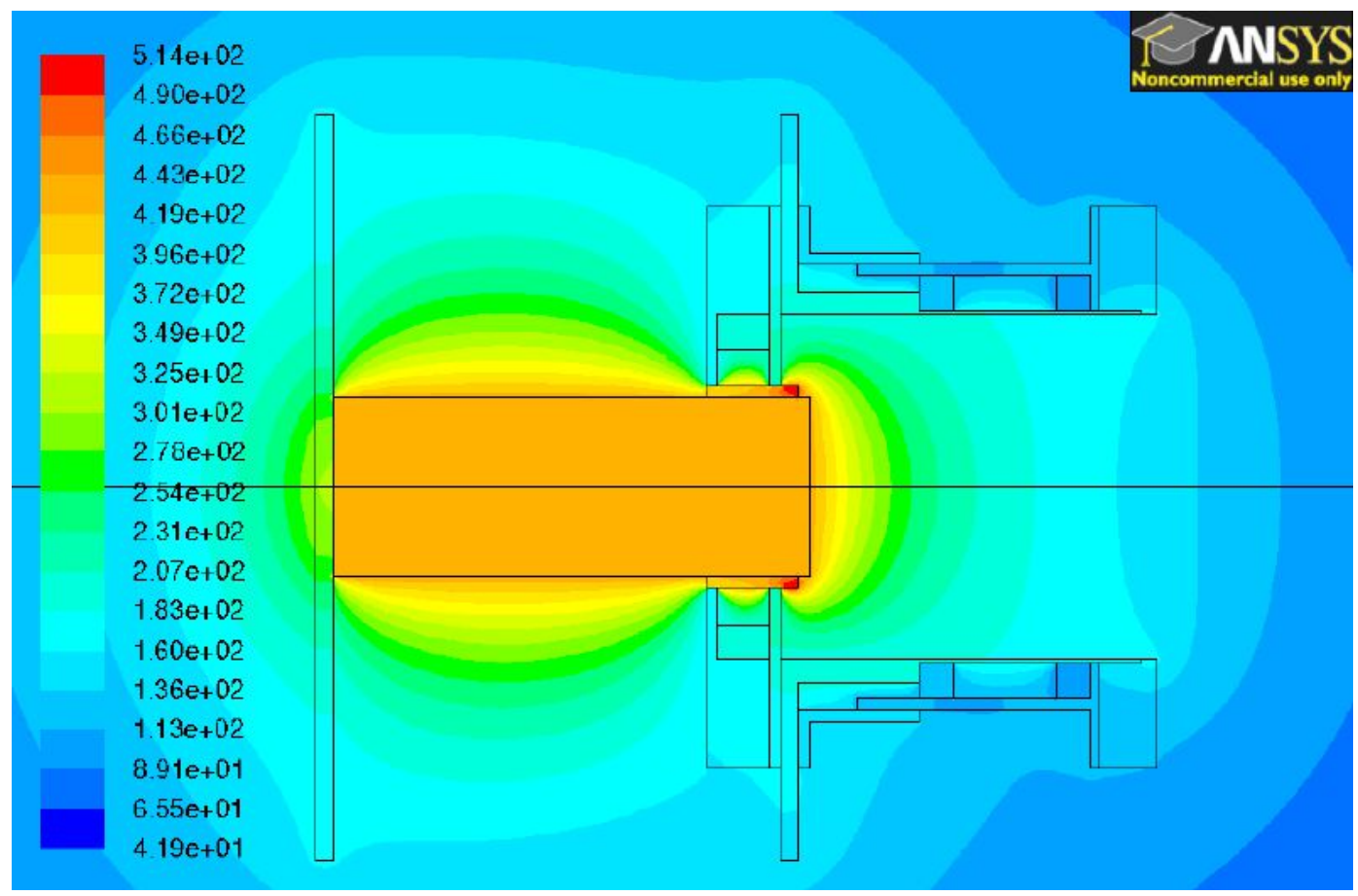

Figure 4.11: Contour plot illustrating the temperature profile of MiXI at time of cathode shutdown.

Figure 4.12 shows the temperature at the various thermocouple locations plotted against time. Both fig. 4.11 and fig. 4.12 indicates that the model predicts significantly lower temperatures at thermocouples 6,7 , and 8 . This is due to Fluent's inaccuracy in predicting surface heating to radiative heat transfer. The temperature data that was collected during testing indicates that thermocouples 6,7 , and particularly 8 , heat up much faster than the other five thermocouples. In chapter 3 , it was hypothesized that this is due to the heat that is being radiated from the surface of the electrode. This conclusion was determined because the distance between thermocouples 6,7 , and 8 is only $7 \mathrm{~mm}$, and the temperature difference is about $70^{\circ} \mathrm{C}$ at the time the cathode is ignited. This temperature gradient far too large to be only the result of conduction heat transfer. The Fluent model indicates that the temperature at these thermocouples is less than $90{ }^{\circ} \mathrm{C}$ at that time; far less then our experimental data. It should also be noted, 
that Fluent indicates that the temperature just a few millimeters off the surface of thermocouple 8 is about $230{ }^{\circ} \mathrm{C}$, approximately the same temperature indicated by thermocouple 8 at the time of cathode ignition.

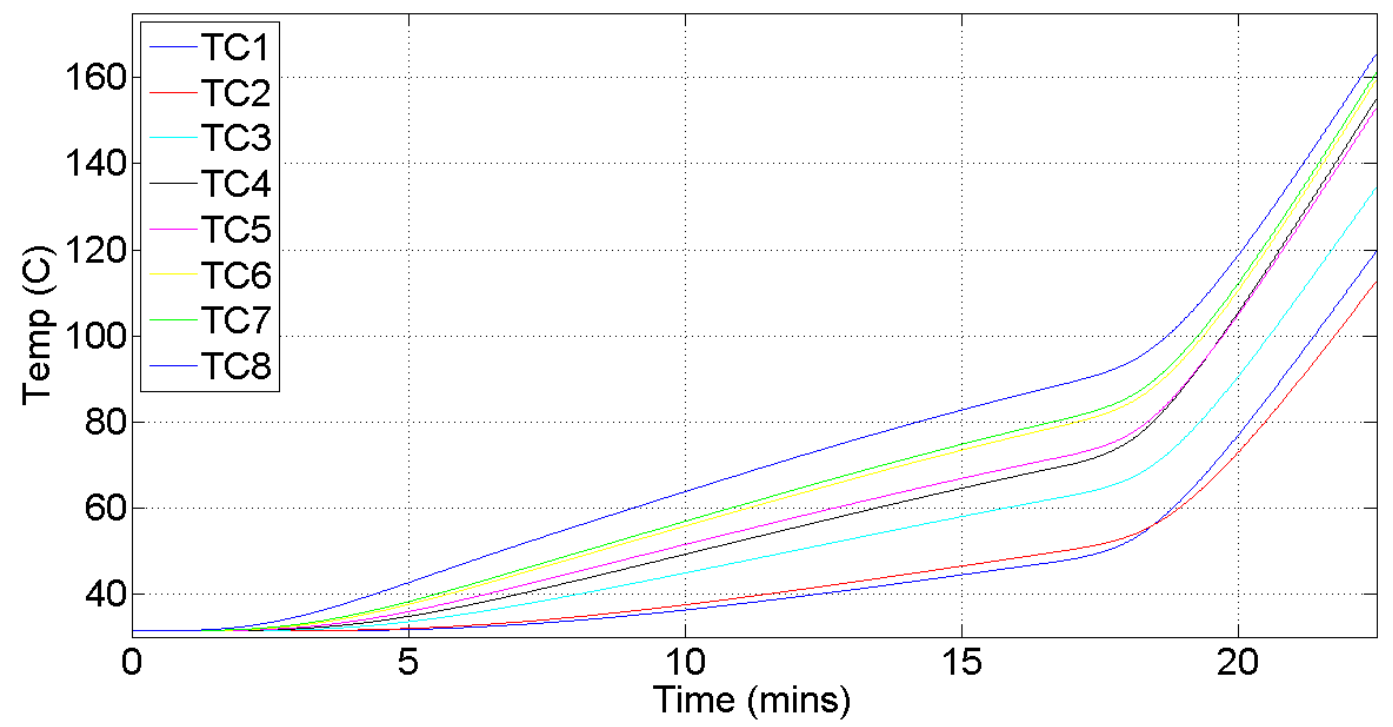

Figure 4.12: This image shows the temperature at each thermocouple location plotted against time.

Because the thermal model does not provide an reliable enough assessment of radiative heat transfer, it was decided to toss out the data for thermocouples 6,7 , and 8. Figure 4.13 shows temperature data at thermocouples 1-5 superimposed over the experimental temperature data that was recorded during testing. The thin lines indicate data that was recorded during testing and the thicker lines indicate data from the thermal model. The figure shows that the thermal model follows the same trends as the empirical data throughout MiXI's operating time. You will notice, however, that the data from TC5 starts to diverge at about 12 minutes into the heating phase. We can see that, at the time of cathode ignition, the thermal model predicts the temperature at TC5 to be $10^{\circ} \mathrm{C}$ less than the actual empirical data. This is due to the P-1 model's under-prediction of radiative heat transfer at surfaces; the same phenomenon that rendered the 
data from TC's 6,7 , and 8 inaccurate.

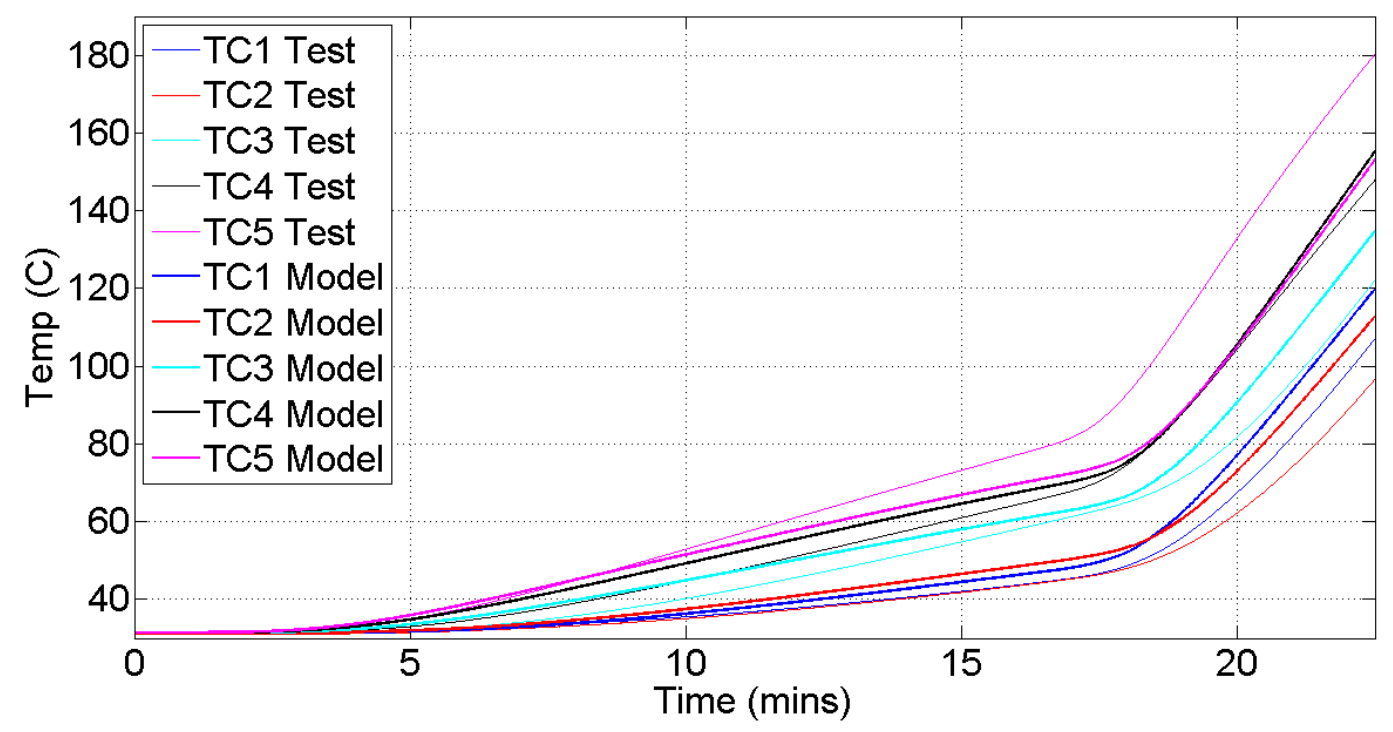

Figure 4.13: This image shows the temperature at each thermocouple location plotted against time.

Table 4.7 shows compares the theoretical temperatures to the actual temperatures at different stages of the operation. The theoretical temperatures have an error of about $1.34 \%$ according to the mesh independence study. With the exception of thermocouple 5 the thermal model predicts thermocouple temperatures within about $12{ }^{\circ} \mathrm{C}$ of temperatures that were measured during testing. This is close to our goal of $12{ }^{\circ} \mathrm{C}$ at these locations, the standard set by Mr. Van Noord's model of the NEXT thruster[21].

Table 4.7: Theoretical Temperatures vs Experimental Temperatures. Theoretical temperatures have an approximate error of $1.34 \%$.

\begin{tabular}{|c|c|c|c|c|c|c|c|c|}
\hline Time (min) & \multicolumn{2}{|c|}{10} & \multicolumn{2}{c|}{16.5} & \multicolumn{2}{c|}{20} & \multicolumn{2}{c|}{22.5} \\
\hline & Theo & Exp & Theo & Exp & Theo & Exp & Theo & Exp \\
\hline TC 1 $\left({ }^{\circ} \mathrm{C}\right)$ & 36.3 & 35.5 & 47.1 & 44.5 & 77.0 & 67.3 & 119.8 & 107.0 \\
\hline TC 2 $\left({ }^{\circ} \mathrm{C}\right)$ & 37.5 & 35.06 & 49.3 & 44.4 & 72.9 & 62.1 & 112.7 & 96.6 \\
\hline TC 3 $\left({ }^{\circ} \mathrm{C}\right)$ & 44.9 & 40.2 & 61.7 & 59.6 & 90.6 & 81.6 & 134.6 & 122.0 \\
\hline TC 4 $\left({ }^{\circ} \mathrm{C}\right)$ & 49.2 & 44.7 & 68.8 & 66.0 & 105.5 & 104.2 & 115.2 & 147.8 \\
\hline TC 5 $\left({ }^{\circ} \mathrm{C}\right)$ & 51.5 & 52.9 & 71.0 & 79.1 & 105.0 & 132.9 & 153.7 & 180.2 \\
\hline
\end{tabular}


The differences between the theoretical and experimental data can be attributed to many different assumptions made during the modeling process. One of the biggest contributors to the discrepancy is likely due to the failure of the P-1 radiation model to accurately predict the radiation temperatures at the thruster's surface. In his book Radiative Heat Transfer, Modest states, "... The P-1 approximation may be substantially in error in optically thin media with strongly anisotropic intensity distributions, in particular in multidimensional geometries with large aspect rations...and/or when surface emission dominates over medium emission." [20]. Modest then uses an example problem to point out that the results of the P-1 method are often least accurate in areas closest to walls[20]. The theoretical data for thermocouples 6,7 , and 8 was not compared to the corresponding experimental data because of this issue with the $\mathrm{P}-1$ radiation model.

Additionally, our two-dimensional model ignores many of the intricacies of the interior of the vacuum chamber. The $2 \mathrm{D}$ model assumes that the vacuum chamber is rectangular and its walls are opaque. In reality, the chamber is cylindrical and the walls are clear, which adds to the inaccuracy of the radiation prediction. Other sources of error include the uncertainty of the thermal contact resistances at the interfaces, and the uncertainties of the emissivities at the surfaces. The actual resistance and emissivity can never be known for sure, and the values used in the model are estimates that were refined through guess-and-check.

The model does not always predict the precise temperatures at the thermocouple locations, but the transient temperature changes as indicated by the model follows the same trend to the empirical data. Therefore, the model will be useful in determining whether or not certain methods of postponing magnetic degaussing will be feasible. 


\section{Postponement of Magnet Overheating}

This section discusses potential ways to postpone degaussing of the samariumcobalt magnets, which occurs at $300{ }^{\circ} \mathrm{C}$. Unless stated otherwise, all models created for this section use the same initial and boundary conditions as the thermal model that was validated in chapter 4 .

\subsection{Model A: Isolate the Keeper Electrode}

One simple way to postpone the degaussing effects of overheating is to provide more thermal insulation to the keeper electrode. During the heating phase of the thrusters operation, the interior of the keeper electrode must be heated to temperatures that allow for plasma formation. Much of this heat is conducted through the electrode, and either radiated onto the thruster or conducted through the Macor isolation sheath. While creating the model that was presented in the previous chapter, it was observed that the Macor isolation sheath is a great thermal insulator and shunts the heat transfer due to conduction and radiation.

During testing, MiXI was shut down before one of the eight thermocouples recorded a temperature of $300{ }^{\circ} \mathrm{C}$. Figure 3.10 shows that Thermocouple 8 (TC8) is the first thermocouple to reach the $300{ }^{\circ} \mathrm{C}$ threshold. Figure 3.10 also tells us that TC8 is at nearly $250{ }^{\circ} \mathrm{C}$ at the time the hollow cathode is ignited. It was hypothesized that the recorded temperature of TC8 is primarily due to radiation 
from the surface of the electrode and that the temperature of the magnet locations is much less than $300^{\circ} \mathrm{C}$. However, those operating MiXI chose to error on the side of caution and shut down the cathode.

The heat that was introduced from the cathode heating phase effectively reduced MiXI-CP-V3's operating time. If the exterior of the keeper electrode were thermally insulated, then MiXI's temperature profile at the time of cathode ignition would be lower, increasing the overall operation time of the thruster.

\subsubsection{Mesh Generation and Model Set Up}

The low thermal conductivity of Macor makes it a good thermal insulator in addition to being an excellent electrical insulator. In order to thermally insulate the keeper electrode from the rest of MiXI, the cathode isolation sheath was increased in both length and thickness. The length of the isolation sheath was increased to $51 \mathrm{~mm}$, long enough to cover the entire cathode. The thickness of the sheath was also increased so that its outer diameter nearly touches the lower ring of magnets. A close up model of this geometry is shown in fig. 5.1. The size of the mesh is 113,747 nodes, 112,000 cells, 225,798 faces. 


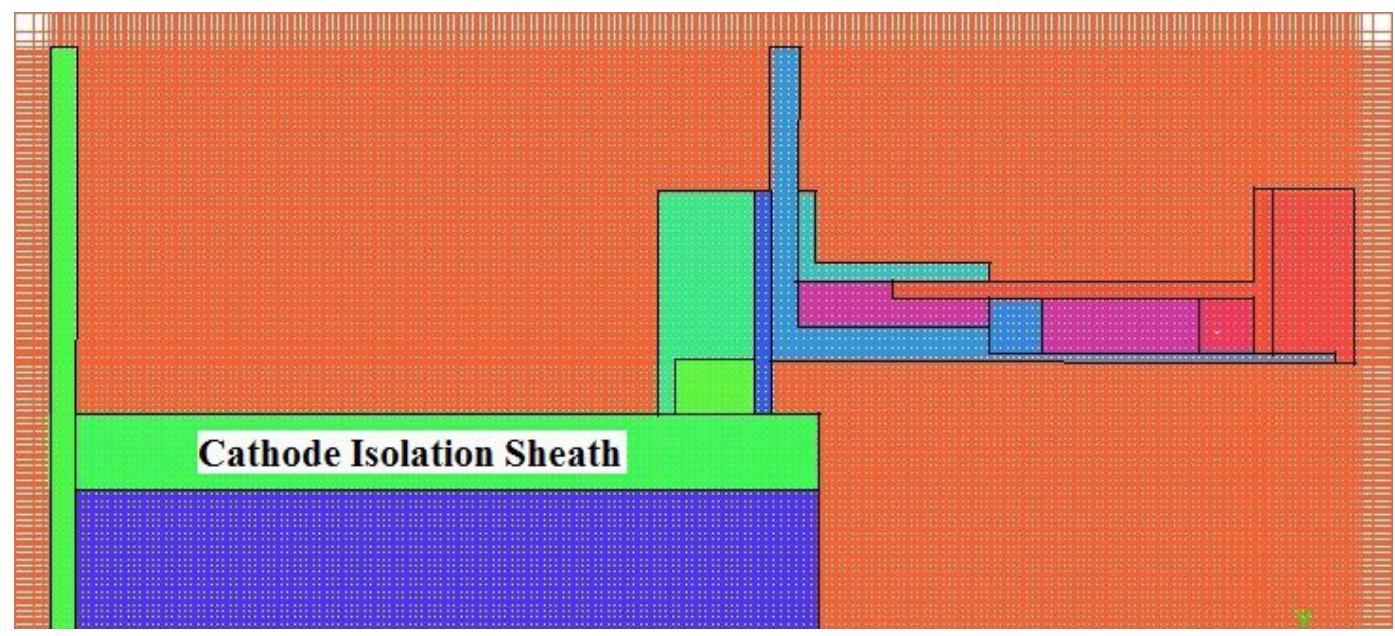

Figure 5.1: The mesh of the expanded keeper isolator.

The boundary conditions of this thermal model are nearly identical to the boundary conditions of the model presented in chapter 4 . Unlike the original model presented in chapter 4 , it was assumed that this model had no gap between the exterior of the isolator and the lower magnet ring. The thermal contact resistance between the magnets and the isolator was assumed to be the same as the contact resistance between the isolator and the magnet-mount and magnetshield.

\subsubsection{Results}

This is a solution that is unique to MiXI-CP-V3 because it is currently the only version of MiXI that utilizes a hollow cathode. However, this solution should be applicable to all MiXI's should hollow cathodes become more common in miniature ion thrusters.

Figure 5.2 depicts the temperature profile of MiXI at the time the cathode was ignited, 22.5 minutes into operation. 


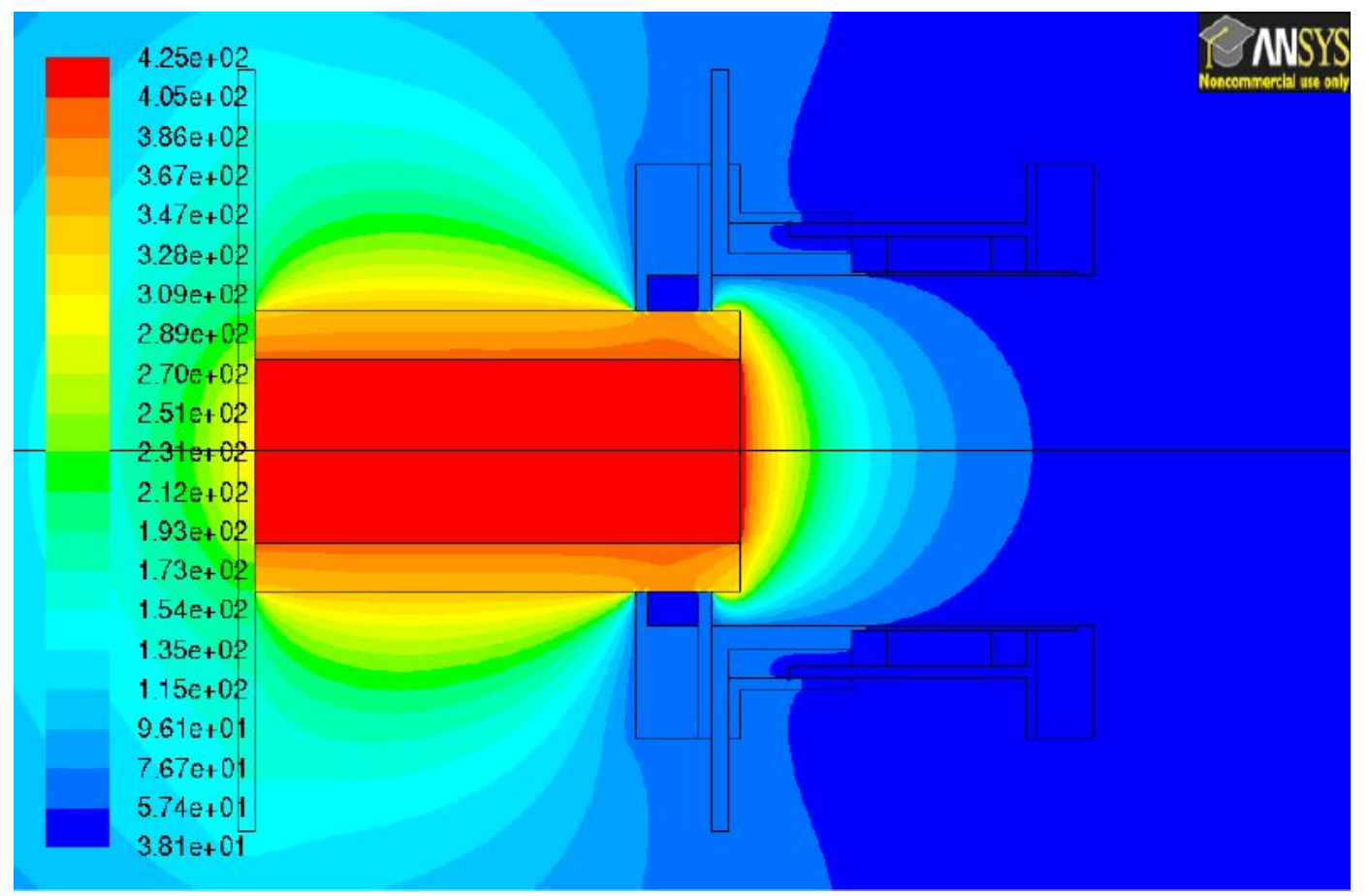

Figure 5.2: Contour plot showing the temperature profile of MiXI if the isolation sheath were extended.

Figure 5.3 depicts the temperature profile of MiXI at the time the cathode was shut down, 22.5 minutes into operation. 


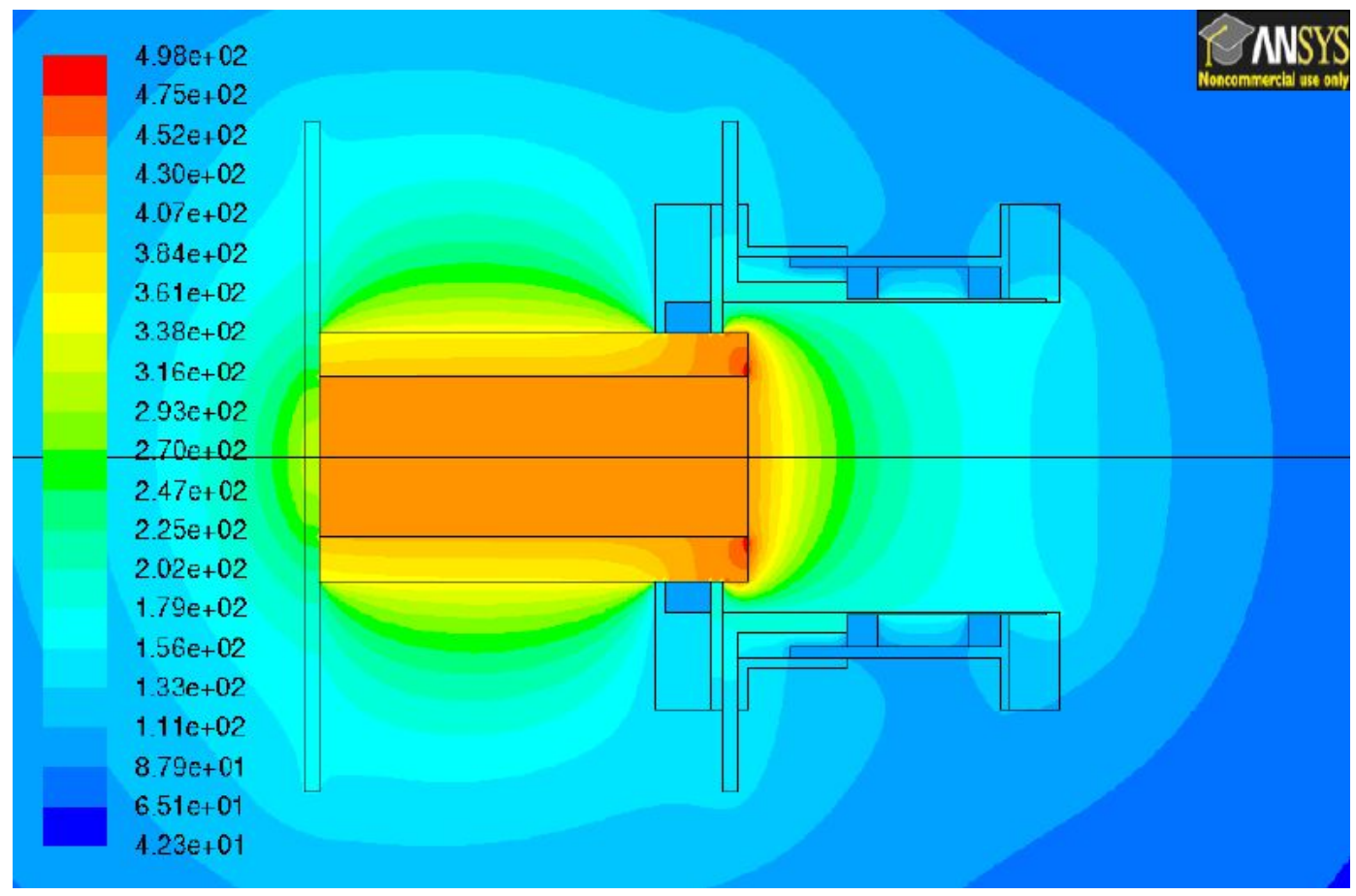

Figure 5.3: Contour plot showing the temperature profile of MiXI if the isolation sheath were extended.

Table 5.1 compares temperatures at thermocouples 1-5 for the model with the larger isolation sheath vs the original model. The results shown in table 5.1, fig. 5.2, and fig. 5.3 indicate that extending the cathode isolation sheath to cover the entire electrode reduces the temperature by as much as $11{ }^{\circ} \mathrm{C}$ in thermocouple locations closest to the keeper electrode. This shows a slight improvement over the original design, but not a very significant one. 
Table 5.1: Temperatures at thermocouple locations as predicted by the original theoretical model, and the model that featured the extended isolation sheath (New).

\begin{tabular}{|c|c|c|c|c|c|c|c|c|}
\hline Time (min) & \multicolumn{2}{|c|}{10} & \multicolumn{2}{c|}{16.5} & \multicolumn{2}{c|}{20} & \multicolumn{2}{c|}{22.5} \\
\hline & Orig & New & Orig & New & Orig & New & Orig & New \\
\hline TC1 $\left({ }^{\circ} \mathrm{C}\right)$ & 36.3 & 35.8 & 47.1 & 44.6 & 77.0 & 73.7 & 119.8 & 115.5 \\
\hline TC2 $\left({ }^{\circ} \mathrm{C}\right)$ & 37.5 & 36.6 & 49.3 & 45.8 & 72.9 & 67.4 & 112.7 & 104.5 \\
\hline TC3 $\left({ }^{\circ} \mathrm{C}\right)$ & 44.9 & 42.4 & 61.7 & 54.2 & 90.6 & 81.1 & 134.6 & 123.1 \\
\hline TC4 $\left({ }^{\circ} \mathrm{C}\right)$ & 49.2 & 45.7 & 68.8 & 58.8 & 105.5 & 93.7 & 155.2 & 141.9 \\
\hline TC5 $\left({ }^{\circ} \mathrm{C}\right)$ & 51.5 & 48.4 & 71.0 & 61.6 & 105.0 & 93.7 & 153.7 & 140.4 \\
\hline
\end{tabular}

\subsection{Model B: Titanium Thruster}

Another possibility for buying the thruster more time to operate is to make it out of materials with a lower thermal conductivity. The anode and grids of ion thrusters are typically made of stainless steel, titanium, graphite, or molybdenum; these materials are chosen because they are resistant to ion bombardment but they have a relatively high thermal conductivity. If the anode were made of a material of lower thermal conductivity, then magnet degaussing might be postponed. Using titanium on a thruster has the added benefit of reducing the thruster's overall mass due to the fact that titanium is much less dense than steel. This is very beneficial for flight thrusters that will be flown in geosynchronous orbits or interplanetary trajectories. Titanium parts are generally not allowed on low-earth orbit missions because they will survive reentry and potentially cause damage or injury on the ground.

In order to test this theory, the thermal model was run with two different grades of titanium alloy rather than stainless steel. The titanium alloys that were used are ASTM Grade 9 and IMI 685. The properties of these materials are shown in table 5.2. The model set up and the results are discussed below. 
Table 5.2: Thermal properties of titanium alloys $[26,25]$.

\begin{tabular}{|c|c|c|c|}
\hline Material & $\rho\left(\frac{\mathrm{kg}}{\mathrm{m}^{3}}\right)$ & $C_{P}$ & $k\left(\frac{\mathrm{W}}{\mathrm{m}-K}\right)$ \\
\hline ASTM Grade 9 & 4480 & 520 & 7.6 \\
\hline IMI 685 & 4450 & 520 & 4.8 \\
\hline
\end{tabular}

\subsubsection{Mesh Generation and Model Set Up}

The mesh that was used in this thermal model is identical to the mesh that was validated in chapter 4 . Once the mesh was read into Fluent, the same initial and boundary conditions were applied. The material properties of ASTM Grade 9, or IMI 685 titanium alloy were specified and applied to the anode and magnet shield. Unlike the properties for stainless steel, the thermal conductivity for the titanium was assumed to be constant. Also, the thermal contact resistances between each part were not changed.

\subsubsection{Results}

\section{ASME Grade 9 Titanium Alloy}

Figure 5.4 depicts a contour plot of MiXI's thermal environment after 6 minutes of cathode operation and 22.5 minutes from the time the heater was initiated. 


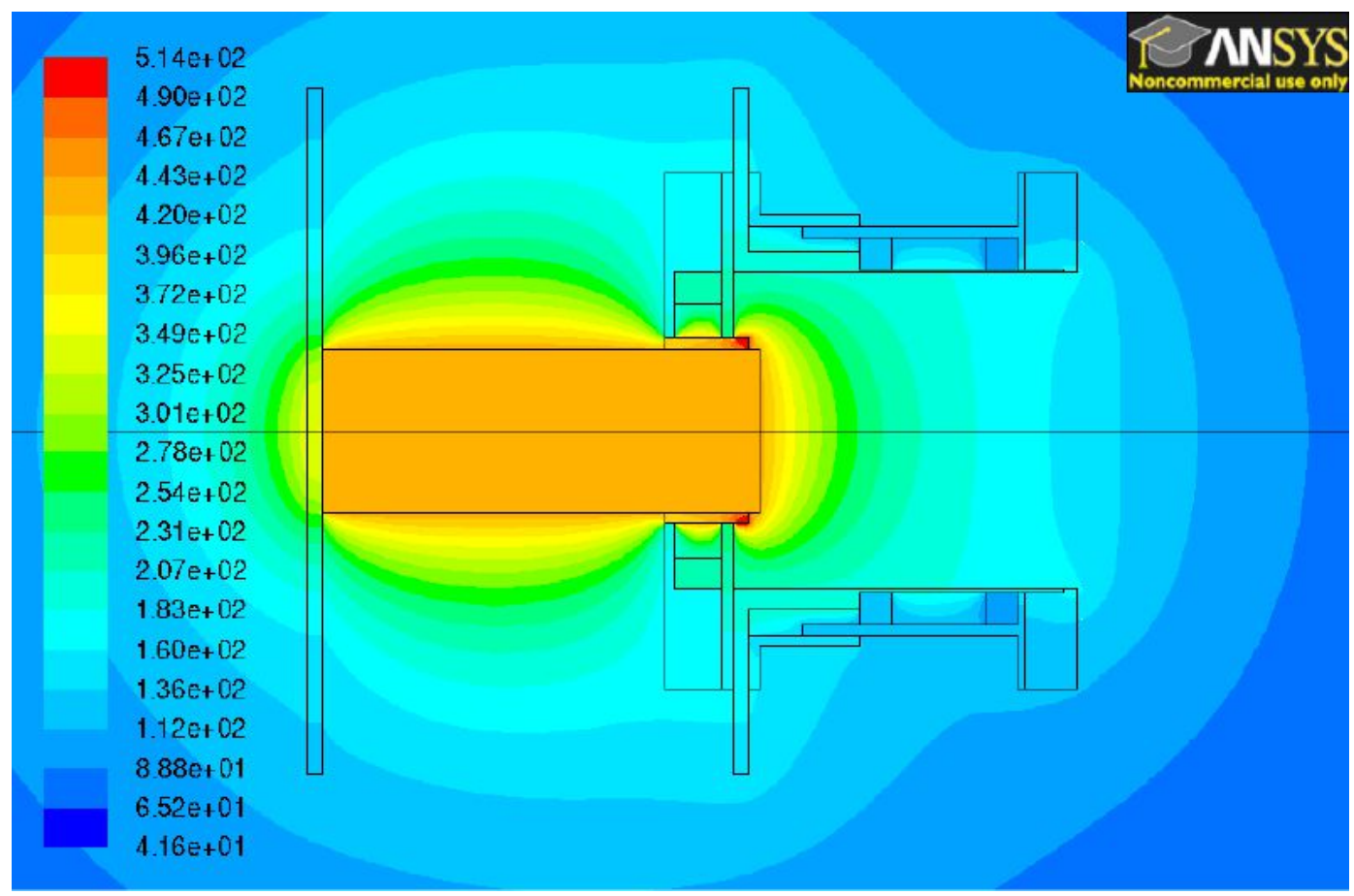

Figure 5.4: Contour plot showing the temperature profile of MiXI if it were constructed of IMI 685 titanium alloy.

Table 5.3 compares temperatures at thermocouples 1-5 for the ASTM Grade 9 model vs the original model. The results from this table indicate that using ASTM Grade 9 titanium will result in sightly higher temperatures at the thermocouple locations at the end of thruster operations. This is counter to the original logic of using titanium, and defeats the purpose of using titanium on the thruster. The behavior observed in this thermal model was unexpected and it is suspected that it may have to do with the thermal diffusivity of the material. Thermal diffusivity is present in the transient term of the heat equation and relates the conductivity, heat capacity, and density of the material. Because only conductivity was considered in the selection of this material, the other material properties may have offset the benefit of a low thermal conductivity. To determine the true cause of the unexpected thermal behavior of ASTM 9, further investigation is required. 
Table 5.3: Temperatures at thermocouple locations as predicted by the original theoretical model, and the model that featured ASTM Grade 9 titanium alloy (New).

\begin{tabular}{|c|c|c|c|c|c|c|c|c|}
\hline Time (min) & \multicolumn{2}{|c|}{10} & \multicolumn{2}{c|}{16.5} & \multicolumn{2}{c|}{20} & \multicolumn{2}{c|}{22.5} \\
\hline & Orig & New & Orig & New & Orig & New & Orig & New \\
\hline TC1 $\left({ }^{\circ} \mathrm{C}\right)$ & 36.3 & 37.5 & 47.1 & 48.9 & 77.0 & 81.2 & 119.8 & 124.4 \\
\hline TC2 $\left({ }^{\circ} \mathrm{C}\right)$ & 37.5 & 40.8 & 49.3 & 54.2 & 72.9 & 79.4 & 112.7 & 118.1 \\
\hline TC3 $\left({ }^{\circ} \mathrm{C}\right)$ & 44.9 & 50.3 & 61.7 & 68.5 & 90.6 & 96.1 & 134.6 & 135.5 \\
\hline TC4 $\left({ }^{\circ} \mathrm{C}\right)$ & 49.2 & 57.5 & 68.8 & 79.2 & 105.5 & 114.5 & 155.2 & 159.9 \\
\hline TC5 $\left({ }^{\circ} \mathrm{C}\right)$ & 51.5 & 58.3 & 71.0 & 79.6 & 105.0 & 109.5 & 153.7 & 151.5 \\
\hline
\end{tabular}

\section{IMI 685 Titanium Alloy}

Figure 5.4 depicts a contour plot of MiXI's thermal environment after 6 minutes of cathode operation and 22.5 minutes from the time the heater was initiated.

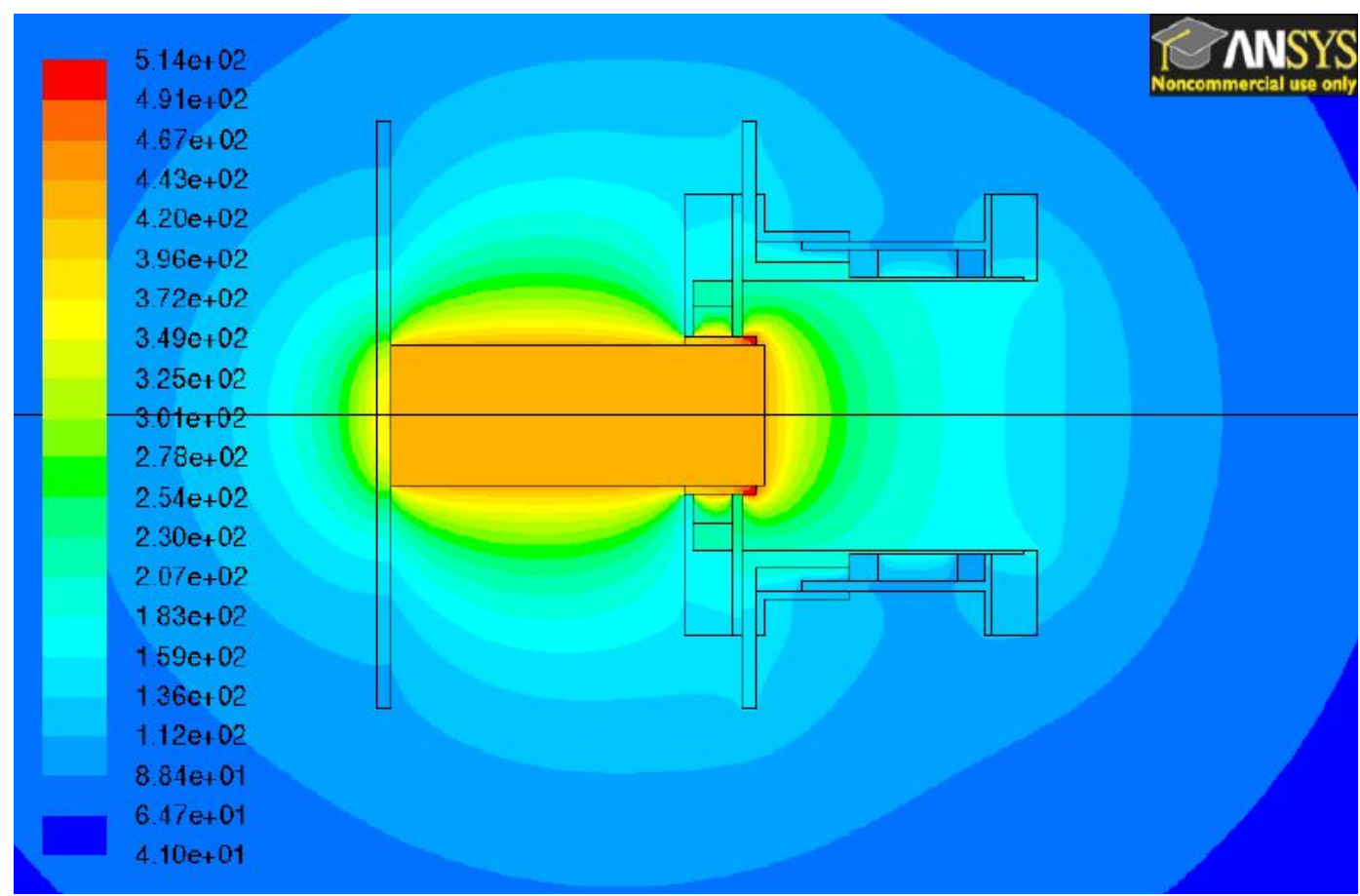

Figure 5.5: Contour plot showing the temperature profile of MiXI if it were constructed of IMI 685 titanium alloy. 
Table 5.4 compares temperatures at thermocouples 1-5 for the IMI 685 model vs the original model. Like with ASME Grade 9, the temperature at the end of thruster firing is either about the same or higher than what was predicted with the stainless steel model.

Table 5.4: Temperatures at thermocouple locations as predicted by the original theoretical model, and the model that featured IMI 685 titanium alloy.

\begin{tabular}{|c|c|c|c|c|c|c|c|c|}
\hline Time (min) & \multicolumn{2}{|c|}{10} & \multicolumn{2}{c|}{16.5} & \multicolumn{2}{c|}{20} & \multicolumn{2}{c|}{22.5} \\
\hline & Orig & IMI & Orig & IMI & Orig & IMI & Orig & IMI \\
\hline TC 1 ( $\left.{ }^{\circ} \mathrm{C}\right)$ & 36.3 & 36.0 & 47.1 & 45.6 & 77.0 & 77.1 & 119.8 & 119.8 \\
\hline TC 2 $\left({ }^{\circ} \mathrm{C}\right)$ & 37.5 & 40.3 & 49.3 & 53.1 & 72.9 & 75.1 & 112.7 & 110.8 \\
\hline TC 3 $\left({ }^{\circ} \mathrm{C}\right)$ & 44.9 & 48.4 & 61.7 & 65.7 & 90.6 & 87.7 & 134.6 & 121.9 \\
\hline TC 4 $\left({ }^{\circ} \mathrm{C}\right)$ & 49.2 & 56.9 & 68.8 & 78.5 & 105.5 & 107.4 & 155.2 & 147.5 \\
\hline TC 5 $\left({ }^{\circ} \mathrm{C}\right)$ & 51.5 & 56.4 & 71.0 & 77.0 & 105.0 & 99.2 & 153.7 & 133.8 \\
\hline
\end{tabular}

Both models of the titanium thrusters showed higher than expected temperatures at the end of thruster operations. This shows that making the thruster out of a less thermally conductive material may not postpone the onset of magnet degaussing, and therefore, the continued use of stainless steel for is recommended for laboratory thrusters.

\subsection{Model C: Insulative Coatings}

One commonly proposed solution to postpone the onset of magnet degaussing involves increasing the thickness of the anode. Obviously, having more material for heat to propagate through means that the magnets will not reach their degaussing temperature as quickly. The major downside to this solution is that it distances the magnets from the plasma, reducing the effectiveness of the magnets and defeating the purpose of thickening the anode. 
An alternative solution that seemed to be promising involved coating the exterior of the anode with a thin layer of insulation material. This material would be only 1-2 mm thick and provide a buffer between the anode and the magnets. Before any of these materials could be modeled, many problems arose that ruled out their applicability to MiXI.

Many epoxies, (such as DC 93-500 Eccobond 285) were looked at because of their low thermal conductivity $\left(<1 \frac{W}{m-K}\right)$, however, they have a melting point (300-320 ${ }^{\circ} \mathrm{C}$ ) that is comparable to the degaussing temperature of the SmCo magnets on MiXI[22]. Polyether ether ketone (PEEK) was also considered as an insulator because it's ability to withstand high-vacuum environments and its use as a thermal insulator. Unfortunately, PEEK has the same problem as the epoxies that were considered; PEEK melts at a temperature of $343{ }^{\circ} \mathrm{C}$ and, if incorporated into MiXI, might melt[22].

Thermal interface materials were also considered, however, these are intended to increase thermal contact conductance between interfacing surfaces. This would not have postponed the onset of magnetic degaussing, therefore, using these materials would be pointless.

After finding too many flaws with insulating the anode or increasing its thickness, it was decided that the implementation of these methods is not practical. As a result of this impracticality, coating the anode in an insulative material or increasing the anode thickness was not modeled. Additionally, it is not recommended that any future users of MiXI attempt to incorporate these methods into the thruster. 


\section{Conclusion}

Cal Poly's MiXI-CP-V3 was operated in a vacuum chamber and temperature data was collected at eight points around the thruster. The temperature data was then used to validate a transient, two dimensional thermal model of MiXI that has been created in ANSYS Fluent. At four of the eight thermocouple locations, the model predicted temperatures within $11{ }^{\circ} \mathrm{C}$ of the empirical data. At the three thermocouple locations that were perpendicular to the keeper electrode, the model indicated a temperature about $70{ }^{\circ} \mathrm{C}$ less than the empirical data. The empirical temperatures recorded at these locations is most affected by radiation from the keeper electrode onto the surface of the thruster. The model's ability to simulate this radiation phenomenon was quite poor, therefore, the model's temperature predictions at these locations were tossed out. The temperature data of the remaining thermocouples closely followed the trends of the empirical data.

The model indicates that MiXI is being shut down prematurely during testing. The tests conducted with MiXI were shut down when one of the thermocouples reached about $290{ }^{\circ} \mathrm{C}$. The first thermocouple to reach this temperature was the thermocouple that was mostly being affected by the radiation emitting from the keeper electrode. The model indicates that the temperature of the hottest magnet may be as much as $100{ }^{\circ} \mathrm{C}$ less than the temperature of the hottest thermocouple. 
Two new thermal models were created and used to assess two proposed methods of postponing the effects of magnet degaussing. One model simulated the effects of shielding the entire keeper electrode with the cathode isolation sheath, and one model tested the effects of using titanium for thruster parts rather than steel. Shielding the cathode with a large isolation sheath was shown to reduce the predicted temperature at the thermocouples by as much as $10^{\circ} \mathrm{C}$ after 22.5 minutes of operating time. The models showed that building a new thruster out of titanium parts would have little effect on MiXI's transient thermal environment.

The models and research conducted for this thesis all indicate that attempting to postpone magnetic degaussing will have little effect on thruster operating time. Making the thruster with titanium alloy will make it lighter but will be very expensive and not postpone magnetic degaussing. Shielding MiXI from the keeper electrode may only allow the thruster to be operated for i 1 minute longer than it is currently capable of operating. The complete prevention of magnetic degaussing may be possible but will likely require an active heat sink. The issue of preventing magnetic degaussing should be re-examined when a better thermal model is developed.

\subsection{Recommendations}

This project was ultimately unable to find the elusive "silver bullet" solution for MiXI's overheating problem. However, the next person to operate MiXI-CP-V3 may want to increase the size of the cathode isolation sheath to completely cover the keeper electrode. Insulating the entire keeper electrode will not only shunt the heat transfer to MiXI, but it may have the added benefit of preventing heat from escaping the electrode during the heating phase. This would increase the 
rate of temperature increase within the electrode, thus allowing cathode ignition to occur a few minutes earlier. Making a new thruster out of titanium alloys will do little to postpone the onset of magnet degaussing so the laboratory version of MiXI is better off in its current form. However, titanium is much less dense than stainless steel and therefore a thruster made of titanium will be much less massive than a steel thruster. For this reason, titanium alloys may be considered for use in flight thrusters.

During testing, the thruster was stopped prematurely because TC8 was nearing the $300{ }^{\circ} \mathrm{C}$ limit. At the time, it was hypothesized that this was the radiated temperature at that thermocouple and the temperature of the magnets is less than $300^{\circ} \mathrm{C}$; however, it was decided to error on the side of caution and stop the test. The thermal model shows that thermocouples placed at locations 1-4 (see chapters 3 and 4) will be less affected by radiation and therefore, provide a reading that is closer to the temperature of their nearest magnets. In its current form, MiXI-CP-V3 is capable of operating for several minutes longer than has been operated in the past. However, the exact point at which the thruster reaches steady state cannot be known because of the way in which the heat exchange was modeled (using a UDF with a simple dT/dt).

Although not studied in this thesis, it may be beneficial to actively cool the walls of the vacuum chamber. During thruster operation, the thruster imparts a heat flux onto the walls of the chamber via radiation heat transfer. If the walls of the chamber were constantly cooled, they would take the heat being radiated onto them and effectively reduce the steady-state operating temperature of the thruster. It would be difficult to accomplish this in the MAX chamber at Cal Poly, but it may be possible to cool the walls of one of the other vacuum chambers. 


\subsection{Future Work}

This projects goal was to determine a design change that would allow MiXI to operate for longer durations than what is currently possible. The only change that shows any signs of promise involves extending the cathode isolation sheath to cover the entire cathode. The next student to operate MiXI-CP-V3 may want to increase the size of the isolation sheath;but this change will not significantly increase MiXI's operating time. However, having an isolation sheath that covered the entire keeper electrode might also mitigate the arcing between the keeper electrode and MiXI, which caused the spikes seen in the thermocouple data in fig. 3.9.

Numerical thermal modeling is quite a challenging task, and the models shown in this thesis have room for improvement. Two questions that have been left unanswered are the prediction of when the thruster reaches steady-state thermal conditions and the temperature of the steady-state environment. These are questions that could not be answered using this thermal model because of the way it was modeled. Should someone chose to create a new thermal model, it is recommended that they utilize Fluent's MHD module to model plasma flow within the chamber. If modeling magnetohydrodynamics is not feasible, a next best option would be to determine the power deposition onto the anode during thruster operations. This is the method used by Coleman Younger when developing a thermal model of MiXI-CP-V1 and by Mr. Van Noord for the thermal model of NASA's NEXT thruster[15, 21]. These techniques would be better suited to model long duration burns of MiXI, unlike the model created for this thesis which relies on a temperature boundary condition that was measured empirically.

The next thermal model of MiXI should be three dimensional model fo the 
MiXI-vacuum chamber environment and include more comprehensive radiation analysis. The P-1 radiation model provides reasonable results but does not adequately model the temperature at a surface due to radiation. A three dimensional model would not be able to use axis-symmetric boundary conditions and therefore would be able to utilize Fluent's Surface to Surface (S2S) radiation model. A three dimensional model that models plasm flow or power deposition due to plasma would be better suited to model improvements for MiXI.

The test data collected for this thesis is quite good and could be useful in the future. The testing data that was collected for this thesis was shown to be very consistent and repeatable, and should someone in the future wish to create a more comprehensive model, the data from this thesis could be used for validation. However, if someone were to repeat this experiment it is recommended that they place thermocouples on both the top and bottom of the thruster. This project assumed symmetry about the center axis of the thruster; that assumption allowed thermocouples to be placed on the upper half of the thruster in the same 2D plane. Several thermocouples should have been placed on the bottom half of the thruster in order to confirm the validity of the axis-symmetric assumption.

Increasing the anode thickness was not considered a viable option for this thesis project because it would affect the magnetic field topography in the chamber. During the final stages of this project, it was brought to the attention of the author that the original magnetic field analysis may be incorrect and MiXI's current magnets may be too strong. If this is the case, then it may be desirable to increase the thickness of the anode so that the magnetic field is weaker and the magnets have increased protection from plasma heating.

One of the biggest hurdles in the way of making MiXI a viable flight thruster is the size of the cathode. MiXI-CP-V3 is unique in that it was the first MiXI 
thruster to utilize a hollow cathode for electron bombardment. This configuration works in a laboratory environment but the current cathode is far too large for a MiXI thruster. Several attempts have been made to scale down hollow cathodes but there currently is no appropriately-sized hollow cathode for MiXI.

This thesis has shown that many passive temperature control methods, such as insulating the keeper electrode, only postpone the onset of magnetic degaussing. While postponement of this phenomenon was the goal of this thesis, it still does not change the fact the magnets will eventually degauss before the thruster's thermal environment reaches steady state. Once a three dimensional thermal model has been developed, another student may use it to model active thermal management systems such as heat pipes or regenerative coolers.

Whomever decides to create the next thermal model of MiXI-CP-V3, it is recommended that they have extensive experience with Fluent, or whichever software program they choose to use. Modeling complex systems in Fluent is daunting process and should not be taken lightly. 


\section{BIBLIOGRAPHY}

[1] Introduction to ansys fluent: Lecture 7, heat transfer modeling, December 2010.

[2] Thermal contact resistance. Online, February 2011.

[3] Macor: Machinable glass ceramic for industrial applications, 2012.

[4] Ni daq chassis, 2013. http://sine.ni.com/nips/cds/view/p/lang/en/nid/207535.

[5] ANSYS, Inc. ANSYS Fluent 12.0 User's Guide, April 2009.

[6] I. B. Celik. Procedure for estimation and reporting of discretization error in cfd applications.

[7] R. E. Conversano, Ryan W.; Wirz. Cubesat lunar mission requiring a miniature ion thruster. Technical report, University of California, Los Angeles, 2012.

[8] D. G. Gilmore. Spacecraft Thermal Control Handbook, volume Volume I: Fundamental Technologies. The Aerospace Press, 2002.

[9] N. Glen. Overview of ion propulsion, December 2008.

[10] K. I. Goebel, Dan M. Fundamentals of Electric Propulsion: Ion and Hall Thrusters. John Wiley \& Sons, Inc., 2008. 
[11] P. C. Hill, Philip. Mechanics and Thermodynamics of Propulsion. AddisonWesly Publishing Company, 2 edition, 1992.

[12] J. Holman. Heat Transfer. McGraw Hill, 10 edition, 2010.

[13] B. L. Incropera, DeWitt. Introduction to Heat Transfer. John Wiley \& Sons, fifth edition edition, 2007.

[14] R. G. Jahn. Physics of Electric Propulsion. Dover Publications, Inc., 2006.

[15] D. W. Knapp. Implementation fo a $1 / 4$ inch hollow cathode into a miniature xenon ion thruster (mixi). Master's thesis, California Polytechnic State University, San Luis Obispo, 2012.

[16] R. D. Knight. Physics For Scientists And Engineers: A Strategic Approach. Pearson, 2005.

[17] J. Lyman Spitzer. Physics of Fully Ionized Gasses. Dover Publications, Inc., 1990.

[18] C. Madhusudana. Thermal Contact Conductance. Mechanical Engineering Series. Springer, 1996.

[19] P. S. McGrail, Scott. Preliminary design of a cylindrical hall effect thruster. Technical report, California Polytechnic State University, San Luis Obispo, 2012.

[20] M. F. Modest. Radiative Heat Transfer. Academic Press, third edition edition, 2013.

[21] J. L. V. Noord. Next ion thruster thermal model. Technical report, NASA, Glenn Research Center, 2010. 
[22] M. E. W. Predmore, Romer E. Materials selection guide. Technical report, NASA Goddard Space Flight Center, Greenbelt, Maryland, August 1990.

[23] L. C. Rodriguez, J. Planet quest: Missions terrestrial planet finder.

[24] B. O. Sutton, George P. Rocket Propulsion Elements. John Wiley \& Sons, Inc., 10 edition, 2010.

[25] B. E. Touloukian, Y.S. Thermophysical Properties of Matter: Specific Heat, Metallic Elements and Alloys, volume Volume 4 of The TPRC Data Series. IFI/Plenum Data Corporation, 1970.

[26] P. R. H. C. K. P. Touloukian, Y.S. Thermophysical Properties of Matter: Thermal Conductivity, Metallic Elements and Alloys, volume Volume 1 of The TPRC Data Series. IFI/Plenum Data Corporation, 1970.

[27] C. T. Younger. Thermal models for a $3 \mathrm{~cm}$ miniature xenon ion thruster (mixi). Master's thesis, California Polytechnic State University, San Luis Obispo, 2010. 


\section{A. User Defined Function}

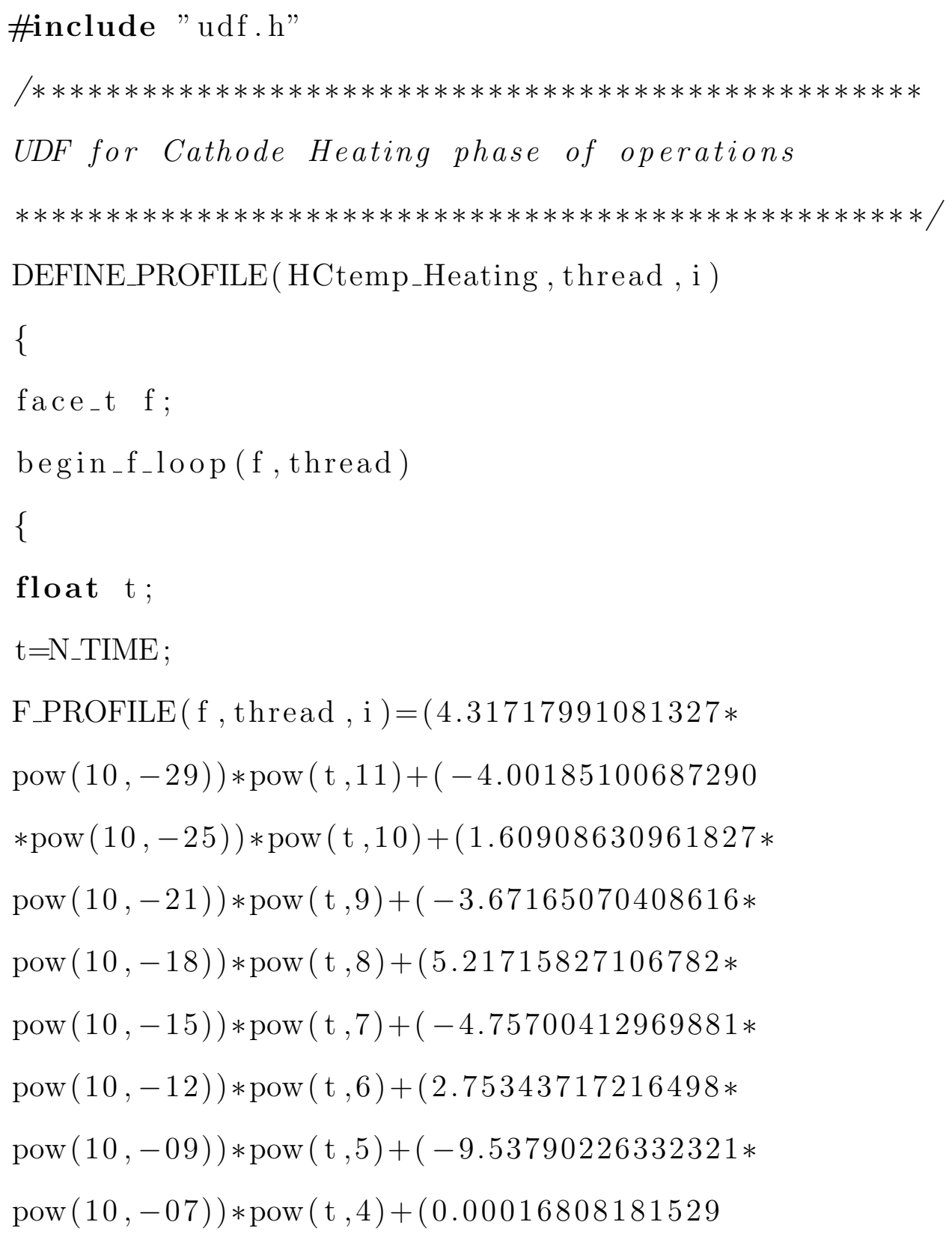




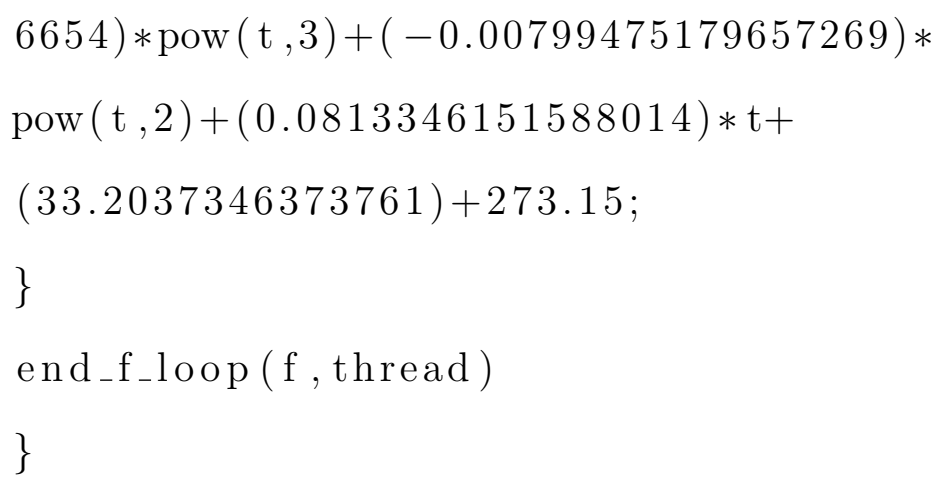




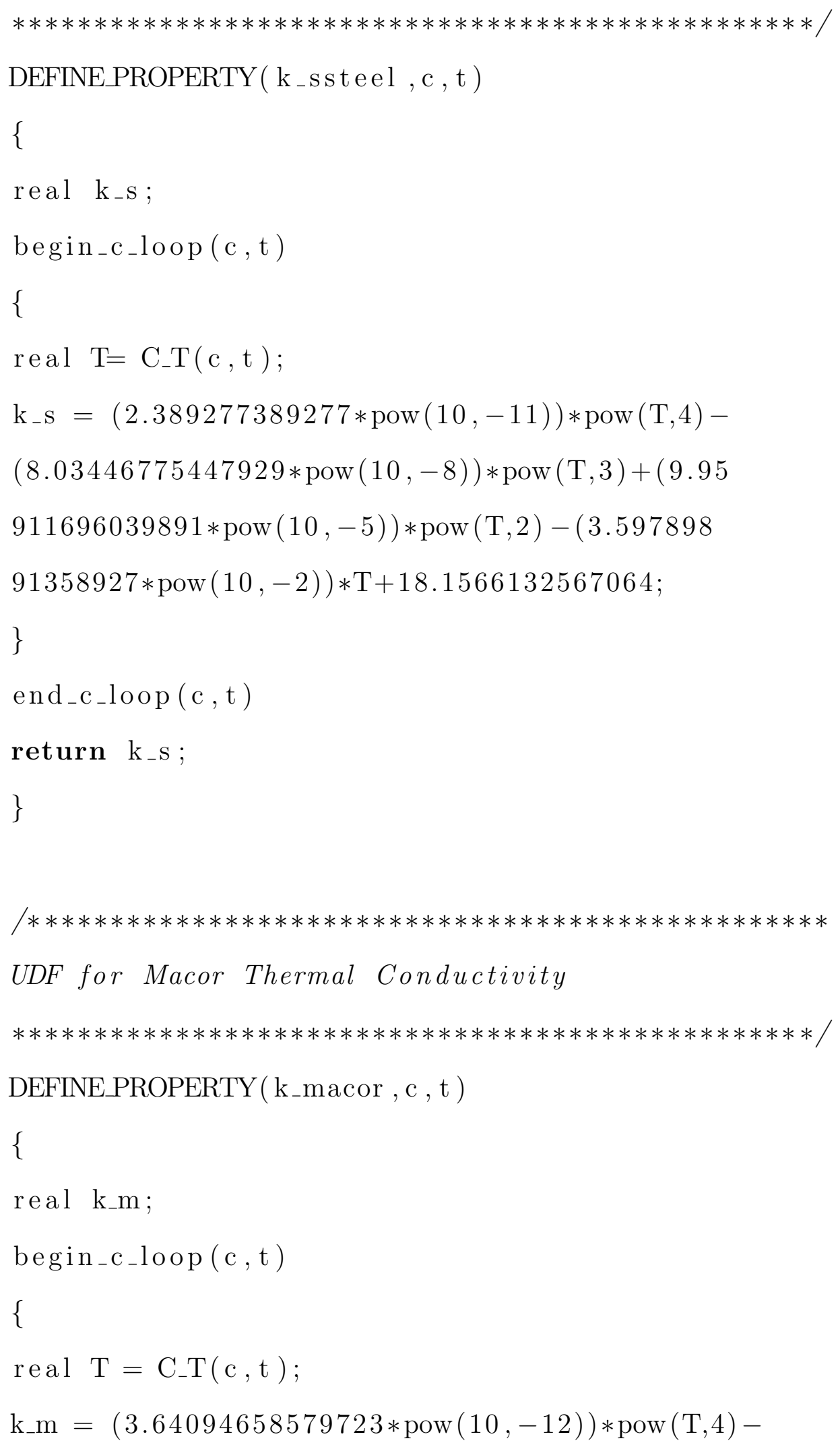




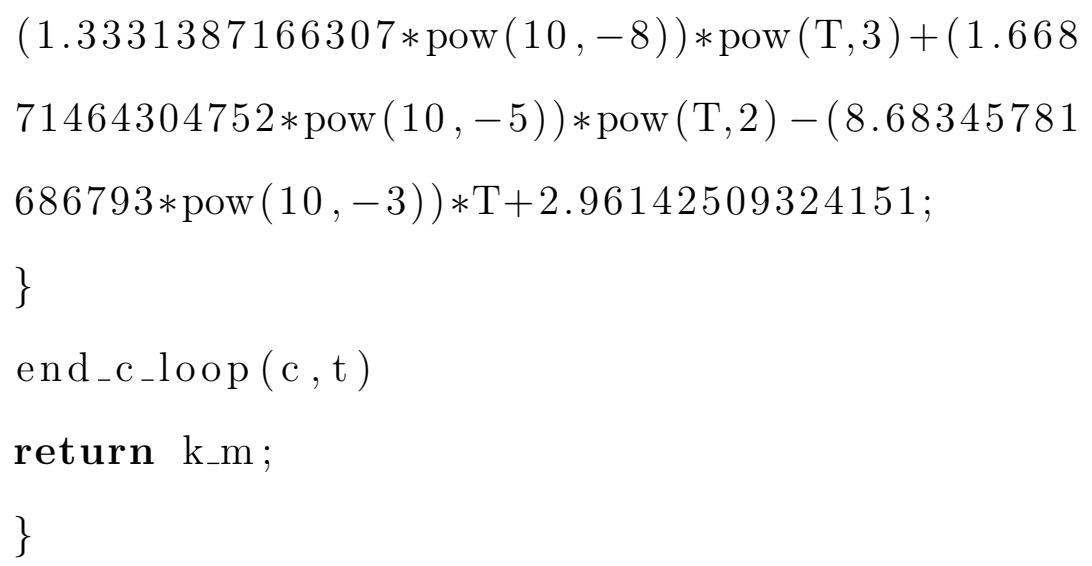




\section{B. Lessons Learned}

This section is intended to help any brave soul that wishes to embark on a project that involves testing MiXI, or modeling in Fluent.

\section{B.1 Testing}

This section discusses many tips and lessons learned while operating MiXI-CPV3 in the Minimum Atmospheric eXperimentation Chamber in Cal Poly's Space Environments Laboratory. This is essentially a list of do's and dont's that I wish I had known when I began the testing phase.

\section{Finding Leaks in the Chamber}

First of all, there is no such thing as a perfect vacuum. Just be aware that every component that exists between the atmosphere and the vacuum will leak slightly. Some components leak more than others but if you're having trouble reaching the desired base pressure, try to reduce the number of valves and feedthroughs in the system (if possible). Caps leak less than electrical or piping feedthroughs, so cap

off as many interfaces as possible. When attaching caps and feedthroughs to the chamber, make sure you clean the surface of the interface with a little acetone. Also, wash the O-rings with water; avoid using acetone because you may damage the O-rings. After all interfaces are clean, apply a few dabs of vacuum grease 
to the O-rings and fasten the feedthrough to the chamber. Use a proper wrench and tighten the feedthroughs as much as you can.

If all of this advice has been tried and the base pressure still cannot be reached, then Cal Poly's ME department has a leak detector that can be used to find leaks. But in my experience, that leak checker is a waste of time. It is much faster and just as effective to simply remove all of the caps and feedthrouhgs, clean them thoroughly with water and acetone, apply a new coating of vacuum grease, and re-attach them.

For the MAX chamber in particular, you can predict the lowest pressure that can be reached with the cryo pump without ever turning on the cryo. Pump the test section of the chamber down with the mechanical pump (give it a good 45 60 mins). As a rule of thumb, if the base pressure achieved in the chamber with the mechanical pump is less than 20 milliTorr, then you're base pressure when using the cryo will be about 1e-6 Torr or less. If you're goal is to operate the hollow cathode in MAX, then do what you can to get the base pressure with the mechanical pump below 20 milliTorr. This will save you lots of time and extend the compressor's life. Also,the base pressure in the MAX chamber has reached as low as 8 milliTorr with the mechanical pump (for those of you that are wondering if it can get below 35 milliTorr, yes it can).

When the propellant system is introduced to the vacuum chamber system, the base pressure will increase due to the unavoidable leaks introduced into the system. If you just barely reach the desired base pressure before the propellant system is set up, you may not attain the same base pressure after the propellant lines are introduced.

When the cryo chamber has been pumped down, toggle the DGAS switch on 
the Ion Gauge to the 'on' position. Let the ion gauge DGAS for 10-15 minutes. The gauge will then provide a more accurate pressure reading.

\section{The Gate Valve}

If you're going to be operating the vacuum chamber with the cryo pump, be sure that any wires or thermocouples are tied-up, away from the gate valve. The gate valve on MAX is like a plate that sits over the cryo-chamber. When the gate valve is open the plate is pushed upward into the test chamber, leaving a 4-5 inch gap between the valve and the chamber interface. If any wires or loose parts are dangling in this area, they can easily fall into the chamber and prevent the gate valve from closing all the way. If this happens, DON'T equalize to atmospheric pressure or you may damage the cryo pump. Simply turn off the compressor and turn on the mechanical pump as you would in a regen cycle (see the procedures in appendix D). Wait until the cryo has warmed to room temperature, turn off the mech pump, vent the chamber, and pull the wires out of the cryo chamber. If something like a bolt or nut has fallen in there, you have no choice but to remove the cryo chamber and invert it until the bolt or nut falls out.

\section{The Compressor}

If the cold head makes a grinding noise then the compressor shuts off after about five seconds of operation, the AC current into the compressor may be out of phase. To check, drag the compressor over to another three-phase power outlet and see if the problem persists. If the compressor works when plugged into another outlet, then there is probably a short somewhere in the MAX vacuum chamber's wiring, which is causing the compressor to get the incorrect phase. 
If the compressor has been on for several minutes, hours, or days and the cold head begins to make a knocking or grinding noise, then the helium in the compressor-cryo system is contaminated and must be replaced. See the compressor's users manual for instructions.

\section{The Hollow Cathode}

When operating the hollow cathode, you'll need to have the keeper power supplied by two sources: the Glasman high-voltage power supply for ignition, and a medium-voltage power supply for nominal operations. Place multiple diodes in between the cathode and each power supply. The diodes protect the power supplies from over powering one another. Having multiple diodes in series will allow you to continue with the test should one of them fail.

Ensure that there is no oxygen in the chamber. Before introducing propellant into the propellant lines, make sure that the 


\section{Plots of Test Data}

This shows all the thermocouple data that was collected during testing plotted as a temperature vs. time. For the sake of brevity, only images are shown. If you'd like the numerical data, please contact the author. It should be noted that the line-plots presented in this report were created from a set of discretized data collected at a rate of one data point per second. Line plots were used in lieu of scatter plots for aesthetic purposes.

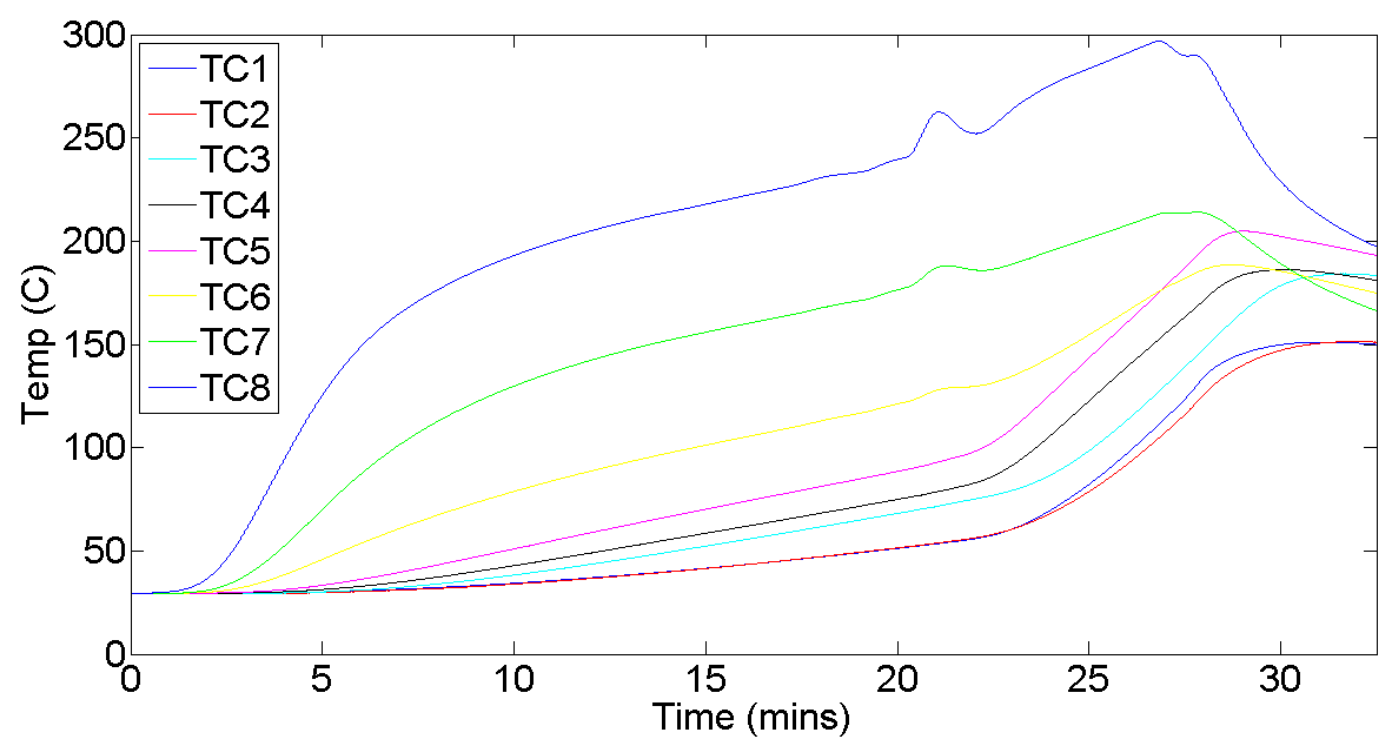

Figure C.1: Test 1. 


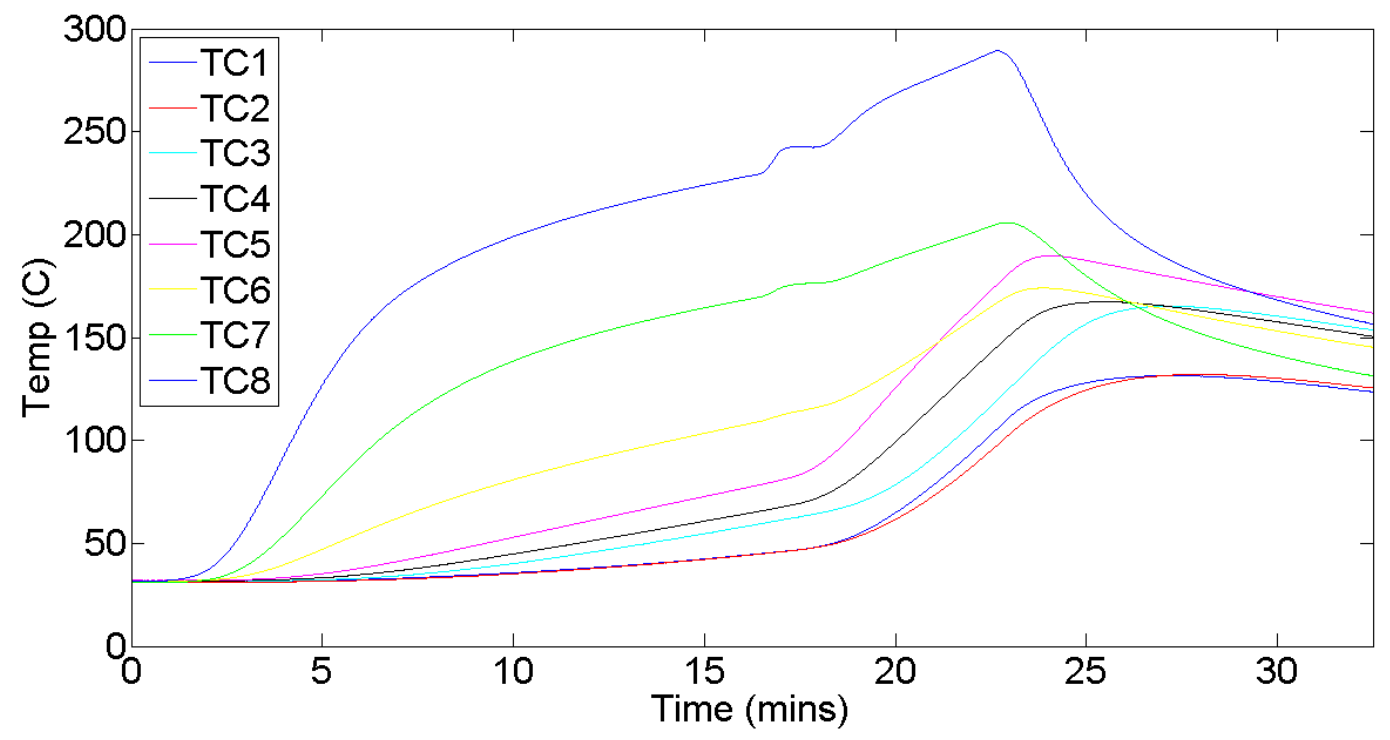

Figure C.2: Test 2.

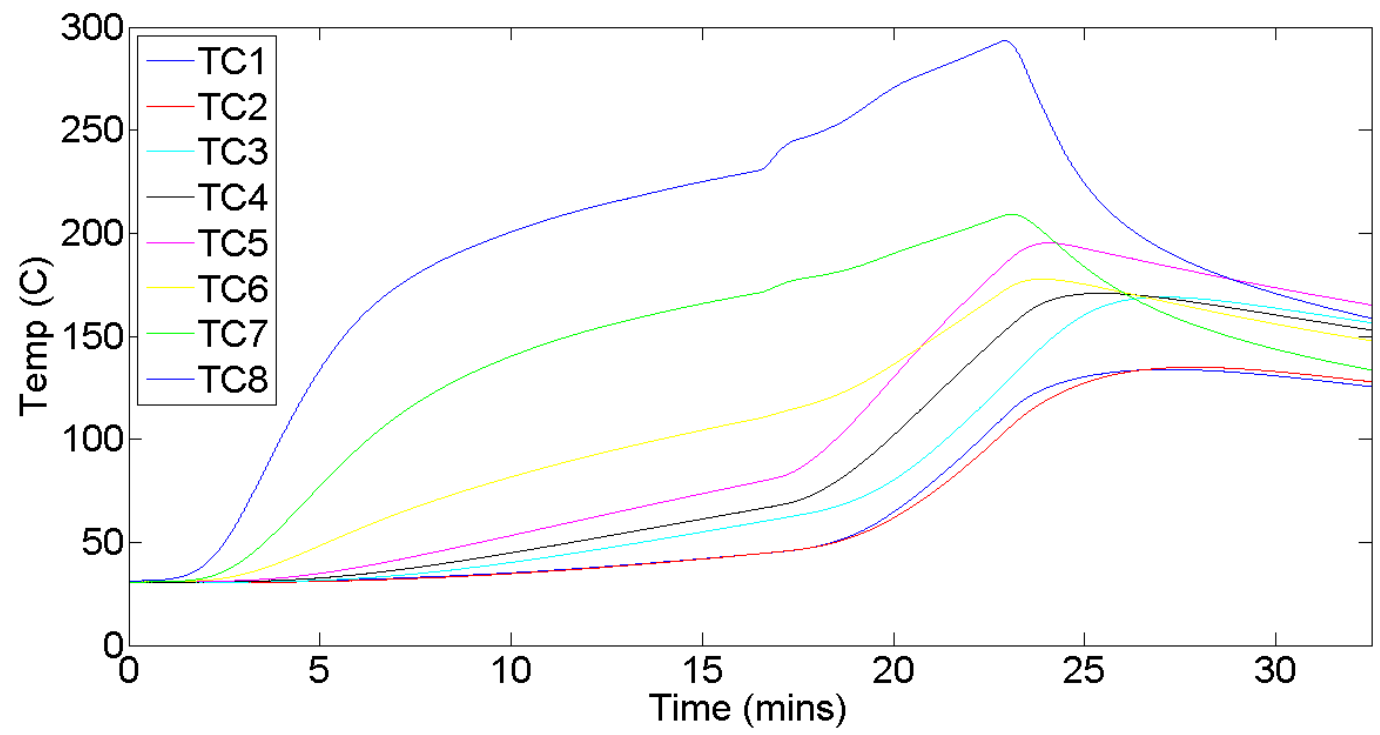

Figure C.3: Test 3. 


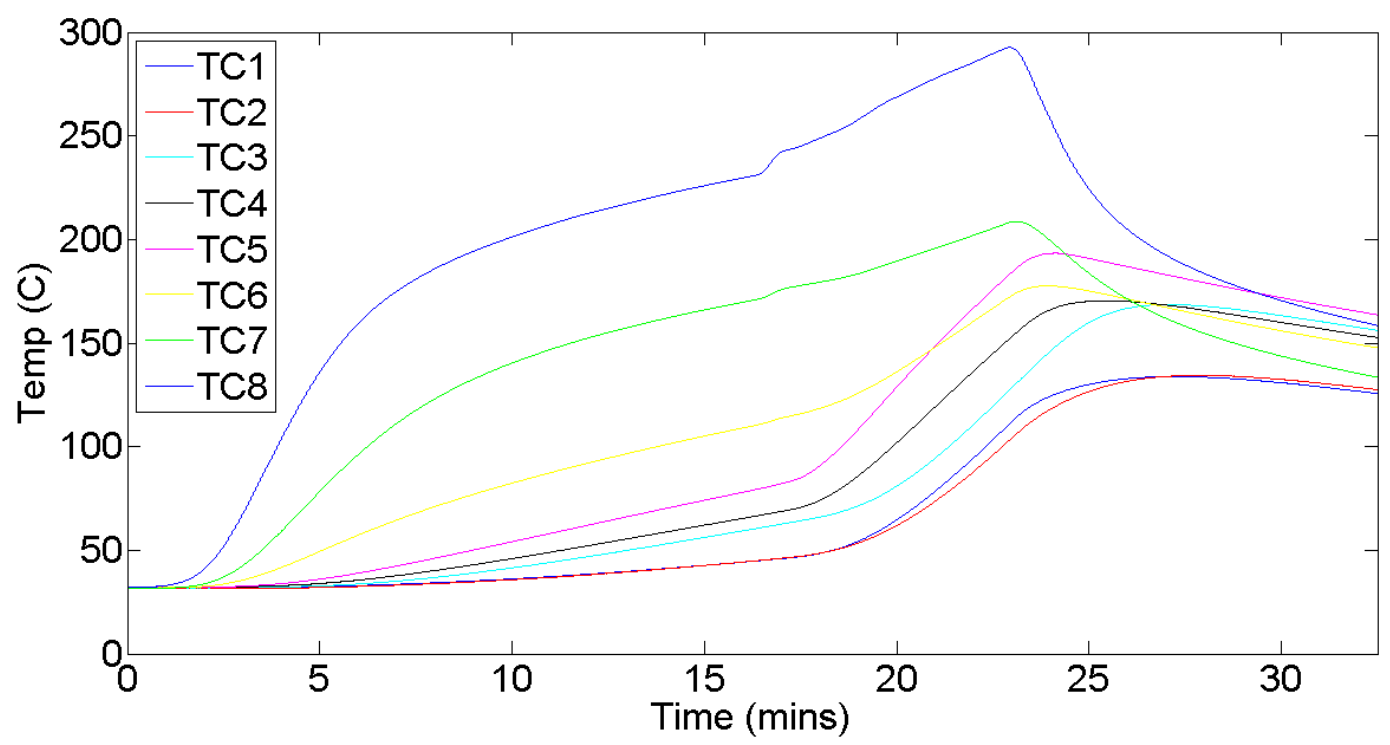

Figure C.4: Test 4.

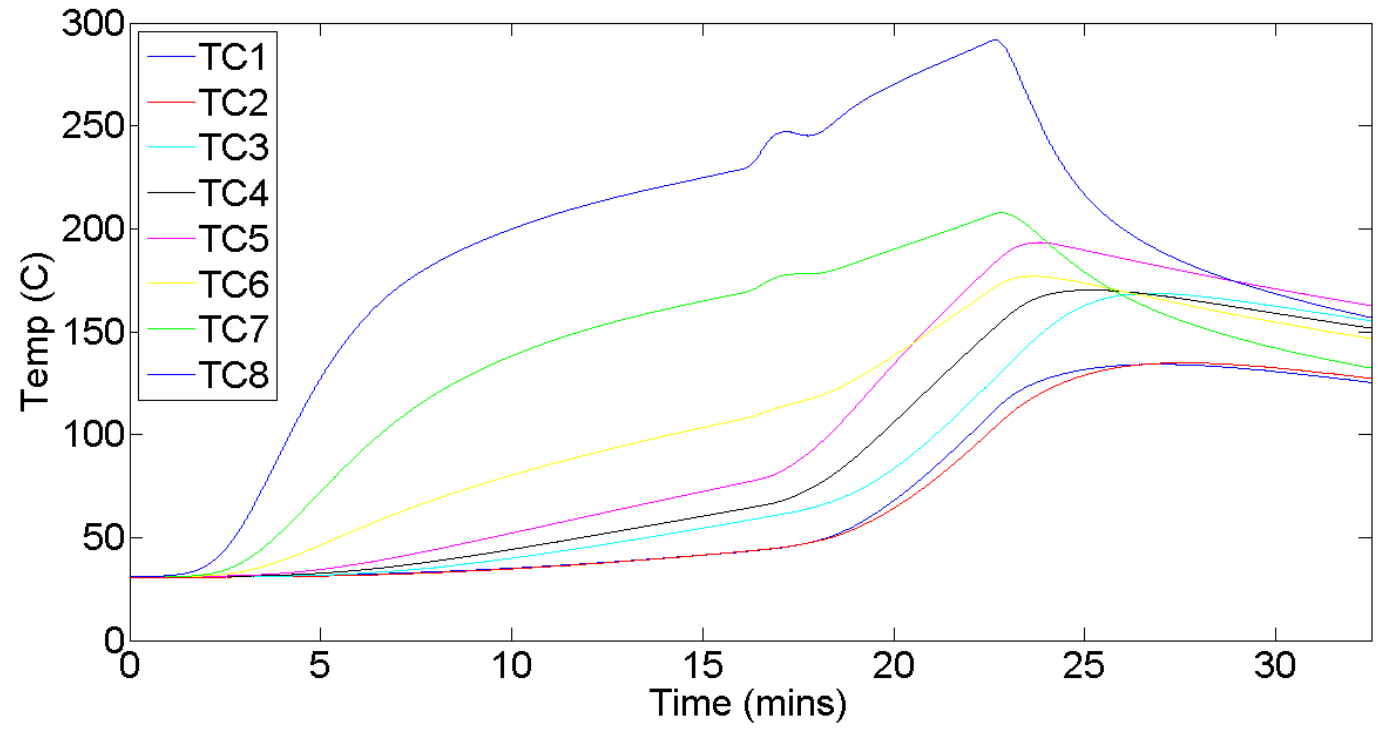

Figure C.5: Test 5. 


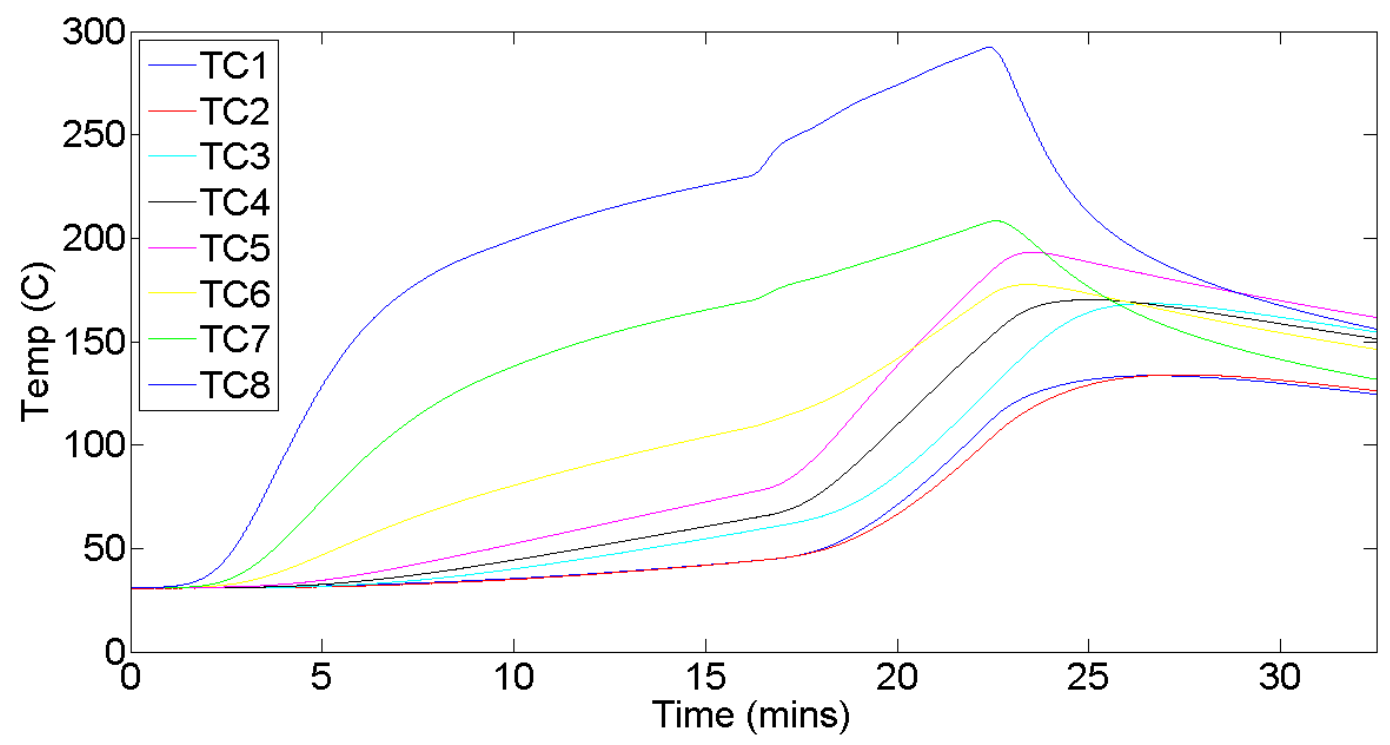

Figure C.6: Test 6.

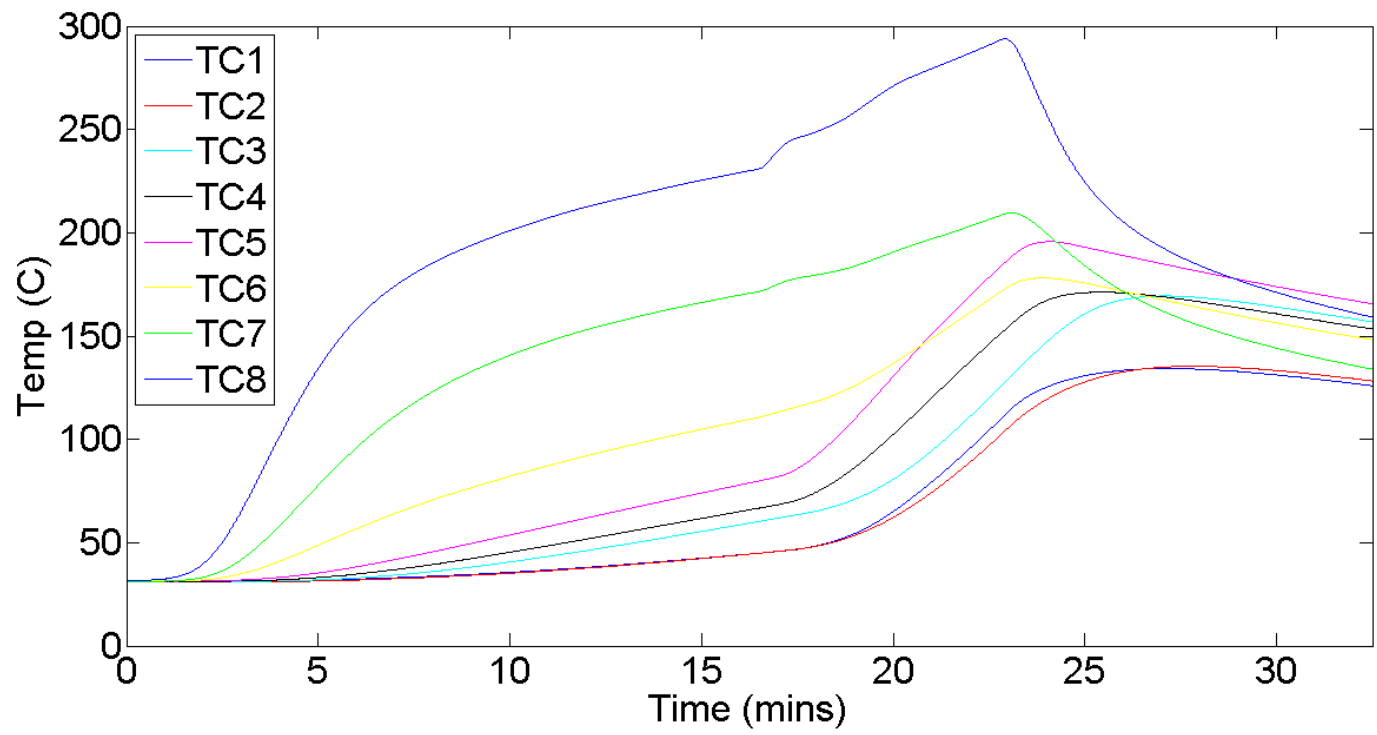

Figure C.7: Test 7. 


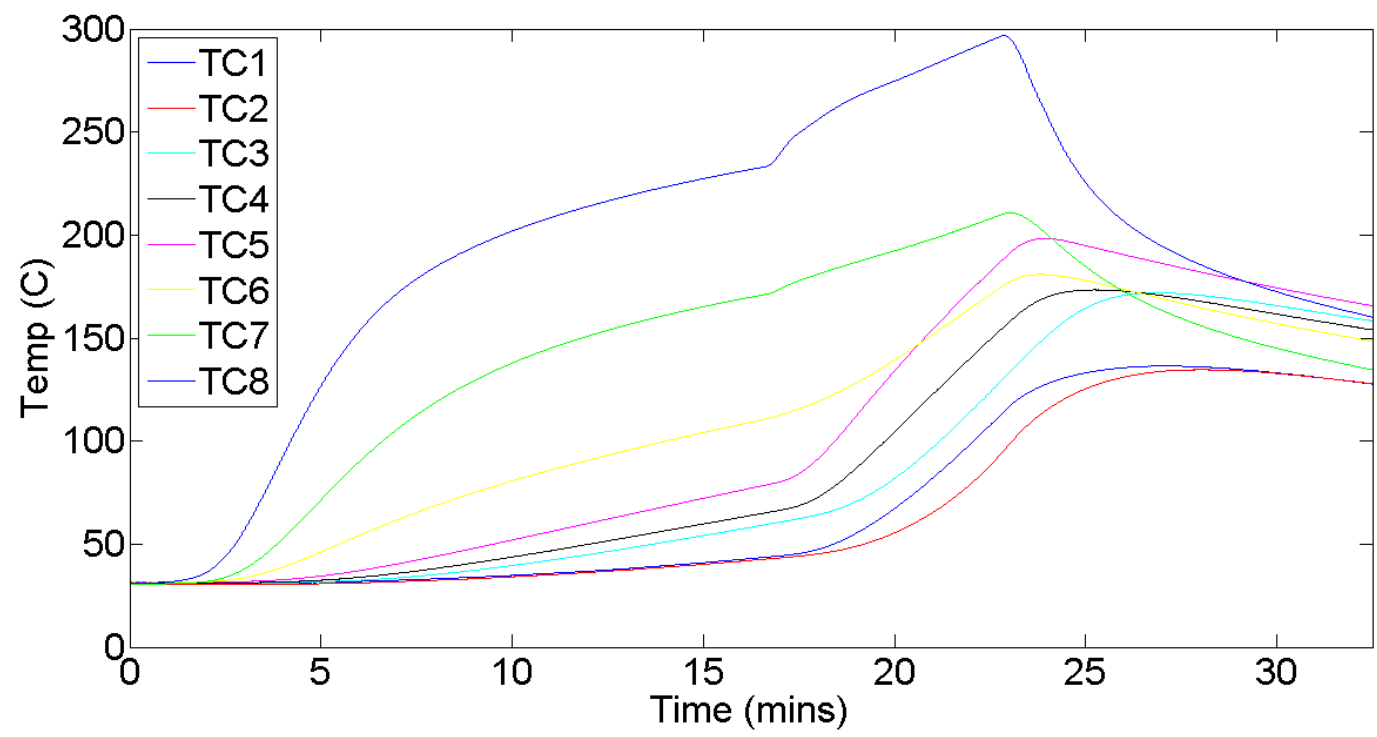

Figure C.8: Test 8.

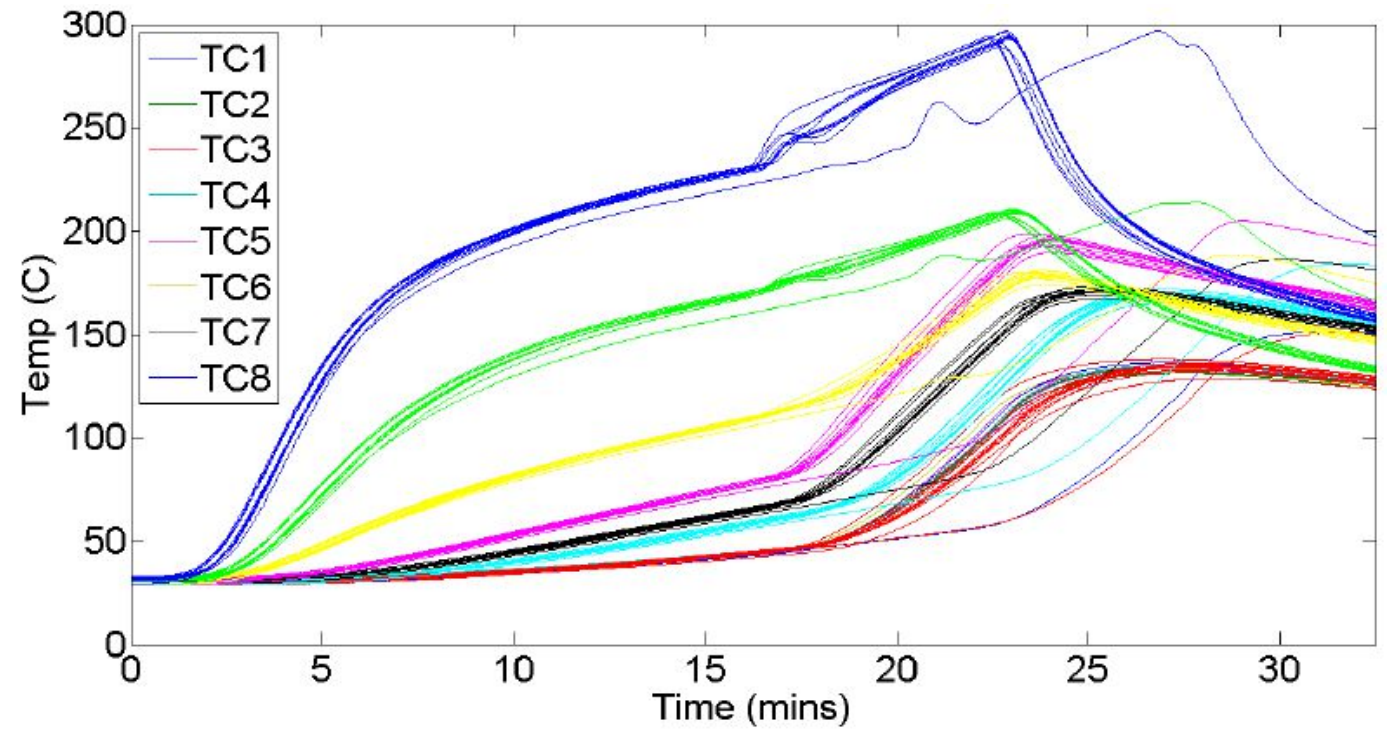

Figure C.9: All of the data that was collected during the 11 tests. The data from the first test is shifted to the right of all subsequent tests because it took several minutes longer for the keeper to strike. Therefore, data from this test was considered to be an out-lier and thrown out of final calculations. 


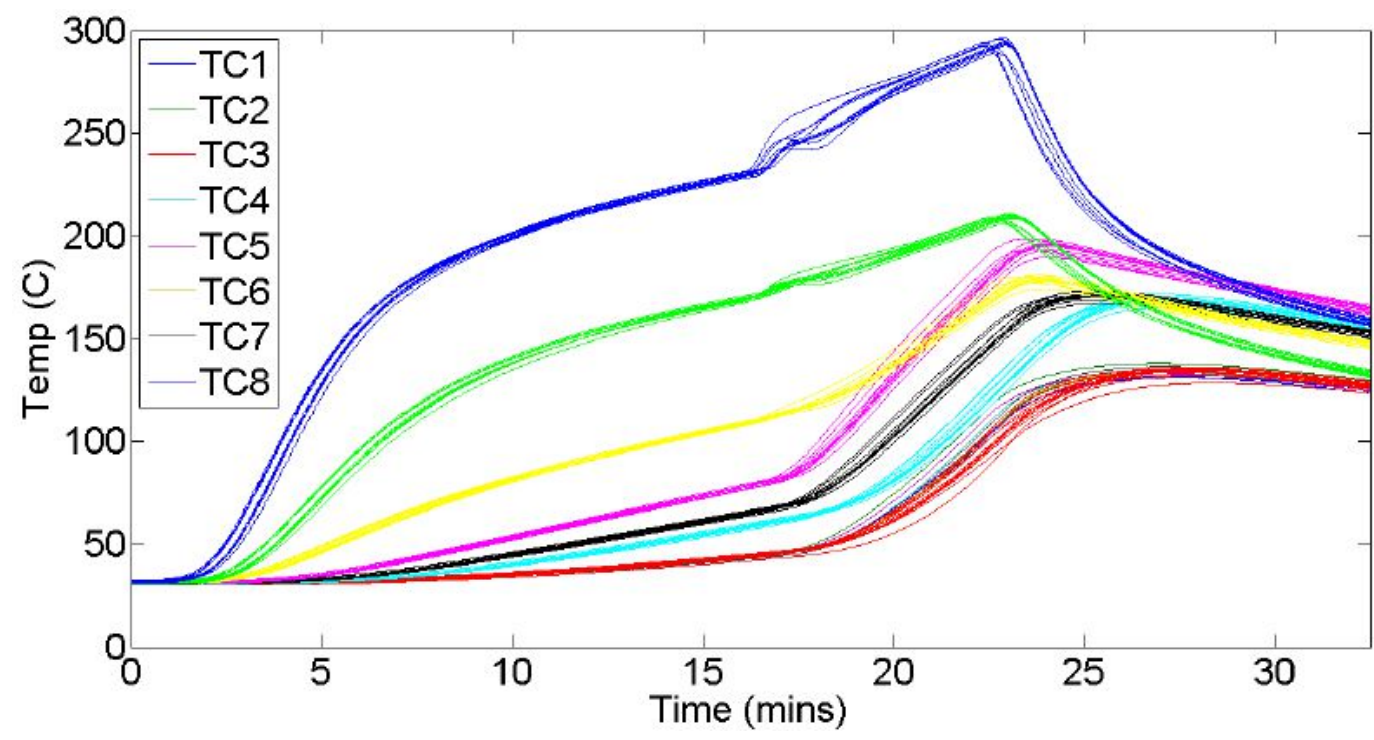

Figure C.10: All test data, excluding outliers.

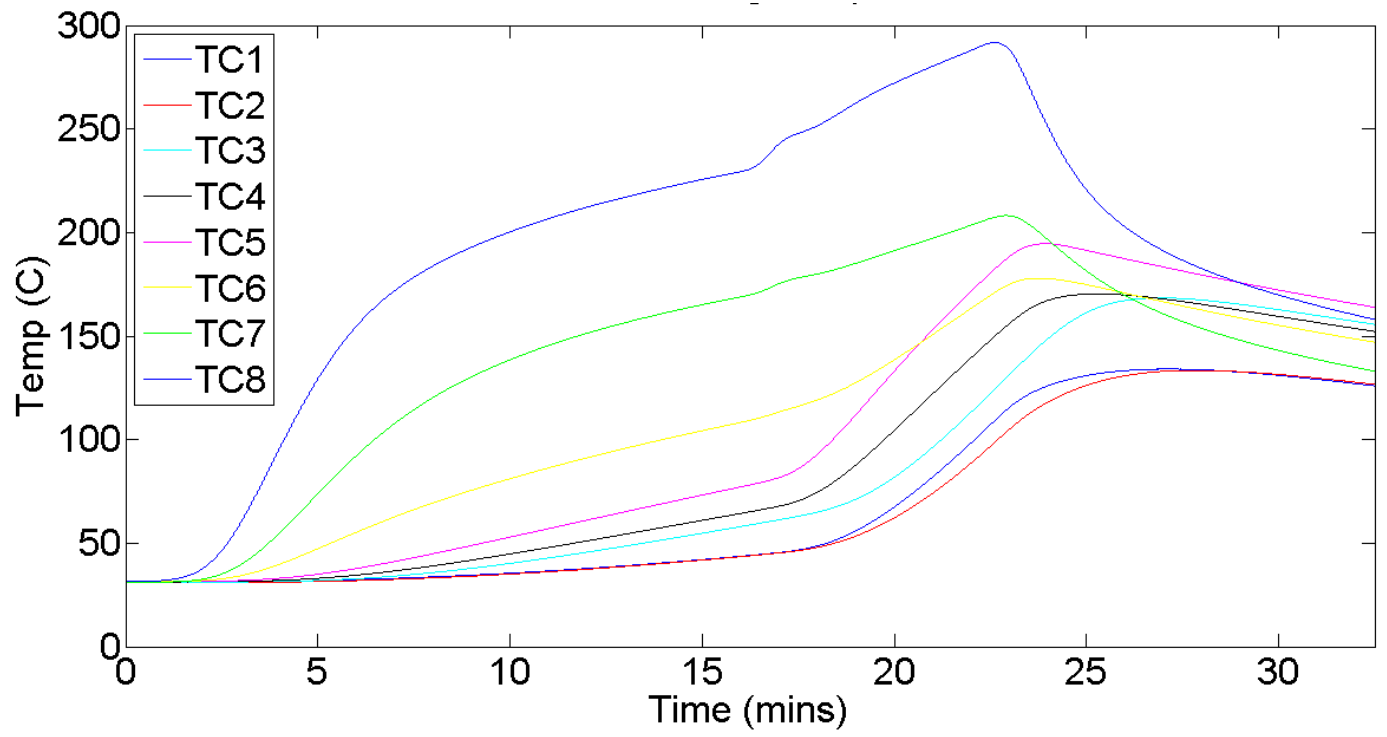

Figure C.11: The average of the 10 tests that were considered. 


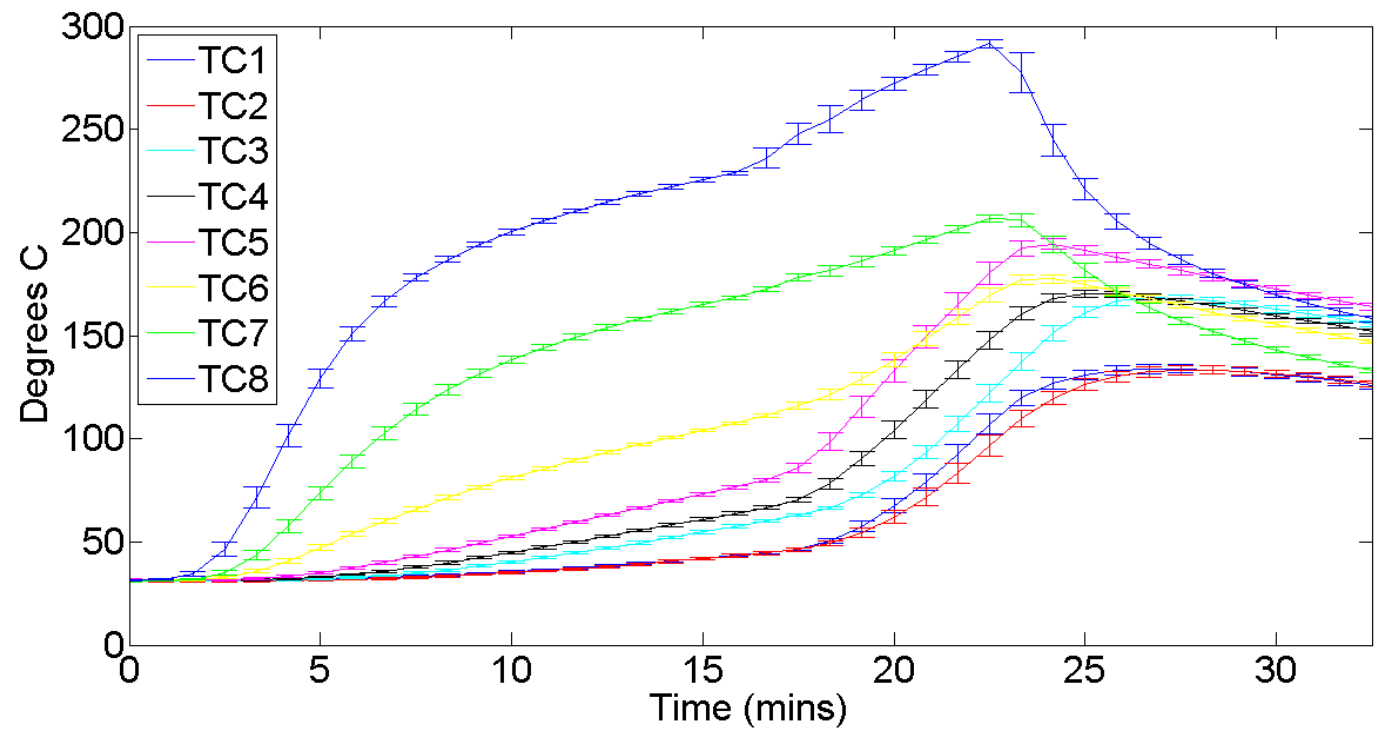

Figure C.12: The average of the 10 tests that were considered, with error bars to indicate one standard deviation from the mean at a point in time.

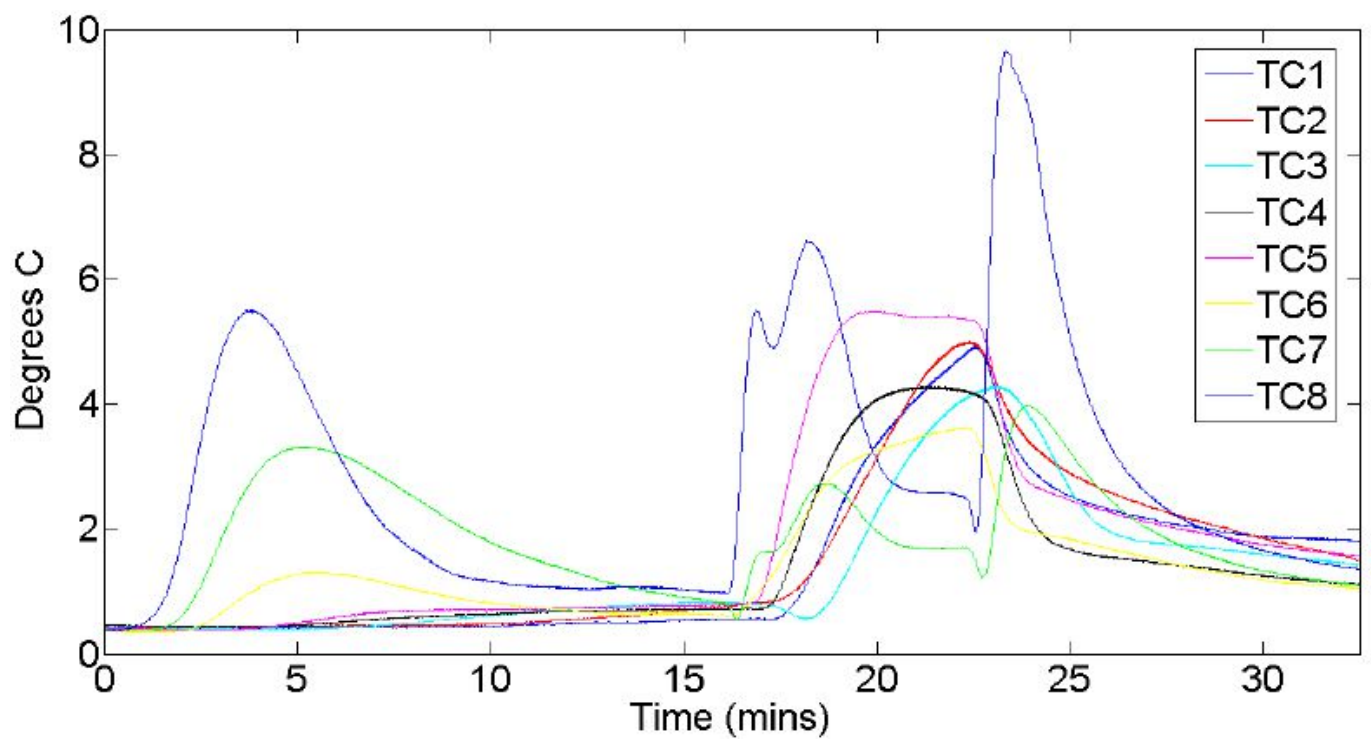

Figure C.13: One standard deviation from the mean vs time. 


\section{Procedures}

\section{D.1 High Vacuum Operation Procedure}

\section{D.1.1 Pre-Pumping Procedures and Safety Checks}

1. Ensure that all vacuum control panel toggles are switched to the off position.

2. Make sure all service panels, especially the relay cover, are closed and secure.

3. Flip the 120 VAC breaker to the "on" position.

4. Open the ball valve to the pressurized air line.

5. Check the pressurized air regulator and ensure that it reads between 70-75 psi.

6. Turn on the Main Power on the vacuum control panel.

7. Turn on the Cryogenic Temperature Indicator.

8. Turn on the Granville-Phillips 375 Vacuum Gauge Controller.

- Convectron gauge 2 (CG2) indicates cryopump pressure in torr.

- Convectron gauge 3 (CG3) indicates chamber pressure in torr. 


\section{D.1.2 Cryopump Roughing Procedure}

1. Turn on the "Mechanical Pump" on the vacuum control panel.

2. Check the sight glass on the back of the Welch 1397/1374 roughing pump to ensure the oil level is acceptable. If it is not see manual for procedures.

3. Turn on the "Rough Interlock Valve" on the vacuum control panel.

4. Turn on the "Cryo Rough Valve" on the vacuum control panel.

5. Monitor the 375 Vacuum Gauge Controller and ensure that cryopump pressure is falling.

6. Once the cryo pressure reads below $150 e^{-3}$ Torr (150 milli Torr) proceed to Cryopump Compressor Procedure.

Note: It is preferential for the cryopump pressure to be as low as possible upon cryopump compressor startup. Beginning Cryopump Compressor Procedure at 50 milliTorr has shown marked improvement in pumping speeds. However, if roughing pump performance has degraded, compressor procedures can commence at 150 milliTorr.

7. If cryopump pressure never reaches 150 milliTorr or the pressure is decreasing at an unacceptable rate, proceed to Martin-Victor Cycling Procedure.

\section{D.1.3 Martin-Victor Cycling Procedure}

Note: This procedure is only to be used when the roughing pressure ceases to decrease to the required vacuum or decreases at an extremely slow rate when 
using the roughing pump.

1. Close the roughing valve.

2. Immediately turn off the roughing pump.

3. Wait 30 seconds.

4. Turn on the roughing pump.

5. Wait 10 seconds.

6. Open the roughing valve. The pressure will initially rise, but then lower below the previous threshold. Repeat these procedures as necessary to achieve the required vacuum.

\section{D.1.4 Cryo Pump Compressor Procedure}

1. Make sure that the compressor is plugged into the $208 \mathrm{~V}, 3$ phase outlet on the vacuum chamber.

2. Verify that the helium pressure on the Compressor is 250 psi.

Note: If the helium pressure is incorrect do not operate the compressor. If this is the case please refer to the Helium Addition Procedures for the specific compressor.

3. Verify that the cover limit switch is depressed. The compressor will shut off if this is not actuated. 
Note: If the compressor ceases to operate at any point this is the first area the operator should check.

4. Make sure that the "Cold Head" and Compressor power switches on the front ofthe compressor are on.

5. Turn on the "Compressor" switch on the vacuum control panel.

6. Verify that the running helium pressure is 285 psi. The needle will oscillate by 5 psi. This is normal and not a cause for concern.

7. Observe the cold head motor to ensure that it rotates clockwise. Listen for any signs of seizing or grinding.

8. Monitor the Cryo pump temperature indicator and verify that the cryo temperature begins to fall.

Note: The temperature will begin to fall slowly until it reaches a base operating temperature between $1020 \mathrm{~K}$. This process typically takes between $1.5-2.5$ hours. Once the temperature drops below $150 \mathrm{~K}$, close the cryo roughing valve.

9. Turn off the mechanical pump

Note: The pressure in the cryo pump must remain below 150 milliTorr throughout cryo pump operation. If the pressure rises, the compressor and cryo pump should be immediately shut off and allowed to return to ambient temperatures before troubleshooting can begin. 
10. Monitor the compressor throughout the procedure to ensure that overheating doesnot occur.

\section{D.1.5 Experimental Loading Procedure}

1. Turn on the "Vent Switch" on the vacuum control panel.

2. Monitor the Granville-Phillips 375 Vacuum Gauge Controller to ensure that the chamber pressure rises to levels at or above atmospheric pressure (760 torr).

3. Turn on chamber vent valve.

4. Raise the bell jar using the hoist controls

Note: Use the "slow" buttons when raising or lowering the bell jar within four inches of the chamber. The fast buttons can be used to raise or lower the bell jar to loading heights.

5. Install the experimental setup according to the goals of your given test.

6. Ensure that all materials being placed in the chamber have acceptable outgassing levels to avoid component contamination

7. Check the bell jar gasket to ensure a complete coating of high vacuum grease.

8. Ensure all components of the experiment will not interfere with the vacuum chamber seal or the gate/poppet valve operation

9. Lower the bell jar. 
10. Check the perimeter of the bell jar to ensure a uniform seal.

\section{D.1.6 Chamber Roughing Procedure}

1. Turn on the "Mechanical Pump" on the vacuum control panel.

2. Turn on the "Chamber Roughing" on the vacuum control panel.

3. Monitor the chamber pressure on the Granville-Phillips 375 Vacuum Gauge Controller.

4. When the chamber pressure reaches 50 milliTorr, shut off the "Chamber Roughing" on the vacuum control panel.

\section{D.1.7 Cryotorr Pumping Procedure}

Note: These procedures can commence once the cryopump temperature is below $20 \mathrm{~K}$ and the chamber pressure at 50 milliTorr. However, the cryopump should be allowed to drop to its lowest base temperature for optimal high vacuum performance.

1. Turn off the Chamber Rough switch on the vacuum control panel.

2. Turn off the Mechanical Pump switch on the vacuum control panel.

3. Turn on the Pressure Interlock on the vacuum control panel.

4. Open the high vacuum valve by switching on the Gate Valve switch on the vacuumcontrol panel. 
5. Within moments the chamber pressure on the Granville-Phillips 375 Vacuum Gauge Controller will read 0 millitTorr as the pressure quickly drops. Once this occurs turn on the Ionization Gauge (IG) on the Granville-Phillips 307 Vacuum Gauge Controller.

Note: Turning the ion gauge before the chamber pressure reaches 0 milliTorr will result in permanent ion gauge damage.

6. Experimentation can commence once the pressure reaches the desired vacuum pressure pending that it is within the capabilities of the vacuum chamber.

\section{D.1.8 Experiment Removal Procedure}

1. Turn off the Granville-Phillips 307 Vacuum Gauge Controller.

2. Switch off the Gate Valve to close the high vacuum valve.

3. Switch off Compressor to turn off the cryo pump.

4. Turn off the Pressure Interlock on the vacuum control panel.

5. Turn on the Vent Valve to vent to chamber.

6. Once the chamber pressure reaches atmospheric levels, raise the bell jar.

7. Remove experimental equipment

\section{D.1.9 Shut Down Procedure}

1. Lower the bell jar. 
2. Follow the chamber roughing procedures and pump the chamber down to below 100 milliTorr because the vacuum chamber should be stored under vacuum

3. Close the chamber roughing valve

4. Shut off the mechanical pump

5. Verify all vacuum chamber components (pumps and valves) are shut off on the vacuum control panel.

6. Turn off the Granville-Phillips 375 Vacuum Gauge Controller.

7. Turn off the air pressure main by closing the ball valve.

8. Turn off the 120 VAC power breaker.

\section{D.1.10 Regeneration Procedure}

1. Follow Cryotorr pumping and chamber rouging procedures.

2. Pressure override on.

3. Open gate valve.

4. Compressor off.

5. Turn on the roughing pump.

6. Open chamber roughing valve.

7. Leave the roughing pump on while the cryo heats up to ambient temperature. This should take 3-5 hours.

8. Close Gate valve. 
9. Switch off pressure override.

10. Turn off the roughing pump.

\section{D.1.11 Tips and Tricks}

- The chamber pressure in MAX is capable of reaching as low as 8 mili Torr using only the roughing pump. It can reach this pressure when the roughing pump has been operating for 1-2 hours. To ensure the lowest possible base pressure, allow the chamber to rough out to at least 10 miliTorr before opening the gate valve to the Cryo chamber.

- When utilizing the Cryo, the base pressure in MAX has gotten as low as $5.4 e^{-7}$ Torr. It is unlikely that the chamber will reach a lower base pressure without significant (and costly) modifications.

- If the desired base pressure cannot be reached, run the chamber through a regeneration cycle. This is time consuming so it's best to do it overnight.

- Allowing the ion gauge to DGAS for 5-10 minutes before each test will clean off all of the debris that it has collected and allow the gauge to collect a more accurate reading.

- If the desired base pressure still cannot be reached, one of the quickest remedies is to remove the bell jar as well as all of the smaller caps and feedthroughs. Remove the O-rings and clean them with water (do not clean the O-rings with acetone). Wipe down all feedthrough caps and interfaces with acetone. Clean the vacuum grease off the bell jar's O-rings and replace it with a new coat of grease. Apply a fresh coat of vacuum grease to the O-rings. Put the O-rings back on the caps and feedthroughs. Replace the 
feedthroughs and tighten them as much as possible. This will close up most of the leaks faster than attempting to find them with the leak detector.

Note: Using a helium leak detector may seem like the easier option, but it can be far more time consuming if the leak is not discovered right away.

- If the compressor turns on and only runs for several seconds before switching off, check the window on of the Cryo chamber's cold head. If the dial makes less than one rotation clockwise or counter clockwise while making a grinding noise, then the power to or within the compressor may be out of phase. Try plugging the compressor into another outlet. If the compressor and cold head function properly while the compressor is plugged into another outlet, then there may be a short in the circuit for the 3-phase power outlet on the vacuum chamber. If the problem persists when the compressor is plugged into another outlet, then the problem is internal to the compressor; in this case, refer to the compressor's operators manual.

- If a fastener or other small object falls into the bottom of the chamber, remove it BEFORE pumping down the chamber and opening the gate valve. A small nut or bolt could jam the cold head if it is allowed to fall into the cryo pump.

\section{D.2 Hollow Cathode Operating Procedures}

The following section describes the procedures for operating the Hollow Cathode without MiXI in Cal Poly's MAX Vacuum Chamber. A list of required electrical equipment is provided in Table D.1, while a list of equipment for the propellant system is provided in Table D.2. Items that are also needed but not listed are: 
the Minimum Atmospheric eXperimentation (MAX) vacuum chamber, the anode, Kimtech 'Delicate Task Wipes', a supply of acetone, powder-free rubber gloves, the vacuum chamber test stand, and (obviously) the hollow cathode.

Table D.1: Electrical Equipment Required for Hollow- Cathode

\begin{tabular}{|l|c|c|l|l|}
\hline Item & Model & Quantity & Description \\
\hline Keeper Power Supply & Agilent N5771A & 1 & $300 \mathrm{~V} / 5 \mathrm{~A}$ \\
\hline Heater Power Supply & HP 6038A & 1 & $60 \mathrm{~V} / 10 \mathrm{~A}$ & \\
\hline Discharge Power Supply & Instek GPS2303 & 1 & $30 \mathrm{~V} / 6 \mathrm{~A}$ & \\
\hline Igniter Power Supply & Glasman FC1P120 & 1 & $1000 \mathrm{~V} / .123 \mathrm{~A}$ \\
\hline Wires w/ Ring Termials & - & 9 & 4 red and 3 black \\
\hline Banana to Ring Cable & - & 3 & 2 red and 1 black \\
\hline Alligator Clip & - & 1 & - & \\
\hline BNC to Ring Terminal & (for Glasman) & 1 & - & \\
\hline High Volt Diodes (1kV) & - & $\geq 2$ & - & \\
\hline High Amp Diodes (3A) & - & $\geq 2$ & - & \\
\hline Thermocouple (TC) & K type & $\geq 1$ & $\begin{array}{l}\text { To ensure no } \\
\text { overheating }\end{array}$ \\
\hline TC Reader & Omega HH85 & $\geq 1$ & $\begin{array}{l}\text { To ensure no } \\
\text { overheating }\end{array}$ \\
\hline
\end{tabular}

Table D.2: Hollow Cathode Propellant System

\begin{tabular}{|c|c|l|}
\hline Item & Quantity & Description \\
\hline Propellant Supply & 1 & $\begin{array}{l}99.999 \% \text { pure Xenon or Ar- } \\
\text { gon }\end{array}$ \\
\hline Propellant Isolator & 1 & $\begin{array}{l}\text { Electrically isolates propel- } \\
\text { lant lines from the cathode }\end{array}$ \\
\hline Diaphragm Valve (optional) & 1 & Swagelok SS 4H \\
\hline Needle Valve & 1 & VACOA MV-25 \\
\hline Flow Meter & 1 & Omega FMA-A2300 \\
\hline Regulator & 1 & Smith Stainless Steel \\
\hline Pipe Sections & - & $\begin{array}{l}\text { 1/4" aluminum pipe with } \\
\text { Swagelok connectors }\end{array}$ \\
\hline
\end{tabular}




\section{D.2.1 Pre-Setup Procedure}

1. Locate the hollow cathode and all supporting equipment required for operation.

2. Using acetone and 'Delicate Task Wipes', clean the interior of the vacuum chamber, including the bell jar.

3. While wearing powder-free rubber gloves, use acetone to clean the hollow cathode, anode, propellant isolator, and any other metallic objects that will be placed in the vacuum chamber. Allow 4 to 6 hours to dry. (In order to reduce the risk of contaminating the cathode, it is important to use gloves when handling this equipment after it has been cleaned.)

4. Optional: Run the vacuum chamber through a regeneration cycle (see Vacuum Operation Procedures).

\section{D.2.2 Hollow Cathode Loading Procedure}

NOTE: Read Experimental Loading Procedure in the vacuum chamber procedures prior to loading the cathode into the chamber.

1. Open the vacuum chamber and carefully remove the jar. Place the jar on a clean surface while the experiment is being installed.

2. While the chamber is empty, identify which of the electrical feedthroughs and thermocouples will be used. For this test, you will need at least one thermocouple.

3. Install the chamber test stand in the vacuum chamber. 
4. Place the propellant isolator on the test stand and connect to the propellantline feed-through using the appropriate section of pipe.

5. Place the hollow cathode on the test stand and attach the propellant line to the propellant isolator.

6. Place the anode outside the exit of the hollow cathode. Ensure that the cathode and anode are not in contact.

\section{D.2.3 Electrical Setup Procedure}

This section covers the electrical setup of the hollow cathode prior to testing. Set up the wiring according to fig D.1. Use the steps below for guidance. Be sure to shut down all of the power supplies when they're not in use. To avoid electric shock, be sure to insulate all exposed terminals and diodes.

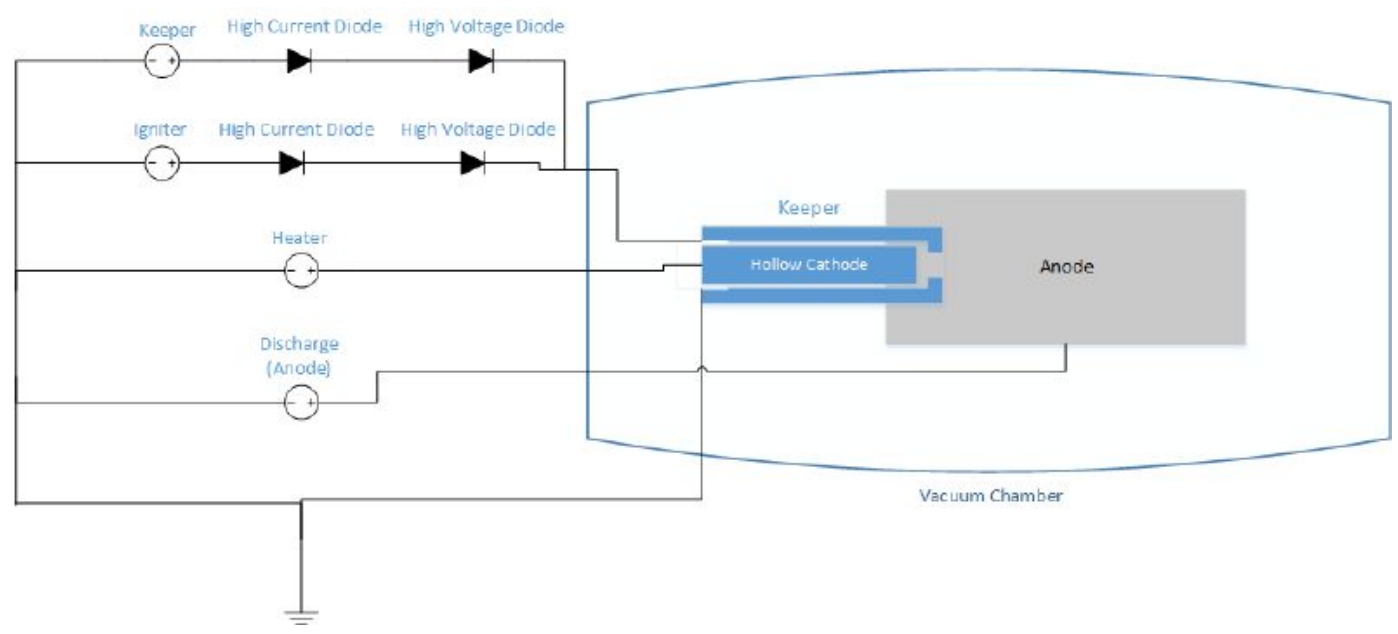

Figure D.1: Hollow Cathode Circuit Diagram.

1. Ensure that all of the power supplies are turned off and unplugged prior to setup. 
2. Attach the red ring terminal cables to the Keeper and Heater attachment points on the back of the cathode. Refer to fig D.2 for the attachment points. Attach the opposite end of these cables to their respective feedthroughs on top of the chamber.

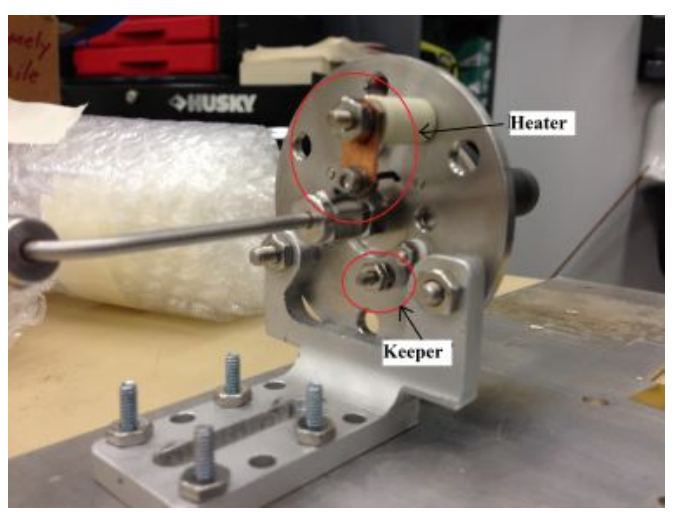

\section{Figure D.2: Heater and Keeper attachment points.}

3. Attach a black ring terminal cable to the chassis of the cathode. This will provide the attachment point for all grounds. Attach the opposite end of this cable to one of the feedthroughs at the top of the chamber.

4. Attach and alligator clip to the red banana to ring cable. Clamp the alligator clip to the anode and secure it using kapton tape. Attach the opposite end of this cable to one of the remaining feedthroghs at the top of the chamber.

5. Use the Glasman's BNC to ring terminal to connect the igniter supply to the keeper's positive (red) electrical feedthrough. Be sure to have at least one high voltage and one high current diode in series between the Glasman power supply and the keeper feedthrough.

6. Using a cable with ring terminals, connect the positive terminal of the 
keeper power supply to the keeper's positive (red) electrical feedthrough (the same feedthrough that the igniter supply is connected to). Be sure to have at least one high voltage and one high current diode in series between the keeper power supply and the keeper feedthrough.

Note: The diodes are very important and are necessary to prevent current form flowing to the positive terminal of the keeper and igniter power supplies. The high voltage diode should be placed downstream of the high current diode. Once the cathode has ignited, current can exceed the limits of the high voltage diodes and cause them to fail. It is necessary to have a redundant high current diodes to prevent this current from reaching one of the power supplies.

7. Using kapton tape, attach a thermocouple to the chassis of the cathode.

8. Carefully replace the bell jar. DO NOT allow the bell jar to come into contact with any of the test equipment. If necessary, apply high-vacuum grease to the jar's O-rings prior to this step.

9. Outside the chamber, use the red ring terminal cables to attach the positive terminals of the heater and anode power supplies to their respective feedthroughs. Ensure that the power supplies are unplugged to avoid electric shock.

10. Use three black ring terminal cables to connect the negative terminals of the heater, keeper, and anode power supplies to the ground feedthrough.

11. Perform connectivity test. Use a multimeter in connectivity mode to check the connection between the keeper terminal, heater terminal, and the cath- 
ode chassis. The multimeter should indicate connectivity between the keeper terminal and keeper electrode and isolation from the cathode chassis and the heater terminal. The multimeter should indicate that the heater electrode IS electrically connected to the cathode's chassis and the ground wire. The anode should be isolated from everything except the positive node of the anode power supply.

12. Use zip-ties or kapton tape to secure any loose ends of cables and/or thermocouples. If cables or wires are dangling in the bottom half of the chamber, they can fall into the gap between the gate valve and the cryo chamber once the gate valve is open. This makes completely closing the gate vale impossible.

Note: Should a loose end of a wire prevent the gate valve from closing all the way, DO NOT attempt to re pressurize and open the vacuum chamber. Turn off the compressor and wait (3-4 hours) until the cryo chamber temperature has reached room temperature. Introducing air into the cryo chamber while it is cold can damage the cryo.

13. Once the connectivity check has been performed and loose ends of wires are secured, lower the hoist to close the vacuum chamber and proceed to the Propellant Line Setup Procedure.

\section{D.2.4 Propellant System Setup Procedure}

This section will detail the setup of the propellant lines outside of the vacuum chamber. Setup the propellant system according to the schematic in fig D.3 


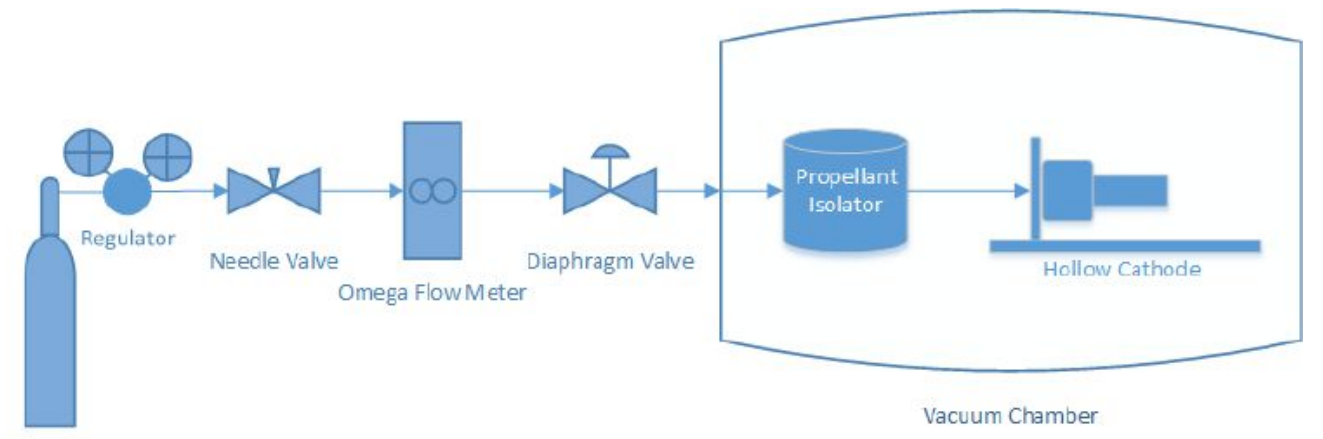

Xenon Propellant Tank

Figure D.3: Hollow Cathode Propellant System Layout.

1. Using 1/4" pipe sections with Swagelok connectors, connect the Smith regulator, needle valve, flow meter, and diaphragm valve according to fig D.4

Note: Every piece of hardware in this plumbing system will introduce a small leak into the vacuum chamber. The fewer components in the system, the lower the base pressure in the chamber. At a minimum, the propellant feed line requires the needle valve, the flow meter, and the regulator. While a diaphragm valve is desirable, the system has been successfully operated without the use of the diaphragm valve. It is also recommended that a small amount of Teflon tape and high vacuum grease be applied to the threads of the connectors.

2. Using the DC adapter, plug the flow meter into an outlet. The display should read "00.00".

3. Connect the downstream section of the diaphragm valve (or flow meter, if the diaphragm valve is not used) to the propellant line feedthrough on the chamber. 
4. Double check that all connectors have been securely fastened before proceeding.

5. Proceed to vacuum chamber procedures for instructions on pumping down the chamber.

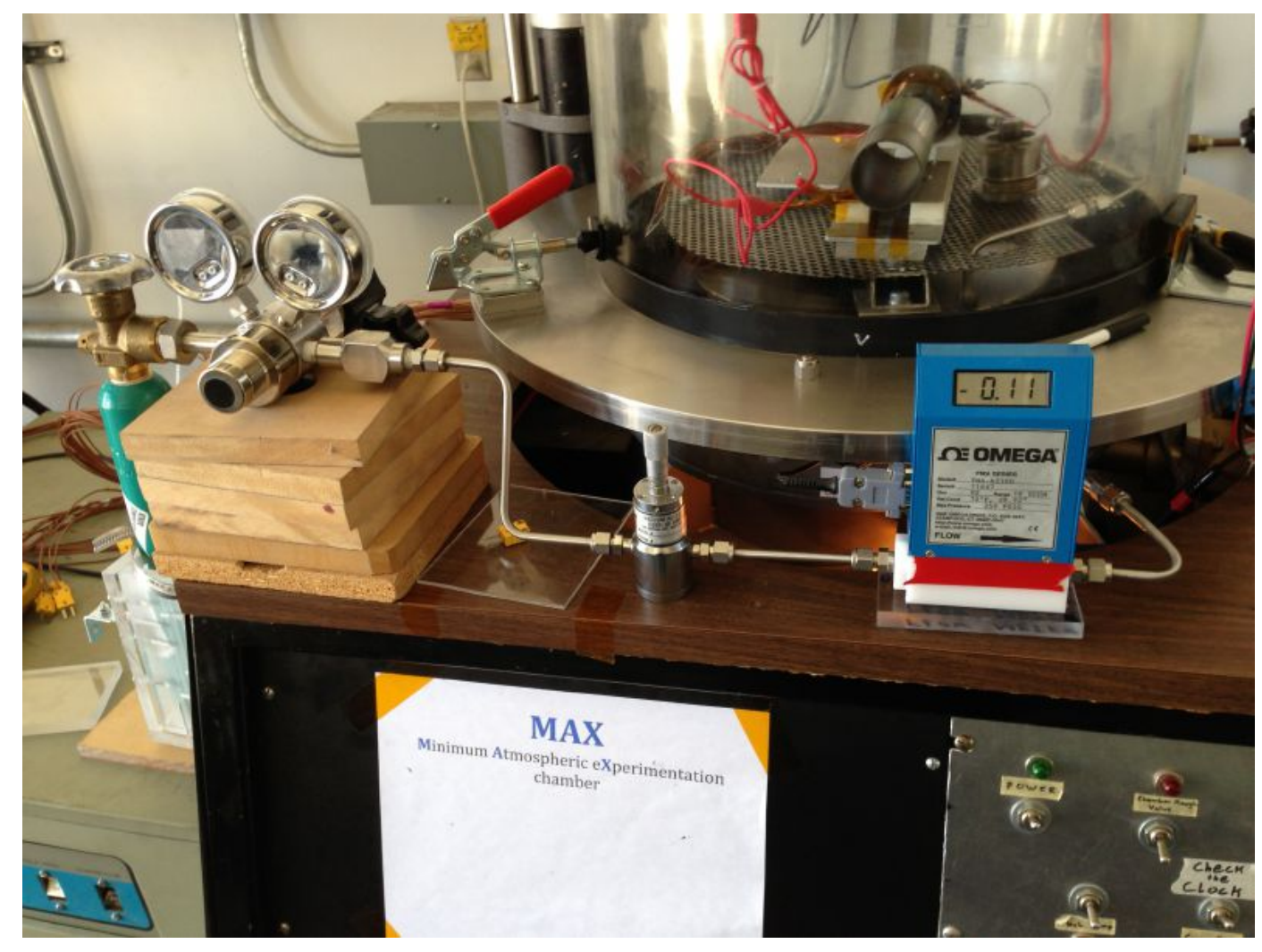

Figure D.4: MiXI/Hollow Cathode Propellant System. Notice that there is no diaphragm valve between the flow meter and the propellant feedthrough into the chamber.

\section{D.2.5 Hollow Cathode Operation Procedure}

1. (This is the most important step of the entire procedure.) While the chamber is pumping down, open all the valves in the propellant feed line up to (but not including) the propellant tank. Ensure that the diaphragm 
valve (if necessary), the needle valve, and the valve on the regulator are completely open. Turn the big knob on the Smith regulator in the direction of the Increase arrow to open the regulator. The downstream gague on the regulator should drop to the lowest reading (-30 psi).

Note: It is extremely important that there is absolutely NO AIR in the propellant feed line between the xenon tank and the cathode. If air is present in the system when the cathode is hot, the cathode insert will be poisoned.

2. Once the proper base pressure ( $\leq 5.0 e^{-6}$ Torr) has been reached, turn on the heater power supply. Set it to current controlled, 6A. Let the cathode heat up for about 15-20 minutes. Do not exceed 6.05A of current.

3. Open the propellant tank. The regulator should jump from -30 psi to $20-25$ psi.

4. Close the needle valve until the flow meter reads at least 3.5 SCCM.

Note: This flow rate includes the nitrogen-to-xenon conversion factor. The flow meter is calibrated for nitrogen so a conversion factor of 1.44 must be applied in order to determine the actual xenon flow rate.

5. Turn on the keeper power supply. It should automatically be set to voltage control. Increase the voltage until the keeper strikes. (You will know when this happens when the current indicated by the power supply abruptly spikes and the power supply switches to current control. Also, you will notice a purple glow emanating from the cathode.) Striking the cathode can theoretically occur around $120 \mathrm{~V}$, but in practice, the keeper may require 
as much as $1 \mathrm{kV}$ to strike. If the keeper does not strike at $120 \mathrm{~V}$, continue to increase the keeper voltage until it strikes or the maximum voltage of the power supply has been reached (300V for the Agilent or Sorinson supplies in lab). If the keeper strikes, you will not need the Glasman power supply and you may skip to step 8 .

Note: If the keeper supply is consistently drawing current before the voltage reaches about $50 \mathrm{~V}$, there may be a short between the keeper and the heater. Should this happen, turn everything off, wait for the cathode to cool, and perform a connectivity check on the cathode.

6. If the maximum voltage on the keeper's power supply has been reached, and the cathode has yet to ignite, then you will need to use the igniter supply (Glasman high voltage power supply). Ensure that the current and voltage knobs on the Glasman are set to zero before turning on the power supply. With the keeper power supply still on, switch on the Glasman and increase the current to $.125 \mathrm{~A}$ (the maximum for this supply). Slowly increase the voltage on the Glasman until the keeper strikes. If the Glasman voltage is increased to $1 \mathrm{kV}$ without ignition, turn off the keeper and igniter supplies and return to step 5 .

7. Once the cathode has ignited, adjust the current on the keeper supply to the desired operational range (1-3A).

Note: The keeper power supply my oscillate between current control and voltage control as the cathode sputters and attempts to ignite. When this is happening, increase the current on the keeper supply until the current 
becomes steady (usually between 1-3 Amperes). Do not adjust or shut down the Glasman power supply until the cathode current is running steadily.

8. Turn off the igniter (Glasman) power supply.

9. Turn off the heater power supply.

10. Turn on the discharge (anode) power supply. Adjust the current and voltage until the desired discharge current has been reached.

11. Slowly close the needle valve until the desired propellant flow rate is reached.

12. Allow the cathode to operate steadily as long as desired (no more than one hour).

13. Turn off the discharge and keeper power supplies, in that order.

14. Close the diaphragm and/or needle valve to stop propellant flow.

15. Allow the cathode to cool for a minimum of two hours before opening the chamber. Once all tests are complete, follow the "Experimental Removal Procedure" to remove the apparatus from the chamber.

\section{D.2.6 Troubleshooting, Tips, and Tricks}

- If the cathode is not used regularly, it will be more difficult to strike. The more time between operations, the more difficult it will be to strike the cathode.

- There really is no upper limit on the propellant flow rate during ignition. If the cathode won't strike at $3.44 \mathrm{sccm}$, increase the flow rate (open the needle valve all the way if you have to). 
- After the cathode has been ignited and the anode current has been set, the keeper and discharge supplies might begin to fluctuate rapidly as the propellant flow rate is decreased. This usually occurs when the cathode is in plume-mode and will stop when the propellant flow rate is decreased. The plasma emanating from the thruster should form a cone shape when it is not in plume mode (see fig. D.5).

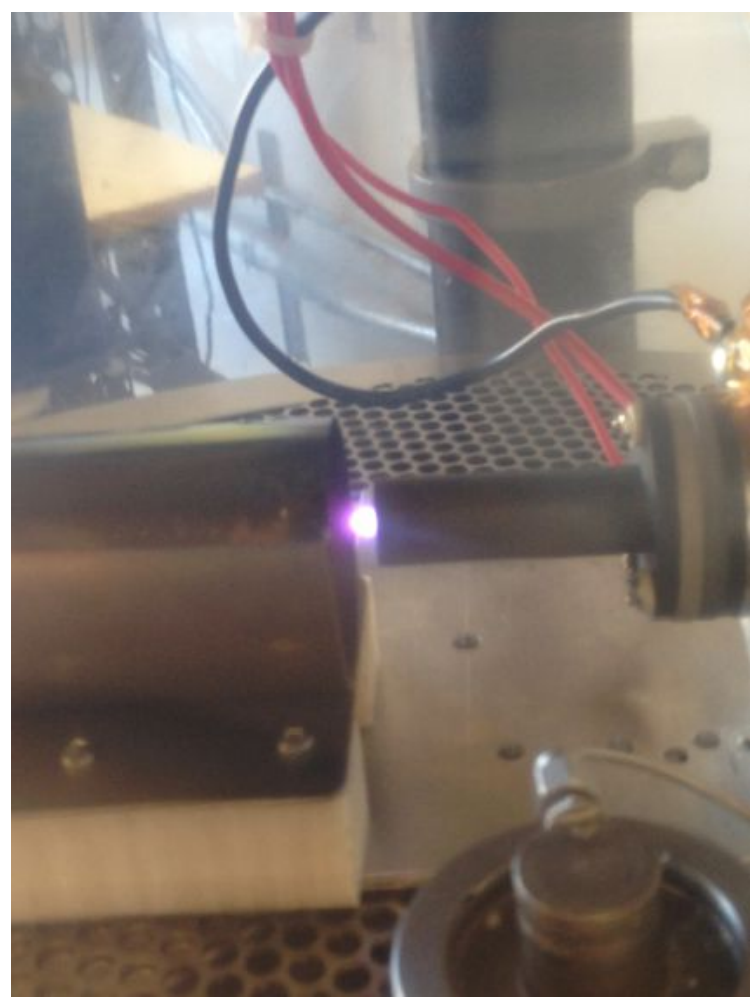

Figure D.5: Hollow cathode during nominal operation. Notice the distinct cone shape of the plasma.

- If the igniter voltage is increased to $1 \mathrm{kV}$ and the cathode still won't strike, the cathode insert may be poisoned. This can be checked by checking the current being drawn by the keeper. You'll need a multimeter that can measure current in the micro Amp range (check to make sure the fuse inside the multimeter is good). Set up the multimeter in series with the keeper and 
the igniter and keeper power supplies (in between the keeper intersection of the keeper and igniter power supplies). Then, carefully attempt to ignite the cathode. If the multimeter indicates increasing current (in the micromili amp range) as keeper voltage is increased, then the insert is okay. If the multimeter indicates zero current, then the insert is probably dead.

- If the cathode is being operated for the first time after a long hiatus, allow it to run steadily for 30 to 60 minutes. Ion bombardment will help clean the interior of the cathode and it will ignite much easier on the next attempt.

- If the inside of the bell jar forms a greasy fog while the cathode is hot, there may be too much vacuum grease on the bell jar. This will not harm the cathode, but the issue should be resolved and the chamber should be cleaned before the next test.

- If the cathode is sputtering after the discharge (anode) supply has been turned on, the discharge voltage may be too low. Try slowly adjusting the discharge voltage until the sputtering stops (avoid exceeding $5 \mathrm{~A}$ of discharge current). The sputtering may also be the result of the flow rate being too high. Double check to make sure that the flow rate is below $3 \mathrm{sccm}$.

- If the cathode is intended to be used with MiXI or another experiment, it is recommended that the user practice operating the cathode multiple times before proceeding with their experiment. Igniting the cathode is a difficult process and the user should be comfortable doing so before attempting an experiment or data collection.

- Don't ever force the plastic knob on the regulator to turn if it's stuck. You may end up stripping the knob. If this happens, you'll have to send the regulator to the manufacturer for repairs. 


\section{D.3 MiXI-CP-V3 Procedures}

This section describes the setup and operation procedures for MiXI CP-V3 in Cal Poly's Minimum Atmospheric eXperimentation chamber. A list of electrical equipment is provided in Table D.3, and a list of equipment for the propellant system is provided in Table D.3. Items that are also needed but not listed are: the Minimum Atmospheric eXperimentation (MAX) vacuum chamber, Kimtech 'Delicate Task Wipes', a supply of acetone, powder-free rubber gloves, the vacuum chamber test stand, the hollow cathode, the neutralizer CTFC, and MiXI.

Table D.3: Electrical Equipment Required for Hollow- Cathode

\begin{tabular}{|l|c|c|l|l|}
\hline Item & Model & Quantity & Description \\
\hline Keeper Supply & Agilent N5771A & 1 & $300 \mathrm{~V} / 5 \mathrm{~A}$ \\
\hline Heater Supply & HP 6038A & 1 & $60 \mathrm{~V} / 10 \mathrm{~A}$ \\
\hline Discharge Supply & Instek GPS2303 & 1 & $30 \mathrm{~V} / 6 \mathrm{~A}$ \\
\hline Igniter/Screen Supply & Glasman FC1P120 & 1 & $1000 \mathrm{~V} / .123 \mathrm{~A}$ \\
\hline Accel Supply & Sorenson XG 300 & 1 & $300 \mathrm{~V} / 5 \mathrm{~A}$ \\
\hline $\begin{array}{l}\text { CTFC Neutralizer Sup- } \\
\text { ply }\end{array}$ & HP 6263B & 1 & $20 \mathrm{~V} / 10 \mathrm{~A}$ & \\
\hline Wires w/ Ring Terminals & - & - & - \\
\hline Banana to Ring Cable & - & 3 & 2 red and 1 black \\
\hline BNC to Ring Terminal & (for Glasman) & 1 & - \\
\hline High Volt Diodes (1kV) & - & $\geq 2$ & - \\
\hline High Amp Diodes (3A) & - & $\geq 2$ & - \\
\hline Resistors & - & 2 & $\begin{array}{l}1-5000 \Omega \text { and } 1- \\
100 \Omega\end{array}$ \\
\hline Switch & - & 1 & $\begin{array}{l}\text { Igniter power to } \\
\text { screen power }\end{array}$ \\
\hline TC & K type & $\geq 1$ & $\begin{array}{l}\text { To ensure no } \\
\text { overheating }\end{array}$ \\
\hline TC Reader & & $\geq 1$ & $\begin{array}{l}\text { To ensure no } \\
\text { overheating }\end{array}$ \\
\hline
\end{tabular}


Table D.4: Hollow Cathode Propellant System

\begin{tabular}{|c|c|l|}
\hline Item & Quantity & Description \\
\hline Propellant Supply & 1 & $\begin{array}{l}99.999 \% \text { pure Xenon or Ar- } \\
\text { gon }\end{array}$ \\
\hline Propellant Isolator & 1 & $\begin{array}{l}\text { Electrically isolates propel- } \\
\text { lant lines from the cathode }\end{array}$ \\
\hline Diaphragm Valve (optional) & 1 & Swagelok SS 4H \\
\hline Needle Valve & 1 & VACOA MV-25 \\
\hline Flow Meter & 1 & Omega FMA-A2300 \\
\hline Regulator & 1 & $\begin{array}{l}\text { Smith Stainless Steel } \\
1 / 4 \text { " aluminum pipe with } \\
\text { Swagelok connectors }\end{array}$ \\
\hline Pipe Sections & - &
\end{tabular}

\section{D.3.1 Pre-Setup Procedure}

1. Locate MiXI, the hollow cathode, and all supporting equipment required for operation.

2. Using acetone and 'Delicate Task Wipes', clean the interior of the vacuum chamber, including the bell jar.

3. Carefully remove MiXI's accelerator and screen grids.

4. While wearing powder-free rubber gloves, use acetone to clean the hollow cathode, anode, propellant isolator, and any other metallic objects that will be placed in the vacuum chamber. Clean the interior of MiXI's discharge chamber and grids. Allow 4 to 6 hours to dry. (In order to reduce the risk of contaminating the cathode, it is important to use gloves when handling this equipment after it has been cleaned.)

5. Optional: Run the vacuum chamber through a regeneration cycle (see Vacuum Operation Procedures). 
6. Replace MiXI's screen and accelerator grid. Be sure to align the grid apertures as precisely as possible. Use a multimeter in connectivity mode to ensure that the screen, accel grid, and discharge chamber are isolated from one another.

\section{D.3.2 MiXI-CP-V3 Loading Procedure}

NOTE: Read Experimental Loading Procedure in the vacuum chamber procedures prior to loading MiXI into the chamber.

1. Attach MiXI to the hollow cathode's chassis using \#6 screws and 4 alumina isolators as illustrated by fig D.6. Isolate the discharge chamber from the keeper electrode using the electrode ring isolator.

Alternatively, MiXI can be secured to its own test stand if a test stand is available.

2. Open the vacuum chamber and carefully remove the jar. Place the jar on a clean surface while the experiment is being installed.

3. While the chamber is empty, identify which of the electrical feedthroughs and thermocouples will be used. For this test, you will need at least one thermocouple.

4. Install the chamber test stand in the vacuum chamber.

5. Place the propellant isolator on the test stand and connect to the propellant system feed-through using the appropriate section of pipe.

6. Place the hollow cathode/MiXI setup on the test stand and attach the propellant line to the propellant isolator. 


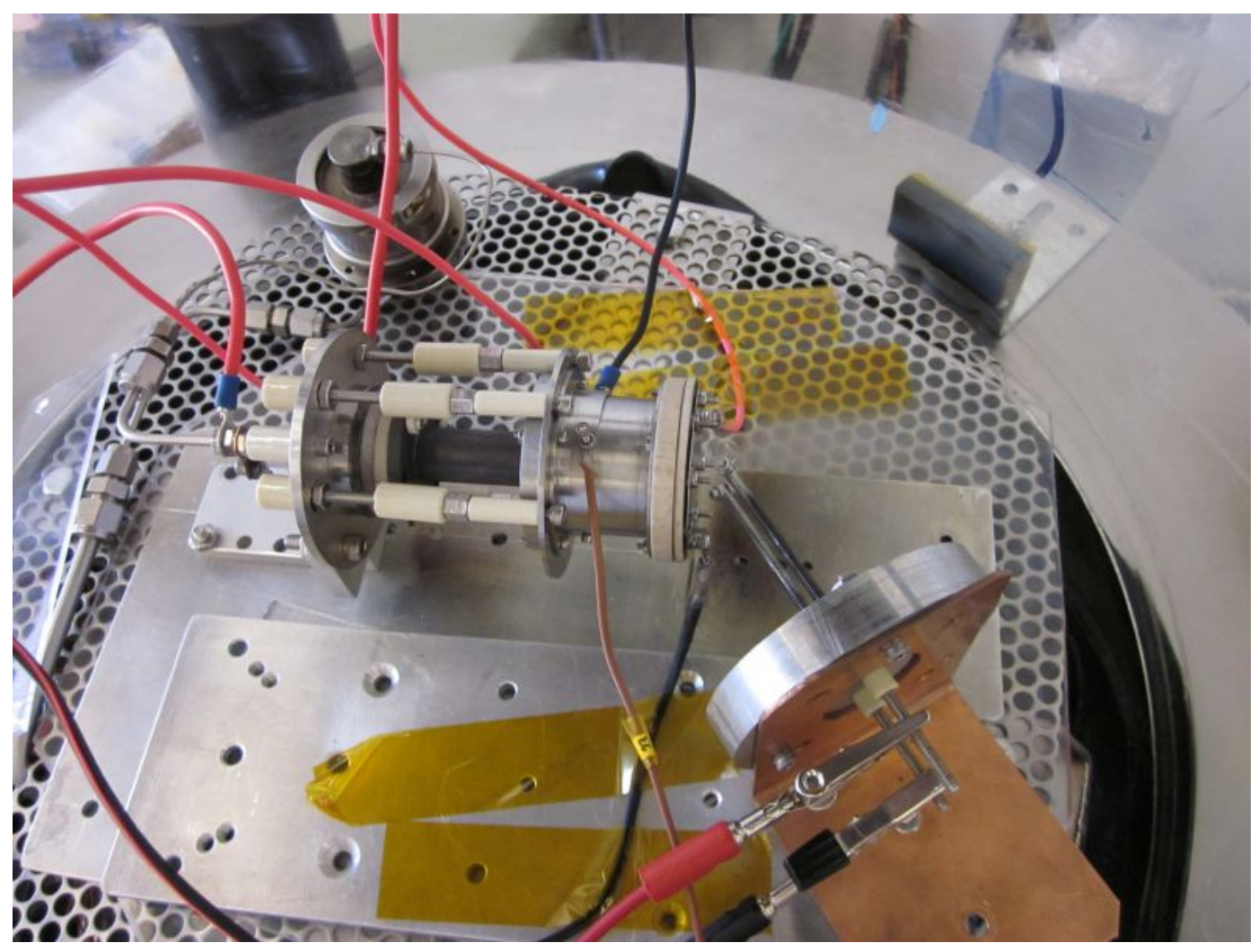

Figure D.6: MiXI test setup.[15]

7. Place the neutralizer CTFC outside the exit of MiXI.

\section{D.3.3 Electrical System Setup Procedure}

This section covers the electrical setup of MiXI prior to testing. Set up the wiring according to fig D.7. Use the steps below for guidance. Be sure to shut down all of the power supplies when they're not in use. To avoid electric shock, be sure to insulate all exposed terminals and diodes.

1. Ensure that all of the power supplies are turned off and unplugged prior to setup.

2. Set up the keeper and heater power supplies according to the Hollow Cath- 


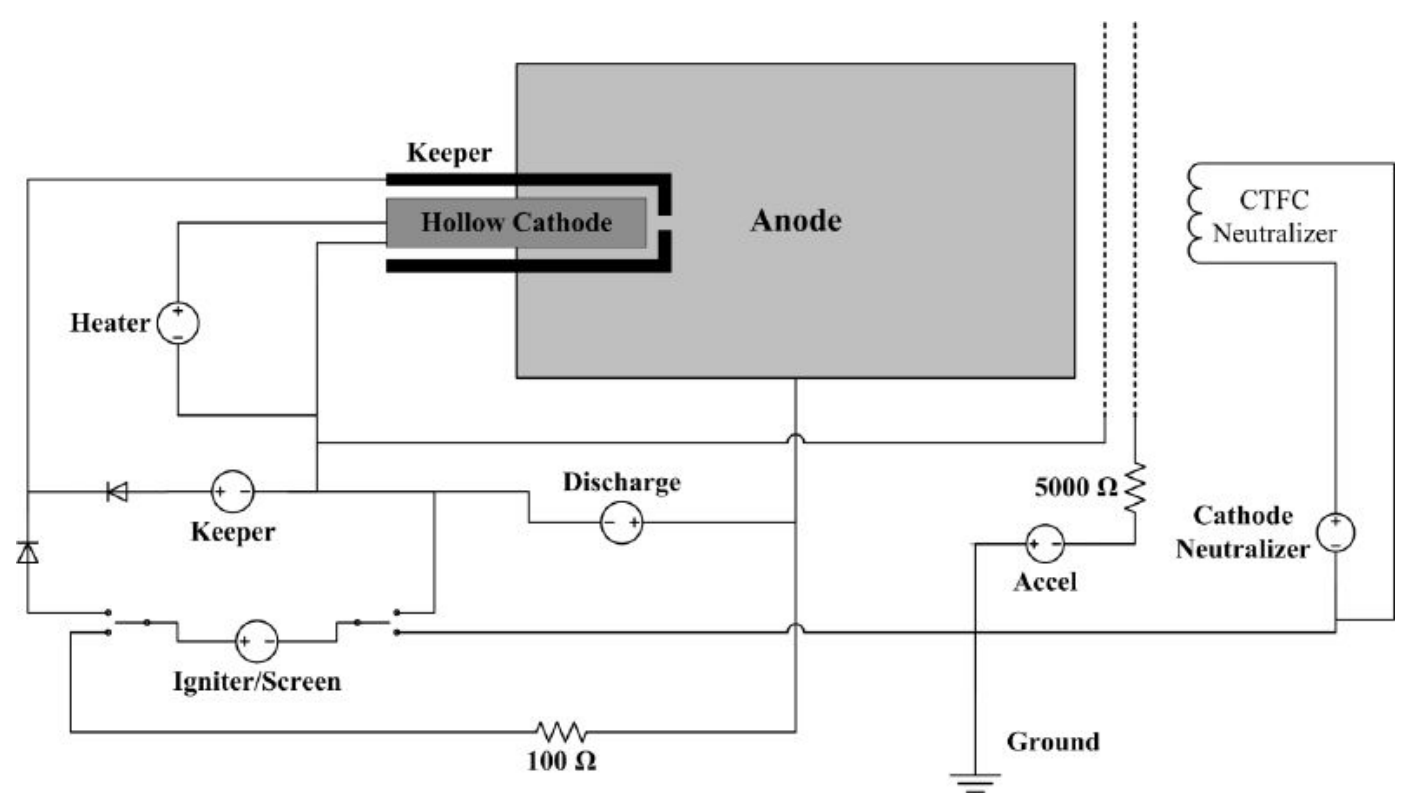

Figure D.7: MiXI Circuit Diagram.[15]

ode's Electrical Setup Procedure.

3. Connect the negative terminal of the accel power supply to the accel grid. connect the positive terminal to ground. Ensure there is a $5000 \mathrm{Ohm}$ resistor between the power supply and the grid (this resistor should be left outside the chamber).

4. Place a switches in between the igniter supply and the keeper circuit. Connect the igniter supply to the discharge chamber according to fig D.7. Ensure that the $100 \mathrm{Ohm}$ resistor is placed outside the chamber.

5. Using alligator clips, banana cables, and bnc to banana connectors, connect the CTFT neutralizer to its power supply according to the circuit diagram in fig D.7.

6. Using kapton tape, attach a thermocouple to MiXI's discharge chamber. 
7. Carefully replace the bell jar. DO NOT allow the bell jar to come into contact with any of the test equipment. If necessary, apply high-vacuum grease to the jar's O-rings prior to this step.

8. Perform connectivity test. Use a multimeter in connectivity mode to check the connection between the keeper terminal, heater terminal, and the cathode chassis. The multimeter should indicate connectivity between the keeper terminal and keeper electrode and isolation from the cathode chassis and the heater terminal. The multimeter should indicate that the heater electrode IS electrically connected to the cathode's chassis and the ground wire. MiXI must be completely isolated from the hollow cathode

9. Use zip-ties or kapton tape to secure any loose ends of cables and/or thermocouples. If cables or wires are dangling in the bottom half of the chamber, they can fall into the gap between the gate valve and the cryo chamber once the gate valve is open. This makes completely closing the gate vale impossible.

Note: Should a loose end of a wire prevent the gate valve from closing all the way, DO NOT attempt to re pressurize and open the vacuum chamber. Turn off the compressor and wait (3-4 hours) until the cryo chamber temperature has reached room temperature. Introducing air into the cryo chamber while it is cold can damage the cryo.

10. Once the connectivity check has been performed and loose ends of wires are secured, lower the hoist to close the vacuum chamber and proceed to the Propellant System Setup Procedure. 


\section{D.3.4 Propellant System Setup Procedure}

The propellant system for MiXI is identical to that of the Hollow Cathode. Please refer to the Hollow Cathode Propellant System Setup Procedure.

\section{D.3.5 MiXI Operating Procedure}

1. (This is the most important step of the entire procedure.) While the chamber is pumping down, open all the valves in the propellant feed line up to (but not including) the propellant tank. Ensure that the diaphragm valve (if necessary), the needle valve, and the valve on the regulator are completely open. Turn the big knob on the Smith regulator in the direction of the Increase arrow to open the regulator. The downstream gague on the regulator should drop to the lowest reading (-30 psi).

Note: It is extremely important that there is absolutely NO AIR in the propellant feed line between the xenon tank and the cathode. If air is present in the system when the cathode is hot, the cathode insert will be poisoned.

2. Once the proper base pressure ( $\leq 5.0 e^{-6}$ Torr) has been reached, turn on the heater power supply. Set it to current controlled, 6A. Let the cathode heat up for about 15-20 minutes. Do not exceed 6.05A of current.

3. Open the propellant tank. The regulator should jump from -30 psi to 20-25 psi.

4. Close the needle valve until the flow meter reads at least 3.5 SCCM. 
Note: This flow rate includes the nitrogen-to-xenon conversion factor. The flow meter is calibrated for nitrogen so a conversion factor of 1.44 must be applied in order to determine the actual xenon flow rate.

5. Turn on the keeper power supply. It should automatically be set to voltage control. Increase the voltage until the keeper strikes. (You will know when this happens when the current indicated by the power supply abruptly spikes and the power supply switches to current control. Also, you will notice a purple glow emanating from the cathode.) Striking the cathode can theoretically occur around $120 \mathrm{~V}$, but in practice, the keeper may require as much as $1 \mathrm{kV}$ to strike. If the keeper does not strike at $120 \mathrm{~V}$, continue to increase the keeper voltage until it strikes or the maximum voltage of the power supply has been reached (300V for the Agilent or Sorinson supplies in lab). If the keeper strikes, you will not need the Glasman power supply and you may skip to step 8 .

Note: If the keeper supply is consistently drawing current before the voltage reaches about $50 \mathrm{~V}$, there may be a short between the keeper and the heater. Should this happen, turn everything off, wait for the cathode to cool, and perform a connectivity check on the cathode.

6. If the maximum voltage on the keeper's power supply has been reached, and the cathode has yet to ignite, then you will need to use the igniter supply (Glasman high voltage power supply). Ensure that the current and voltage knobs on the Glasman are set to zero before turning on the power supply. With the keeper power supply still on, switch on the Glasman and increase the current to $0.125 \mathrm{~A}$ (the maximum for this supply). Slowly increase the voltage on the Glasman until the keeper strikes. If the Glasman voltage is 
increased to $1 \mathrm{kV}$ without ignition, turn off the keeper and igniter supplies and return to step 5 .

7. Once the cathode has ignited, adjust the current on the keeper supply to the desired operational range (1-3A).

Note: The keeper power supply my oscillate between current control and voltage control as the cathode sputters and attempts to ignite. When this is happening, increase the current on the keeper supply until the current becomes steady (usually between 1-3 Amperes). Do not adjust or shut down the Glasman power supply until the cathode current is running steadily.

8. Turn off the igniter (Glasman) power supply.

9. Turn off the heater power supply.

10. Turn on the discharge (anode) power supply. Adjust the current and voltage until the desired discharge current has been reached.

11. Slowly close the needle valve until the desired propellant flow rate is reached.

12. Switch over the igniter power supply to the screen. Set the screen to the desired voltage and current (typically 750V and 125 milliAmps).

13. Turn on the accel power supply and set the desired voltage (typically 100V).

14. Allow the MiXI to operate steadily until the thermocouple indicates that the temperature at the magnets is greater than $280^{\circ} \mathrm{C}$ (typically 5-9 minutes).

Note: The SmCo magnets may be permanently damaged if their temperature ever exceeds $300^{\circ} \mathrm{C}$. 
15. Turn off the power supplies in reverse order of when they were turned on.

16. Close the diaphragm and/or needle valve to stop propellant flow.

17. Allow the cathode to cool for a minimum of two hours before opening the chamber. Once all tests are complete, follow the "Experimental Removal Procedure" to remove the apparatus from the chamber.

\section{D.3.6 Troubleshooting, Tips, and Tricks}

- Just FYI, after the screen and accel power supplies are on, arcing will occur between the grids and other metallic objects within the chamber. This may introduce error into any experimental data that is being collected.

- The heat from the hollow cathode's heater is transferred to MiXI. The longer the heater is left on prior to striking the keeper, the hotter MiXI will get; this affects the amount of time MiXI can be operated. 